\title{
Mapping the outer bulge with RRab stars from the VVV Survey
}

\author{
F. Gran ${ }^{1,2}$, D. Minniti ${ }^{3,2,4}$, R. K. Saito ${ }^{5}$, M. Zoccali ${ }^{1,2}$, O. A. Gonzalez ${ }^{6,7}$, C. Navarrete ${ }^{1,2}$, M. Catelan ${ }^{1,2}$, \\ R. Contreras Ramos ${ }^{2,1}$, F. Elorrieta ${ }^{8,2}$, S. Eyheramendy ${ }^{8,2}$, and A. Jordán ${ }^{1,2}$ \\ ${ }^{1}$ Instituto de Astrofísica, Pontificia Universidad Católica de Chile, Av. Vicuña Mackenna 4860, 782-0436 Macul, Santiago, Chile \\ e-mail: fgran@astro.puc.cl \\ 2 Millennium Institute of Astrophysics, Santiago, Chile \\ 3 Departamento de Ciencias Fisicas, Universidad Andres Bello, Republica 220, Santiago, Chile \\ 4 Vatican Observatory, 00120 Vatican City State, Italy \\ ${ }^{5}$ Universidade Federal de Sergipe, Departamento de Física, Av. Marechal Rondon s/n, 49100-000 São Cristóvão, SE, Brazil \\ ${ }^{6}$ European Southern Observatory, 3107 Alonso de Cordova, Vitacura, Santiago, Chile \\ 7 Institute for Astronomy, University of Edinburgh, Royal Observatory, Blackford Hill, Edinburgh, EH9 3HJ, UK \\ ${ }^{8}$ Departmento de Estadística, Facultad de Matemáticas, Pontificia Universidad Católica de Chile, Av. Vicuña Mackenna 4860, \\ 7820436 Macul, Santiago, Chile
}

Received 6 October 2015 / Accepted 4 April 2016

\begin{abstract}
Context. The VISTA Variables in the Vía Láctea (VVV) is a near-IR time-domain survey of the Galactic bulge and southern plane. One of the main goals of this survey is to reveal the 3D structure of the Milky Way through their variable stars. In particular, enormous numbers of RR Lyrae stars have been discovered in the inner regions of the bulge $\left(-8^{\circ} \lesssim b \lesssim-1^{\circ}\right)$ by optical surveys such as OGLE and MACHO, but leaving an unexplored window of more than $\sim 47$ sq deg $\left(-10.0^{\circ} \lesssim \ell \lesssim+10.7^{\circ}\right.$ and $\left.-10.3^{\circ} \lesssim b \lesssim-8.0^{\circ}\right)$ observed by the VVV Survey.

Aims. Our goal is to characterize the RR Lyrae stars in the outer bulge in terms of their periods, amplitudes, Fourier coefficients, and distances in order to evaluate the 3D structure of the bulge in this area. The distance distribution of RR Lyrae stars will be compared to that of red clump stars, which is known to trace a X-shaped structure, in order to determine whether these two different stellar populations share the same Galactic distribution.

Methods. A search for RR Lyrae stars was performed in more than $\sim 47 \mathrm{sq} \mathrm{deg}$ at low Galactic latitudes $\left(-10.3^{\circ} \lesssim b \lesssim-8.0^{\circ}\right)$. In the procedure the $\chi^{2}$ value and analysis of variance (AoV) statistic methods were used to determine the variability and periodic features of the light curves, respectively. To prevent misclassifications, the analysis was performed only on the fundamental mode RR Lyrae stars (RRab) owing to similarities found in the near-IR light curve shapes of contact eclipsing binaries (W UMa) and first overtone RR Lyrae stars (RRc). On the other hand, the red clump stars of the same analyzed tiles were selected, and cuts in the color-magnitude diagram were applied and the maximum distance restricted to $\sim 20 \mathrm{kpc}$ in order to construct a similar catalog in terms of distances and covered area compared to the RR Lyrae stars.

Results. We report the detection of more than 1000 RR Lyrae ab-type stars in the VVV Survey located in the outskirts of the Galactic bulge. A few of them are possibly associated with the Sagittarius Dwarf Spheroidal Galaxy. We calculated colours, reddening, extinction, and distances of the detected RR Lyrae stars in order to determine the outer bulge 3D structure. Our main result is that, at the low galactic latitudes mapped here, the RR Lyrae stars trace a centrally concentrated spheroidal distribution. This is a noticeably different spatial distribution to the one traced by red clump stars known to follow a bar and X-shaped structure. We estimate the completeness of our sample at $80 \%$ for $K_{\mathrm{s}} \leq 15 \mathrm{mag}$.
\end{abstract}

Key words. Galaxy: bulge - Galaxy: stellar content - Galaxy: structure - infrared: stars - surveys - stars: variables: RR Lyrae

\section{Introduction}

Big astronomical surveys are changing the way we understand the formation, structure and evolution of our Galaxy. Among these surveys, only a few have been able to access the inner regions of the Milky Way because of the effects of severe crowding and high interstellar extinction of these dense Galactic regions. Near- and mid-IR surveys such as 2MASS, GLIMPSE, and UKIDSS-GPS (Skrutskie et al. 2006; Benjamin et al. 2005; Lucas et al. 2008) have helped to overcome the extinction problem covering the innermost regions of the Galaxy, but the lack of multiple-epoch observations within those surveys prevents us from using them to study and characterize the large number of variable sources in the bulge. Optical time-domain surveys such as OGLE, MACHO, and EROS (Udalski et al. 2015; Alcock et al. 1996; Aubourg et al. 1993) have partially solved this problem but unfortunately the high extinction found towards the bulge line of sight restricts them from accurately mapping the innermost regions.

In response to these limitations, the VISTA Variables in the Vía Láctea (VVV) ESO public survey (Minniti et al. 2010) provides near-IR, multi-epoch photometric coverage of the inner Galaxy $\left(-10^{\circ} \lesssim \ell \lesssim 10^{\circ},-10^{\circ} \lesssim b \lesssim 5^{\circ}\right)$. The large near-IR coverage of the VVV survey, high spatial resolution, and depth of the survey enables comprehensive studies across the entire inner Galaxy, reaching larger distances than has ever been possible. The first stage of the VVV Survey provided full-coverage, multicolour photometry of the inner $520 \mathrm{sq}$ deg of the Galaxy. These data were used for the construction of 2D and 3D extinction maps (Gonzalez et al. 2011, 2013; Schultheis et al. 2014), and metallicity gradient maps (Gonzalez et al. 2013) of the Galactic bulge.

One of the main scientific goals of the VVV Survey is to build a comprehensive 3D map of the Milky Way using 
well-known primary distance indicators. In this context, the first epoch of VVV observations has been used to investigate the shape of the bulge using the observed magnitude of red clump giant stars as distance indicators. Bulge studies using red clump (RC) stars have helped to unveil the overall shape of the stellar bar, confirming that the Milky Way hosts a peanut- or X-shape bulge (Wegg \& Gerhard 2013; Saito et al. 2012b).

On the other hand, the ongoing variability campaign of the VVV survey now allows us to investigate the shape of the inner Galaxy using variable stars as distance estimators. Variable star searches are expected to yield many more candidates in the near future (Catelan et al. 2013a,b), allowing us to measure the extinctions and distances along the line of sight, providing another 3D view of the inner Milky Way (Dékány et al. 2013, 2015). RR Lyrae stars are particularly interesting in this context as they allow us to unequivocally trace the oldest stellar component of the Galaxy (Dékány et al. 2013; Catelan \& Smith 2015). Interestingly, the distance distribution of RR Lyrae stars found by Dékány et al. (2013) follows a different shape than that traced by red clump stars. While the distances obtained from red clump stars trace closely the position angle of the bar and also the distance split along the minor axis due to the far and near arms of the X-shaped bulge, distances and radial velocities to the RR Lyrae population from Dékány et al. (2013) and Kunder et al. (2016), respectively, appear to follow a spheroidal distribution instead of the stellar bar traced by red clump stars.

In the present study we perform the search of RR Lyrae stars using VVV data and continue the analysis started by Gran et al. (2015), extending the work to 28 more VVV tiles (b201-b228). These regions have been not been covered by the OGLE survey yet; therefore, the RR Lyrae stars presented here are particularly important in this context. This is where the X-shaped bulge becomes most prominent, making it the ideal location to investigate how different the structures traced by these two populations are. We calculated their distances and compared their spatial distribution with respect to those derived from red clump stars.

\section{Observations}

The VVV Survey is a public ESO near-IR survey that is mapping the inner Milky Way, including the inner halo, the bulge and an adjacent section of the disk with the VISTA $4 \mathrm{~m}$ telescope at the ESO Paranal Observatory (Minniti et al. 2010). The survey covers a total area of $562 \mathrm{sq} \mathrm{deg}$; and the VVV database now contains $Z Y J H K_{\mathrm{s}}$ photometry of about one billion sources on the VISTA system for which 2MASS coordinates have been used to construct the coordinate system, and a variability campaign in the $K_{\mathrm{s}}$-band (Saito et al. 2012a; Hempel et al. 2014). See Gran et al. (2015) for more details on the instrument and their spatial configuration of the Galactic bulge and disk.

In this analysis we used data covering more than $\sim 47 \mathrm{sq} \mathrm{deg}$ in the outer bulge $\left(-10.0^{\circ} \lesssim \ell \lesssim+10.7^{\circ}\right.$ and $-10.3^{\circ} \lesssim$ $\left.b \lesssim-8.0^{\circ}\right)$. This area corresponds to the VVV tiles $b 201$ through b228, obtained between April 2010 and August 2014 with 60-62 epochs in all the selected tiles. We use aperture photometry applied to the stacked images known as tiles, provided by the Cambridge Astronomical Survey Unit (CASU) ${ }^{1}$ and setting the minimum number of epochs per star analyzed to 30 in order to achieve a better frequency analysis and avoid gaps in the light curves.

1 http://casu.ast.cam.ac.uk/vistasp/

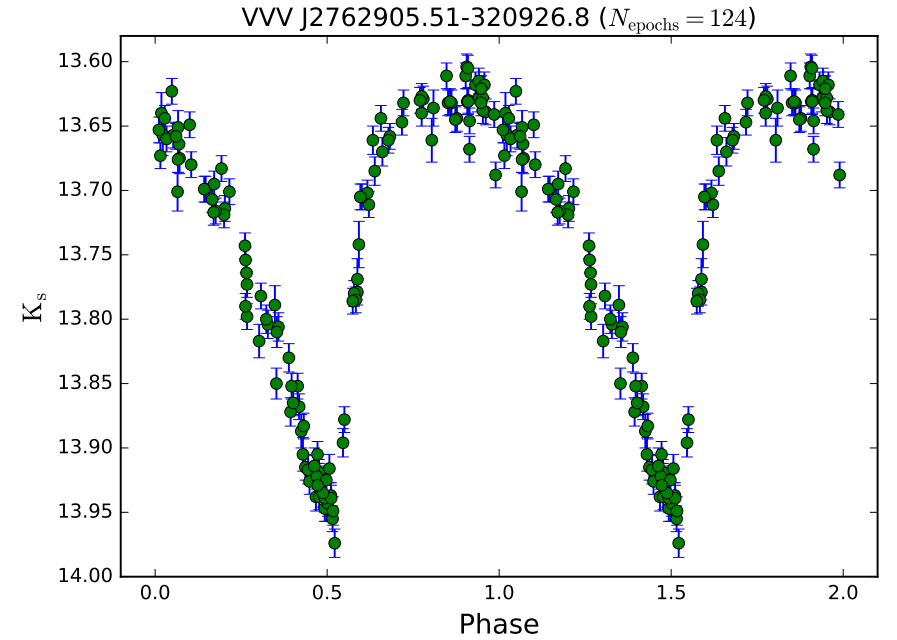

Fig. 1. RR Lyrae star in the overlap of two adjacent tiles (b208 and b222). The light curve has the maximum number of epochs in our sample $(62 \times 2=124)$.

\subsection{Detection and classification of $R R$ Lyrae stars}

We selected variable candidates by analyzing the $\chi^{2}$ value for all the available time series, considering the mean error-weighted magnitude as the model (e.g. a non-variable star will have values close to 0). A similar analysis was presented in Carpenter et al. (2001) to detect variable candidates. If this value exceeds the imposed cutoff of $\chi^{2}=2$ (see Gran et al. 2015), the time-series periodicities are tested by the analysis of variance (AoV) statistic (Schwarzenberg-Czerny 1989) in the RR Lyrae stars period range $(0.2 \leq P$ (days) $\leq 1.2)$. After this process the light curves were visually classified.

We repeated the classification process over the 28 analyzed tiles (b201-b228) and checked whether there were duplicates in our catalogs. RR Lyrae stars in the intersection areas are also important in order to check the parameters derived from two independent light curves. The tiling pattern produces overlapping areas of about $7 \%$ between the tiles; Saito et al. (2012b) thus took advantage of the duplicated RR Lyrae stars in the overlapping regions by combining their data. Figure 1 shows a RRab star with the maximum number of epochs found in the intersection between the VVV tiles $b 208$ and $b 222$. For the overlapping RR Lyrae light curves, the derived periods, amplitudes, and mean magnitudes were compared, and resulted in a distribution of the parameters that was close to zero within the errors.

In this process we assign a label to the RRab stars according to their narrow period range $(\sim 0.4 \leq P$ (days) $\leq 1.2)$, near-IR amplitude $\left(0.2 \lesssim A_{\mathrm{K}_{\mathrm{s}}}(\mathrm{mag}) \lesssim 0.5\right)$, and characteristic asymmetric light curve shape (see Fig. 1). As reported by Alonso-García et al. (2015), in the near-IR bands there are fewer features that can be used to classify different variable types than in the optical regime. Therefore, because the light curves of the RRc stars in the near-IR mimic the behaviour of other variable classes such as W UMa contact binaries and longperiod SX Phe pulsating variables, likely RRc stars $(P$ (days) $\lesssim$ $0.4)$ are not under analysis here. In addition to the human expert classification described above, we ran the light curves through a machine-learning classifier specifically developed for the classification of RRab in the VVV Survey. The classifier is based on a set of features extracted from each light curve following a similar approach to that of Debosscher et al. (2007) and Richards et al. (2011), and will be described elsewhere 


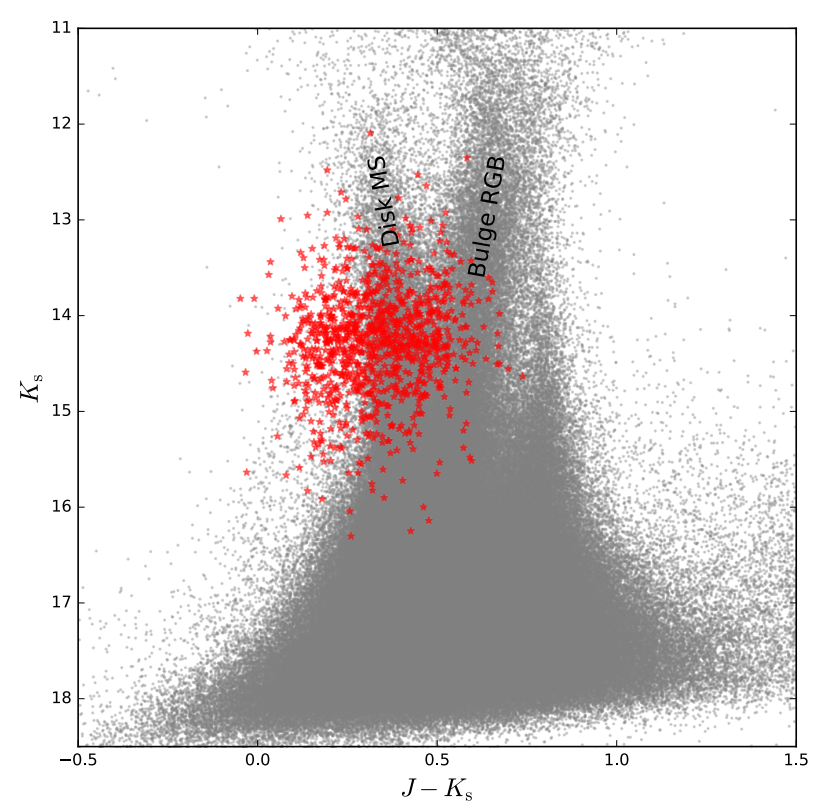

Fig. 2. $K_{\mathrm{s}}$ vs. $\left(J-K_{\mathrm{s}}\right) \mathrm{CMD}$ of the complete catalog of RR Lyrae stars (red stars) compared with the sources in tile $b 201$ as background. The CMD shows two prominent features, the disk main sequence (MS) and the bulge red giant branch (RGB), which are identified in the figure.

(Elorrieta et al., in prep.). We will use a similar classifier in the near future to produce a catalog of VVV variable sources classified using automated procedures (for more details see Catelan et al. 2013b; Angeloni et al. 2014).

One of the 28 tiles explored is obliterated by the presence of a very bright star, resulting in fewer RR Lyrae discovered. Tile b205 contains the star $\eta$ Sgr (HD 167600), which is very bright in the near-IR with $K_{\mathrm{s}} \sim-1.55 \mathrm{mag}$. Such a bright star not only saturates the detector, but also causes reflections that affect the flat fields; the resulting mosaic of this tile contains regions that are not suitable for variability searches. This is the reason why tile $b 205$ contains fewer RRab stars $\left(N_{\mathrm{RRab}}=31\right)$ than the rest of the tiles $\left(N_{\mathrm{RRab}} \sim 37\right.$ on average $)$.

Our RRab light curves have 60-62 data points with a median magnitude of $K_{\mathrm{s}}=14.2 \mathrm{mag}\left(12.1 \lesssim K_{\mathrm{s}} \lesssim 16.3\right)$. At this magnitude level the completeness of the VVV source catalogues is high, with about $95 \%$ detection efficiency in less crowded fields such as the outermost bulge region (Saito et al. 2012a). On the other hand, experiments of signal detection rates based on VVV data for RRab stars reach about $90 \%$ detection when applied to light curves with 60 epochs (Catelan et al. 2013b). Therefore, we can estimate the completeness of our RRab sample as accurately as $80 \%$ for $K_{\mathrm{s}} \lesssim 15 \mathrm{mag}$, with no expected trends along the two axes, since crowding and extinction are similar across the analyzed area. At fainter magnitudes the completeness is smaller and makes it difficult to find the most distant RR Lyrae, for example the ones that may belong to the Sgr dwarf galaxy. However, we identify a few Sgr RR Lyrae candidates (see Sect. 3.1).

We also checked the completeness of our catalogue by comparing our findings with the RR Lyrae found by OGLE in a small fraction of our area which overlaps an OGLE IV field (Soszyński et al. 2014). There are 22 RR Lyrae stars with $-10.3^{\circ} \lesssim b \lesssim-8.0^{\circ}$ in the OGLE IV catalog, of which we will only focus on the $13 \mathrm{RRab}$ stars present. In our catalog there are eight matches within $d<1^{\prime \prime}$ in tiles $b 220$ and $b 221$. Three of the five remaining RRab stars were not analyzed by our algorithm owing to non-stellar photometry flags or fewer epochs than the minimum required, and there were no matches at $1^{\prime \prime}$ for
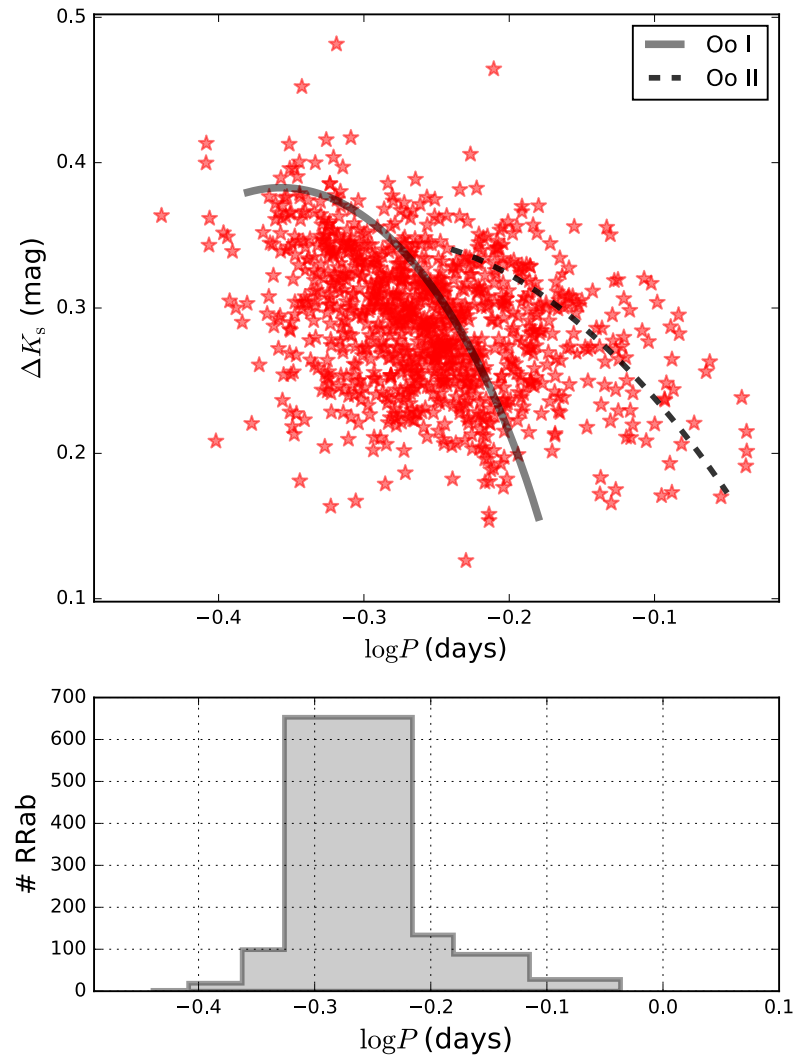

Fig. 3. Top panel: Bailey diagram of the complete RRab catalog. The OoI (solid) and OoII (dashed) lines derived by Navarrete et al. (2015) are shown. Bottom panel: period histogram of the 1019 RRab stars with bins adapted by the Bayesian Block algorithm (Scargle et al. 2013) through the astroML implementation (Vanderplas et al. 2012).

the last two RRab stars in the area in our catalog. With these corrections our completeness with respect to the OGLE survey is at least $80 \%$. Certainly, not all of the RRab stars in the catalog are new discoveries. We match our catalog with the General Catalogue of Variable Stars (GCVS; Samus et al. 2009) and find a total of 207 matches. VVV IDs and the respective GCVS names for matching objects are presented in Appendix A. We note that none of our classified RRab stars has tagged eclipsing binary counterparts in the GCVS, even though we do not discard minor contamination due to eclipsing binaries that can mimic RRab stars. Finally, 27 of the RRab stars in the tile $b 201$ have already een reported y Gran et al. (2015).

\section{Results}

After accounting for the duplicates, a total of 1019 RRab stars remained in our sample. The final catalogue is presented in Appendix A. In the first step we characterized this sample in terms of its calculated magnitude-weighted $\left\langle K_{\mathrm{s}}\right\rangle,\langle J\rangle-\left\langle K_{\mathrm{s}}\right\rangle$ colour, periods, amplitudes, light curve shapes, and, coordinates. Figure 2 shows the $J-K_{\mathrm{s}}$ colour-magnitude diagram (CMD) for the complete RR Lyrae catalog with tile $b 201$ as a comparison field. The RR Lyrae stars lie in a wide range of mean- $K_{\mathrm{s}}$ magnitudes owing to their distance distribution in the Galaxy, but the $J-K_{\mathrm{s}}$ colour is limited between $\sim 0.0$ and 0.6 , similar values to those reported by Gran et al. (2015).

In addition to the locus on the CMD, the RR Lyrae stars can be identified by their position on the Bailey diagram (Bailey 1902), which relates the amplitude of the RR Lyrae stars with the period distribution of the entire sample (Fig. 3). In this 

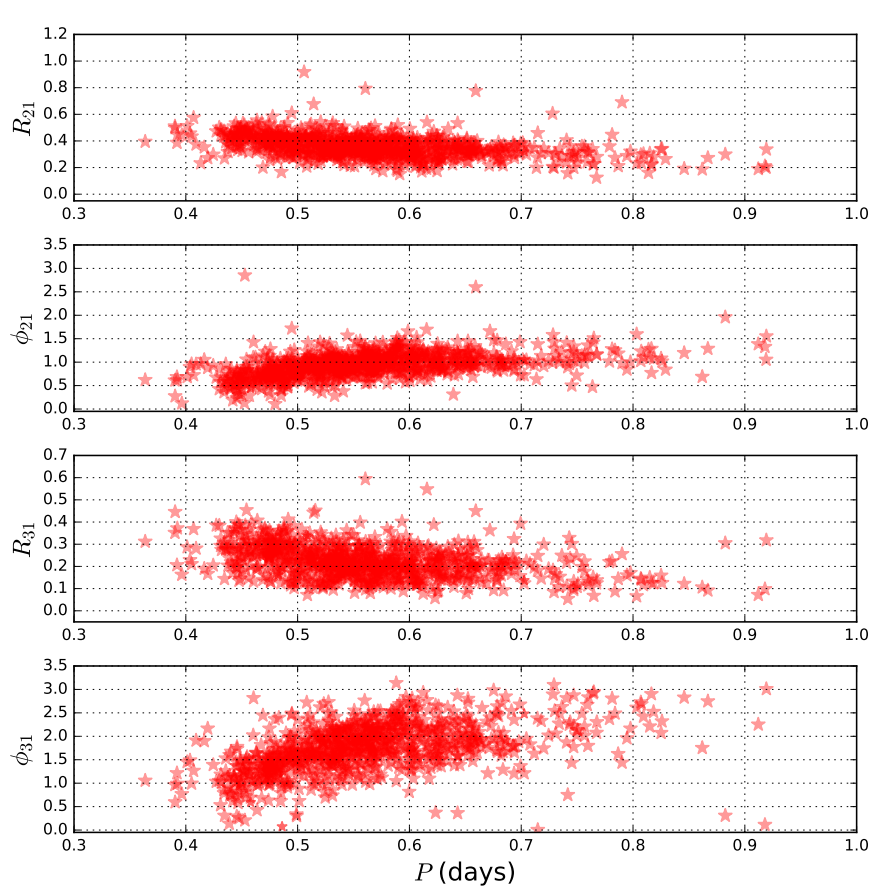

Fig. 4. Top to bottom: $R_{21}, \phi_{21}, R_{31}$, and $\phi_{31}$ coefficients of a Fourier series (sine based) using the DFF routine.

diagram we can see that our RR Lyrae stars are predominantly Oosterhoff Type I (OoI) with a minor composition of Oosterhoff Type II (OoII). We derived this composition with the Oosterhoof reference lines traced by Navarrete et al. (2015).

In addition to the period and amplitude, another characteristic feature of the RRab stars is the light curve shape, which can be described by a Fourier series. A sine decomposition up to sixth order was performed with the direct Fourier fitting (DFF) routine given by Kovács \& Kupi (2007). Figure 4 shows the $R_{21}, \phi_{21}, R_{31}$, and $\phi_{31}$ coefficients as function of the period. All the Fourier components tend to be clustered in a limited region in this space (for reference see Fig. 6 of Deb \& Singh 2010). There were some outliers in the distributions (e.g.: RRab with $R_{21}>0.6$ or $\phi_{21}>2$ ), which were visually inspected; some gaps in the light curve were found that have an effect on the final value.

The spatial distribution in Galactic coordinates of the cata$\log$ is shown in Fig. 5. The observations span only $2^{\circ}$ in $b$, but more than $20^{\circ}$ in $\ell$, resulting in the very elongated shape of the figure. Although there are no globular clusters in the analyzed area according to the Francis \& Anderson (2014) catalog, their presence in nearby regions could bias the number of RR Lyrae stars found. This possible effect on our catalog was investigated on the three closest globular clusters to our sample of RRab stars. NGC 6656 is the only cluster that has associated RR Lyrae stars according to Clement et al. (2001, 2015 editiononline catalog ${ }^{2}$ ), but the closest variable is $10^{\prime}$ further from the cluster tidal radius $\left(r_{t} \approx 30^{\prime}\right)$ given by the 2010 version of the Harris (1996) catalog. NGC 6624 and 6637 are considered metal-rich clusters with $[\mathrm{Fe} / \mathrm{H}]$ values of -0.63 and -0.77 , respectively (Valenti et al. 2004, 2005). Both clusters develop a very red horizontal branch, which is the reason why they are not known to have associated RR Lyrae stars.

2 http://www. astro.utoronto.ca/ cclement/read.html

\subsection{Distances and the $3 D$ view of the outer bulge}

One of the main goals of the VVV Survey is to trace the Galactic structure using variable stars in order to make the most complete 3D view of the central regions of our Galaxy (Minniti et al. 2010). The primary distance indicators are the RR Lyrae stars owing to the high number density present in the bulge area (Soszyński et al. 2014) and the tight period-luminosity (P-L) relation that they follow in near-IR bands (Longmore et al. 1990; Catelan et al. 2004). To obtain the distance values, first we must calculate the reddening and extinction values to the individual variables. The former can be obtained through the difference between the mean-apparent and absolute magnitudes of our RRab stars, given by

$E\left(J-K_{\mathrm{s}}\right)=\left(J-K_{\mathrm{s}}\right)-\left(J-K_{\mathrm{s}}\right)_{0}=\left(J-K_{\mathrm{s}}\right)-\left(M_{J}-M_{K_{\mathrm{s}}}\right)$,

where $\left(J-K_{\mathrm{s}}\right)_{0}$ is the intrinsic colour of our RRab star and $M_{X}$ the absolute magnitude in the $X$-band. In our analysis we adopt the P-L relations derived by Alonso-García et al. (2015) to recover the absolute magnitudes of the RR Lyrae stars in the $J$ - and $K_{\mathrm{s}}$-bands with $\log Z=[\mathrm{Fe} / \mathrm{H}]-1.765$, based on a solar metallicity of $Z_{\odot}=0.017$ (Catelan et al. 2004). To calculate the $J$-band mean magnitudes for the stars in our catalog we performed a linear regression between the $J$ - and $K_{\mathrm{s}}$-band mean magnitudes of the RRab stars of $\omega$ Centauri studied by Navarrete et al. (2016). This analysis is needed because the VVV Survey only provides one observation in the $Z Y J H$-bands. The resulting fit is given by $\langle J\rangle=0.93 \times\left\langle K_{\mathrm{s}}\right\rangle+1.26$. As expected, the residuals are centred in 0 with a dispersion of 0.03 mag. This allows us to derive the reddening on a star-by-star basis, and also the extinction of each RRab star by adopting an extinction law (e.g. Cardelli et al. 1989).

At this point we calculate the distances given by

$\log d=1+0.2\left(K_{\mathrm{s}, 0}-M_{K_{\mathrm{s}}}\right)$,

with $d$ the individual distance in pe to our RRab stars. Figure 6 shows the distribution of distances of the RRab stars in our cata$\log$. The vertical line corresponds to the Galactic centre distance derived in Dékány et al. (2013) with a value of $R_{0} \approx 8.33 \mathrm{kpc}$. Our distances have a maximum frequency around $R_{0}$ where the centre of the distribution is, and an asymmetric shape towards the far side of the bulge because the volume observed is greater owing to the cone effect. According to their distances, some of the RR Lyrae stars may belong to the Sagittarius dwarf spheroidal (Sgr dSph) galaxy (e.g. distances around $20 \mathrm{kpc}$ ). Kunder \& Chaboyer (2009) place the core of the Sgr dSph galaxy at $\sim 22-27 \mathrm{kpc}$ from the Sun, but $\sim 4^{\circ}$ away from our analyzed region. Even taking into account that Sgr RR Lyrae stars are mixed with the Milky Way halo variables, some RR Lyrae stars found towards these coordinates have been associated with the dwarf galaxy by MACHO (Alard 1996; Alcock et al. 1997) and OGLE (Soszyński et al. 2014).

The elongated shape of the analyzed area allows us to approximate the observation volume with a circular sector, projecting the $b$ coordinate. Figure 7 shows distances and Galactic longitude in this line-of-sight circular projection. The RR Lyrae stars tend to stay near the projected Galactic centre distance $(d \approx 8 \mathrm{kpc})$ and the previously mentioned Sgr dSph RR Lyrae candidates are clearly visible in the $16 \leq d(\mathrm{kpc}) \leq 22$ and $\ell \geq 6^{\circ}$ zone.

\subsection{To trace or not to trace: the $X$-shaped problem}

Many efforts have been made to study the 3D structure of the Milky Way through its stellar content. Pulsating variable stars 


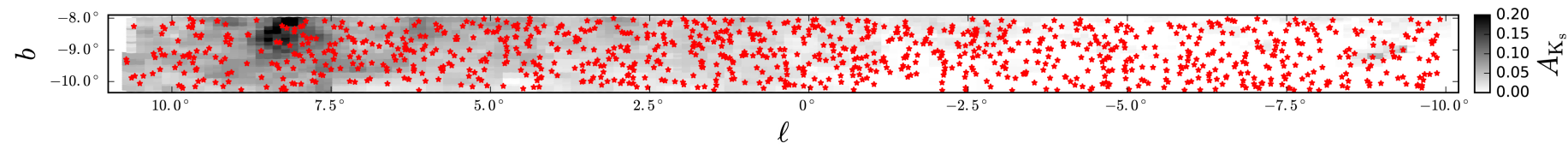

Fig. 5. Spatial distribution in Galactic coordinates $(\ell, b)$ of the RRab stars found in this work.

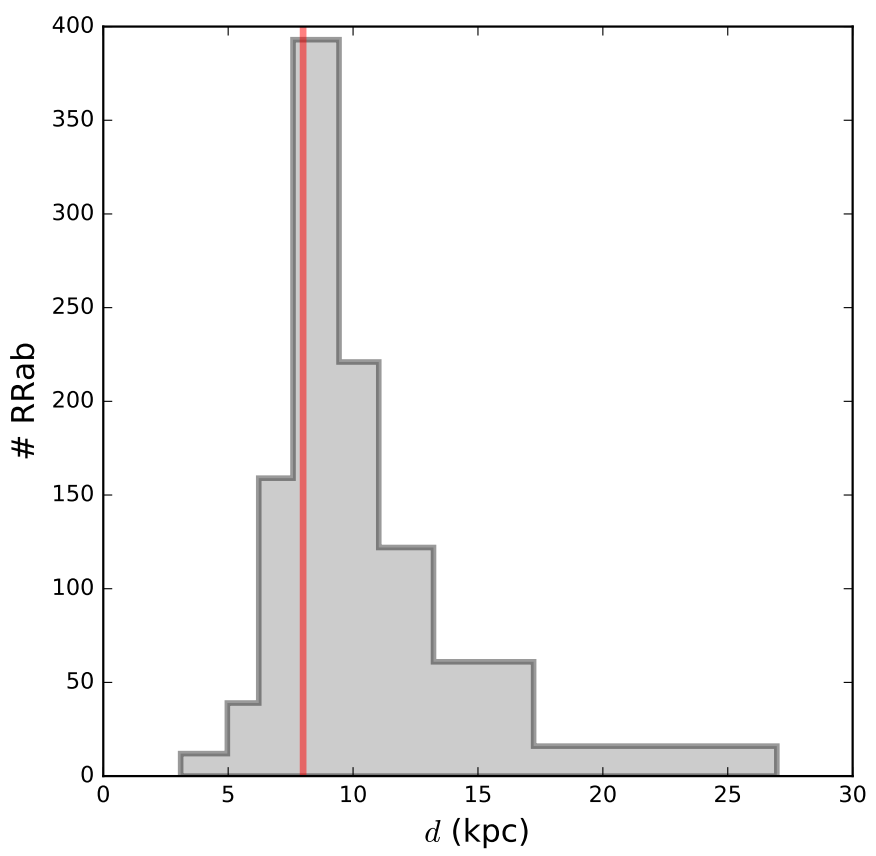

Fig. 6. Distribution of distances of the RR Lyrae stars found. The vertical line represents the Galactic centre derived by Dékány et al. (2013) with OGLE-III RR Lyrae stars of $R_{0} \approx 8.33 \mathrm{kpc}$.

are important distance indicators (e.g. RR Lyrae and Cepheids, among others), but in addition to this method, the red clump stars were also used in near-IR single-epoch studies to derive accurate distances to the Milky Way edge (Minniti et al. 2011), bulge (Alves 2000), or the Large Magellanic Cloud (Alves et al. 2002). This feature of the red clump stars has been used recently to discover the X-shaped structure of the Milky Way (McWilliam \& Zoccali 2010; Nataf et al. 2010; Saito et al. 2011; Wegg \& Gerhard 2013) that contains a bar at its central (Rattenbury et al. 2007; Gonzalez et al. 2011). This structure probably vanishes with decreasing metallicity of stars, and it is not expected in an old stellar population (Ness et al. 2012). It is clear and well studied that the red clump stars follow this barred Galactic feature, but in the RR Lyrae case there is no clear evidence for the same trend. On the one hand Pietrukowicz et al. (2012) with OGLE-III RR Lyrae stars claim the existence of the barred structure rotated about $30^{\circ}$ with respect to the line of sight between the Sun and the Galactic centre. On the other hand, Dékány et al. (2013) completely rule out this possibility using the same dataset, but included the near-IR results of the VVV Survey.

We used our catalogue to compare the distribution of RR Lyrae at low Galactic latitude with the distribution of red clump stars in the same analyzed tiles. Both catalogues were divided into three longitude bins: $-10^{\circ}<\ell<-3.5^{\circ} ;-3.5^{\circ}<\ell<$ $3.5^{\circ}$; and $3.5^{\circ}<\ell<10^{\circ}$. The red clump stars were selected with the same technique described in Minniti et al. (2011) with magnitudes $K_{\mathrm{s}}<15$, effectively limiting our study to red clump stars at distances closer than $\sim 20 \mathrm{kpc}$. The distributions of the red clump stars also include the contribution of the underlying RGB. The RGB does not change the position of the red clump, thus the distributions are suitable for our comparison purposes. Assuming an intrinsic red clump absolute magnitude $M_{K_{\mathrm{s}}}=-1.55$ and an intrinsic red clump colour $\left(J-K_{\mathrm{s}}\right)_{0}=0.68$, as given by Gonzalez et al. (2011) for Baade's window red clump stars, the distance equation yields

$\mu=-5+5 \log d(\mathrm{pc})=K_{\mathrm{s}}-0.73\left(J-K_{\mathrm{s}}\right)+2.05$,

where the Cardelli et al. (1989) extinction law was assumed.

Figure 8 shows the result of the comparison between the distance distribution of red clump and RR Lyrae stars. The red vertical line shows the mean distance value of a single-Gaussian fit to the RR Lyrae in each longitude bin, namely $d_{\text {RRL }} \sim 9.01$, 8.63 , and $8.98 \mathrm{kpc}$, from positive to negative longitudes, respectively, with associated standard deviations of $\sigma_{\mathrm{RRL}} \sim 1.36,1.31$, and 1.35. Clearly, the variation in the mean distance across the longitude direction is negligible for the RR Lyrae distribution. Red clump stars, on the contrary, show a single peak at $d_{\mathrm{RC}} \sim$ $6.8 \mathrm{kpc}$ at positive longitudes, two peaks at $d_{\mathrm{RC}} \sim 6.8$ and $9.5 \mathrm{kpc}$ across the minor axis, and a single peak at $d_{\mathrm{RC}} \sim 9.4 \mathrm{kpc}$ at negative longitudes. In all three cases, a two-sample KolmogorovSmirnov test reveals that the distributions of red clump and RR Lyrae stars are indeed different, with higher than $98.9 \%$ probability. This strongly suggests that the red clump stars (but not the RR Lyrae) follow the main Galactic bar, flaring up into a peanut shape (or X-shape) far away from the Galactic plane. The marked difference in the distance distribution of RR Lyrae variables and red clump stars confirms at low latitudes the conclusion by Dékány et al. (2013) that RR Lyrae and RC stars trace two different components in the bulge.

\section{Summary}

A search for RR Lyrae stars was performed in more than $\sim 47$ sq deg in the outer parts of the Galactic bulge observed by the VVV Survey. In total, more than 1000 fundamental mode RR Lyrae stars were found in this area, with an estimated completeness level of $80 \%$ for $K_{\mathrm{s}} \leq 15 \mathrm{mag}$. We analyzed their periods, amplitudes, light curve shapes, and 3D positions within the Galaxy. This sample allows us to compare the distribution along the Galactic longitude of RR Lyrae and red clump stars, resulting in statistically very significant differences of more than $1.5 \mathrm{kpc}$ between the peaks of both distributions. These differences prevail along the Galactic latitudes observed by the VVV Survey that shows an unchanged RR Lyrae distance distribution and a moving red clump distribution tracing the Milky Way bar. This result fully supports the work of Dékány et al. (2013) and Kunder et al. (2016), which postulates a spheroidal distribution of the RR Lyrae stars in the Galactic bulge, but does not trace the strong bar of the red clump stars. A complete view of the RR Lyrae stars over the entire Galactic bulge will be unveiled 


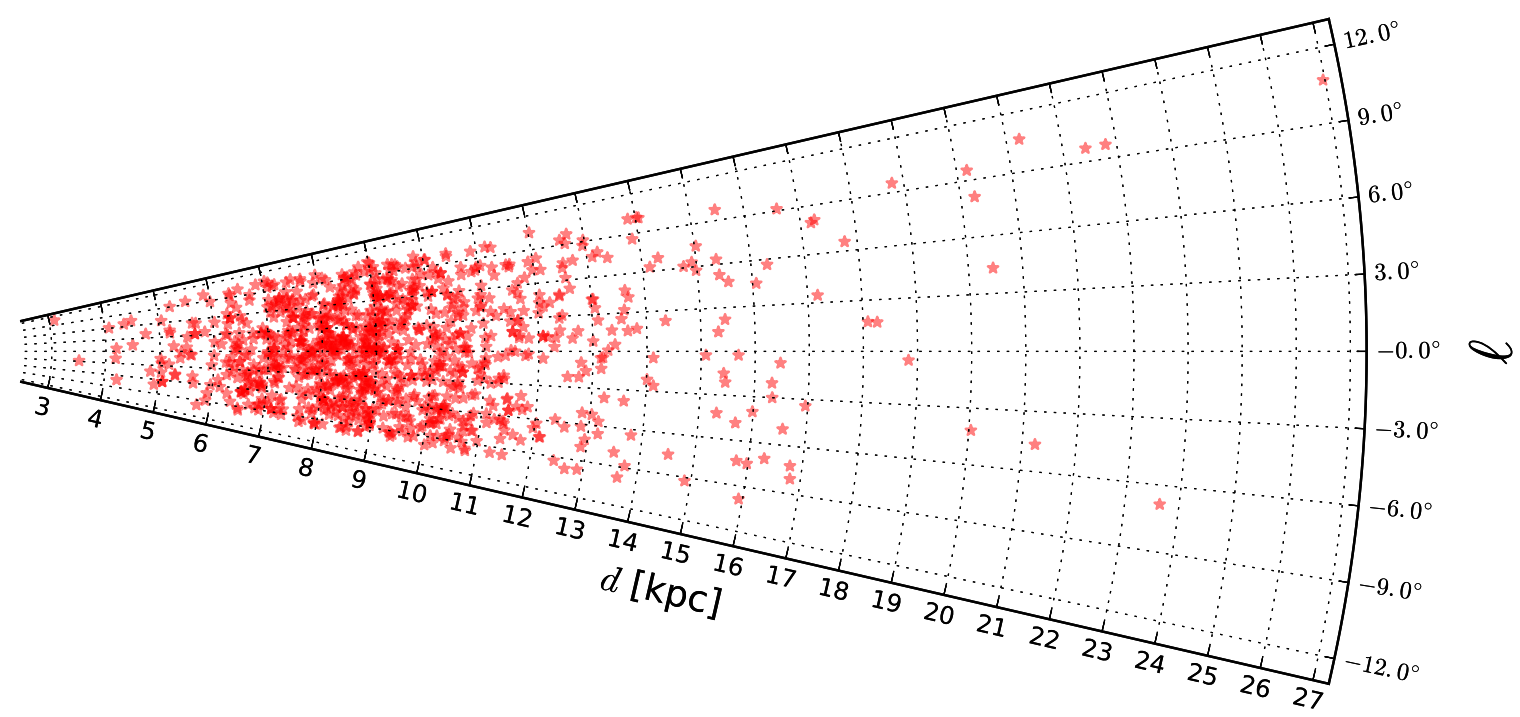

Fig. 7. Cone-view $(d, \ell)$ of the analyzed area in the Galactic bulge. The sample is concentrated around the projection of the Galactic centre.
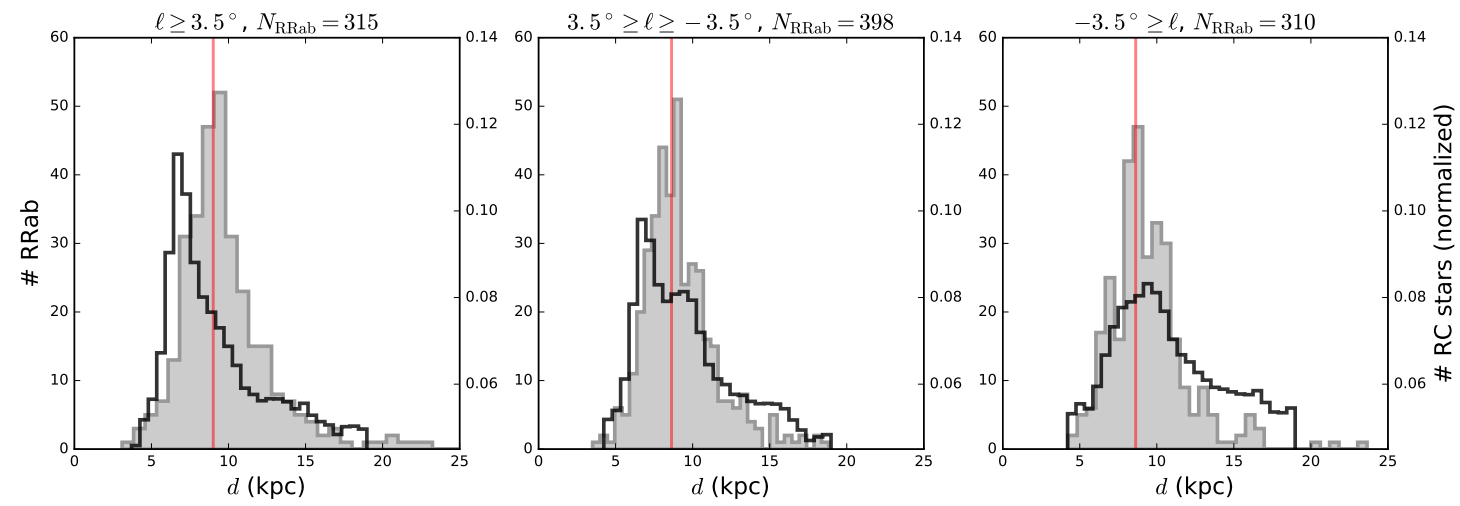

Fig. 8. Histogram of distances of RR Lyrae (filled) and red clump stars (steps) as function of Galactic latitude $(\ell)$. The distributions of the red clump stars include also the underlying RGB, but since those do not affect the position of the red clump the distributions are suitable for our comparison purposes. The total number of red clump stars in the same areas overwhelms the number of RR Lyrae, thus the histogram showing their distribution in distance was normalized for better visualization. The vertical line represents the RR Lyrae mean distance of each region.

when fully automatic searches in the VVV Survey area is completed (Catelan et al. 2013b; Angeloni et al. 2014).

Acknowledgements. We gratefully acknowledge the use of data from the ESO Public Survey program ID 179.B-2002 taken with the VISTA telescope and data products from the Cambridge Astronomical Survey Unit. Support for the authors is provided by the BASAL CATA Center for Astrophysics and Associated Technologies through grant PFB-06, and the Ministry for the Economy, Development, and Tourism's Programa Iniciativa Científica Milenio through grant IC120009, awarded to the Millennium Institute of Astrophysics (MAS). D.M. and M.Z. acknowledge support from FONDECYT Regular grants No. 1130196 and 1150345 , respectively. Partial support for this project is provided by CONICYT's PCI program through grant DPI20140066. F.G., C.N., and M.C. acknowledge support from FONDECYT regular grant No. 1141141. C.N. and F. G. acknowledge support from CONICYT-PCHA Doctorado and Magíster Nacional 2015-21151643 and 2014-22141509, respectively. R.K.S. acknowledges support from CNPq/Brazil through projects 310636/2013-2 and 481468/2013-7. We gratefully acknowledge the use of IPython, Astropy, AstroML, Matplotlib, TOPCAT, and ALADIN sky atlas.

\section{References}

Alard, C. 1996, ApJ, 458, L17

Alcock, C., Allsman, R. A., Axelrod, T. S., et al. 1996, AJ, 111, 1146

Alcock, C., Allsman, R. A., Alves, D. R., et al. 1997, ApJ, 474, 217

Alonso-García, J., Dékány, I., Catelan, M., et al. 2015, AJ, 149, 99

Alves, D. R. 2000, ApJ, 539, 732
Alves, D. R., Rejkuba, M., Minniti, D., \& Cook, K. H. 2002, ApJ, 573, L51 Angeloni, R., Contreras Ramos, R., Catelan, M., et al. 2014, A\&A, 567, A100 Aubourg, E., Bareyre, P., Brehin, S., et al. 1993, The Messenger, 72, 20

Bailey, S. I. 1902, Annals of Harvard College Observatory, 38,

Benjamin, R. A., Churchwell, E., Babler, B. L., et al. 2005, ApJ, 630, L149 Cardelli, J. A., Clayton, G. C., \& Mathis, J. S. 1989, ApJ, 345, 245

Carpenter, J. M., Hillenbrand, L. A., \& Skrutskie, M. F. 2001, AJ, 121, 3160

Catelan, M., \& Smith, H. A. 2015, Pulsating Stars (Wiley-VCH)

Catelan, M., Pritzl, B. J., \& Smith, H. A. 2004, ApJS, 154, 633

Catelan, M., Dekany, I., Hempel, M., \& Minniti, D. 2013a, Boletin de la Asociacion Argentina de Astronomia La Plata Argentina, 56, 153

Catelan, M., Minniti, D., Lucas, P. W., et al. 2013b, in 40 Years of Variable Stars: A Celebration of Contributions by Horace A. Smith, eds. K. Kinemuchi et al. [arXiv: 1310.1996]

Clement, C. M., Muzzin, A., Dufton, Q., et al. 2001, AJ, 122, 2587

Deb, S., \& Singh, H. P. 2010, MNRAS, 402, 691

Debosscher, J., Sarro, L. M., Aerts, C., et al. 2007, A\&A, 475, 1159

Dékány, I., Minniti, D., Catelan, M., et al. 2013, ApJ, 776, L19

Dékány, I., Minniti, D., Hajdu, G., et al. 2015, ApJ, 799, L11

Francis, C., \& Anderson, E. 2014, MNRAS, 441, 1105

Gonzalez, O. A., Rejkuba, M., Minniti, D., et al. 2011, A\&A, 534, L14

Gonzalez, O. A., Rejkuba, M., Zoccali, M., et al. 2013, A\&A, 552, A110

Gran, F., Minniti, D., Saito, R. K., et al. 2015, A\&A, 575, A114

Harris, W. E. 1996, AJ, 112, 1487

Hempel, M., Minniti, D., Dékány, I., et al. 2014, The Messenger, 155, 29

Kovács, G., \& Kupi, G. 2007, A\&A, 462, 1007

Kunder, A., \& Chaboyer, B. 2009, AJ, 137, 4478

Kunder, A., Rich, R. M., Storm, J., et al. 2016, ApJ, 821, L25 
Longmore, A. J., Dixon, R., Skillen, I., Jameson, R. F., \& Fernley, J. A. 1990, MNRAS, 247, 684

Lucas, P. W., Hoare, M. G., Longmore, A., et al. 2008, MNRAS, 391, 136

McWilliam, A., \& Zoccali, M. 2010, ApJ, 724, 1491

Minniti, D., Lucas, P. W., Emerson, J. P., et al. 2010, New Astron., 15, 433

Minniti, D., Saito, R. K., Alonso-García, J., Lucas, P. W., \& Hempel, M. 2011, ApJ, 733, L43

Navarrete, C., Contreras Ramos, R., Catelan, M., et al. 2015, A\&A, 577, A99

Navarrete, C., Catelan, M., Contreras Ramos, R., Gran, F. \& Alonso-Garia, J. 2016, Communication from the Konkoly Observatory, 105, 45

Nataf, D. M., Udalski, A., Gould, A., Fouqué, P., \& Stanek, K. Z. 2010, ApJ, 721, L28

Ness, M., Freeman, K., Athanassoula, E., et al. 2012, ApJ, 756, 22

Pietrukowicz, P., Udalski, A., Soszyński, I., et al. 2012, ApJ, 750, 169

Rattenbury, N. J., Mao, S., Sumi, T., \& Smith, M. C. 2007, MNRAS, 378, 1064

Richards, J. W., Starr, D. L., Butler, N. R., et al. 2011, ApJ, 733, 10
Saito, R. K., Zoccali, M., McWilliam, A., et al. 2011, AJ, 142, 76 Saito, R. K., Hempel, M., Minniti, D., et al. 2012a, A\&A, 537, A107 Saito, R. K., Minniti, D., Dias, B., et al. 2012b, A\&A, 544, A147 Samus, N. N., \& Durlevich, O. V. 2009, VizieR Online Data Catalog: B/gcvs Scargle, J. D., Norris, J. P., Jackson, B., \& Chiang, J. 2013, ApJ, 764, 167

Schultheis, M., Chen, B. Q., Jiang, B. W., et al. 2014, A\&A, 566, A120 Schwarzenberg-Czerny, A. 1989, MNRAS, 241, 153

Skrutskie, M. F., Cutri, R. M., Stiening, R., et al. 2006, AJ, 131, 1163

Soszyński, I., Udalski, A., Szymański, M. K., et al. 2014, Acta Astron., 64, 177

Udalski, A., Szymański, M. K., \& Szymański, G. 2015, Acta Astron., 65, 1

Valenti, E., Ferraro, F. R., \& Origlia, L. 2004, MNRAS, 351, 1204

Valenti, E., Origlia, L., \& Ferraro, F. R. 2005, MNRAS, 361, 272

Vanderplas, J., Connolly, A., Ivezić, Ž., \& Gray, A. 2012, in Conf. on Intelligent Data Understanding (CIDU), 47

Wegg, C., \& Gerhard, O. 2013, MNRAS, 435, 1874 


\section{Appendix A: List of VVV RRab variables}

Table A.1 lists the main parameters of the 1019 ab-type RR Lyrae stars discovered in this work. For each object we provide the VVV name, equatorial and Galactic coordinates, mean $K_{\mathrm{s}}$-band weighted-magnitude, period, amplitude, and heliocentric distance. In Table A.2 we list the VVV RR Lyrae matching variables in the General Catalogue of Variable Stars (GCVS).

Table A.1. VVV RRab variables.

\begin{tabular}{|c|c|c|c|c|c|c|c|c|}
\hline VVV ID & $\begin{array}{l}\text { RA (J2000.0) } \\
\text { hh:mm:ss.ss }\end{array}$ & $\begin{array}{c}\text { Dec (J2000.0) } \\
\text { dd:mm:ss.s }\end{array}$ & $\begin{array}{c}\ell \\
(\mathrm{deg})\end{array}$ & $\begin{array}{c}b \\
(\mathrm{deg})\end{array}$ & $\begin{array}{c}\left\langle K_{\mathrm{s}}\right\rangle \\
(\mathrm{mag})\end{array}$ & $\begin{array}{c}P \\
\text { (days) }\end{array}$ & $\begin{array}{l}\text { Amplitude } \\
\text { (mag) }\end{array}$ & $\begin{array}{c}d \\
(\mathrm{kpc})\end{array}$ \\
\hline J175459.11-413137.1 & $17: 54: 59.11$ & $-41: 31: 37.1$ & -9.908 & -8.039 & 14.641 & 0.472833 & 0.30 & 10.0 \\
\hline J175601.56-412549.0 & $17: 56: 01.56$ & $-41: 25: 49.0$ & -9.727 & -8.161 & 13.998 & 0.656728 & 0.26 & 8.8 \\
\hline J175609.49-410752.7 & $17: 56: 09.49$ & $-41: 07: 52.7$ & -9.451 & -8.037 & 14.009 & 0.541568 & 0.37 & 8.0 \\
\hline $\mathrm{J} 175628.40-411745.8$ & $17: 56: 28.40$ & $-41: 17: 45.8$ & -9.567 & -8.169 & 13.651 & 0.517874 & 0.32 & 6.5 \\
\hline J175718.55-412623.3 & $17: 57: 18.55$ & $-41: 26: 23.3$ & -9.617 & -8.376 & 14.262 & 0.481343 & 0.35 & 8.4 \\
\hline J175727.54-414338.0 & $17: 57: 27.54$ & $-41: 43: 38.0$ & -9.858 & -8.540 & 14.875 & 0.589605 & 0.34 & 12.7 \\
\hline J175733.77-412426.2 & $17: 57: 33.77$ & $-41: 24: 26.2$ & -9.565 & -8.402 & 14.465 & 0.683723 & 0.23 & 11.2 \\
\hline $\mathrm{J} 175752.28-403611.6$ & $17: 57: 52.28$ & $-40: 36: 11.6$ & -8.826 & -8.063 & 14.261 & 0.569084 & 0.32 & 9.2 \\
\hline J175754.28-404601.9 & $17: 57: 54.28$ & $-40: 46: 01.9$ & -8.968 & -8.148 & 14.476 & 0.617486 & 0.34 & 10.7 \\
\hline J175819.66-414624.9 & $17: 58: 19.66$ & $-41: 46: 24.9$ & -9.820 & -8.704 & 15.012 & 0.599208 & 0.38 & 13.6 \\
\hline J175830.07-414442.0 & $17: 58: 30.07$ & $-41: 44: 42.0$ & -9.778 & -8.719 & 14.348 & 0.671317 & 0.27 & 10.5 \\
\hline J175837.43-411752.1 & $17: 58: 37.43$ & $-41: 17: 52.1$ & -9.371 & -8.523 & 14.134 & 0.662357 & 0.28 & 9.4 \\
\hline J175851.82-414716.9 & $17: 58: 51.82$ & $-41: 47: 16.9$ & -9.784 & -8.799 & 14.473 & 0.628840 & 0.26 & 10.8 \\
\hline J175852.19-412131.5 & $17: 58: 52.19$ & $-41: 21: 31.5$ & -9.402 & -8.593 & 13.769 & 0.637263 & 0.26 & 7.7 \\
\hline J175854.86-412915.7 & $17: 58: 54.86$ & $-41: 29: 15.7$ & -9.513 & -8.663 & 14.283 & 0.474424 & 0.30 & 8.5 \\
\hline J175923.54-400457.8 & $17: 59: 23.54$ & $-40: 04: 57.8$ & -8.224 & -8.065 & 13.831 & 0.484907 & 0.32 & 6.9 \\
\hline J175948.43-403817.5 & $17: 59: 48.43$ & $-40: 38: 17.5$ & -8.678 & -8.402 & 15.035 & 0.392449 & 0.36 & 11.0 \\
\hline J175955.47-403207.4 & $17: 59: 55.47$ & $-40: 32: 07.4$ & -8.576 & -8.372 & 14.506 & 0.571716 & 0.30 & 10.4 \\
\hline $\mathrm{J} 180024.80-415822.5$ & $18: 00: 24.80$ & $-41: 58: 22.5$ & -9.808 & -9.140 & 14.087 & 0.578781 & 0.29 & 8.6 \\
\hline $\mathrm{J} 180025.54-405436.0$ & $18: 00: 25.54$ & $-40: 54: 36.0$ & -8.862 & -8.635 & 14.633 & 0.508143 & 0.28 & 10.4 \\
\hline $\mathrm{J} 180025.80-403739.7$ & $18: 00: 25.80$ & $-40: 37: 39.7$ & -8.611 & -8.500 & 14.594 & 0.548249 & 0.34 & 10.6 \\
\hline $\mathrm{J} 180031.75-400913.3$ & 18:00:31.75 & $-40: 09: 13.3$ & -8.181 & -8.290 & 14.412 & 0.567270 & 0.25 & 9.9 \\
\hline J180038.48-410734.9 & 18:00:38.48 & $-41: 07: 34.9$ & -9.035 & -8.774 & 14.036 & 0.489627 & 0.28 & 7.6 \\
\hline J180039.53-400902.4 & $18: 00: 39.53$ & $-40: 09: 02.4$ & -8.167 & -8.310 & 13.515 & 0.649705 & 0.31 & 6.9 \\
\hline $\mathrm{J} 180041.35-411613.9 *$ & 18:00:41.35 & $-41: 16: 13.9$ & -9.158 & -8.851 & 14.182 & 0.629759 & 0.36 & 9.4 \\
\hline J180043.58-393334.9 & 18:00:43.58 & $-39: 33: 34.9$ & -7.637 & -8.037 & 14.305 & 0.600413 & 0.34 & 9.7 \\
\hline $\mathrm{J} 180047.28-420358.3 *$ & $18: 00: 47.28$ & $-42: 03: 58.3$ & -9.858 & -9.246 & 13.219 & 0.604051 & 0.28 & 5.8 \\
\hline J180051.02-395145.4 & $18: 00: 51.02$ & $-39: 51: 45.4$ & -7.894 & -8.204 & 13.298 & 0.806959 & 0.24 & 7.0 \\
\hline $\mathrm{J} 180052.96-411645.6$ & 18:00:52.96 & $-41: 16: 45.6$ & -9.149 & -8.887 & 14.448 & 0.598466 & 0.31 & 10.4 \\
\hline $\mathrm{J} 180053.51-394347.3$ & $18: 00: 53.51$ & $-39: 43: 47.3$ & -7.772 & -8.147 & 14.244 & 0.538057 & 0.34 & 8.9 \\
\hline J180058.03-412928.6 & $18: 00: 58.03$ & $-41: 29: 28.6$ & -9.330 & -9.002 & 15.033 & 0.495587 & 0.34 & 12.4 \\
\hline J180059.97-401819.4 & 18:00:59.97 & $-40: 18: 19.4$ & -8.273 & -8.441 & 14.301 & 0.498925 & 0.33 & 8.8 \\
\hline J180117.17-393923.0 & 18:01:17.17 & $-39: 39: 23.0$ & -7.670 & -8.178 & 13.988 & 0.534302 & 0.34 & 7.8 \\
\hline J180126.10-410043.4 & 18:01:26.10 & $-41: 00: 43.4$ & -8.861 & -8.851 & 14.418 & 0.585954 & 0.27 & 10.1 \\
\hline J180129.07-395817.7 & $18: 01: 29.07$ & $-39: 58: 17.7$ & -7.931 & -8.363 & 14.493 & 0.583019 & 0.23 & 10.5 \\
\hline J180133.14-420003.9 & 18:01:33.14 & $-42: 00: 03.9$ & -9.732 & -9.340 & 14.146 & 0.448021 & 0.32 & 7.7 \\
\hline J180136.04-415026.5 & $18: 01: 36.04$ & $-41: 50: 26.5$ & -9.584 & -9.272 & 14.728 & 0.560897 & 0.31 & 11.5 \\
\hline $\mathrm{J} 180138.52-395238.8 *$ & $18: 01: 38.52$ & $-39: 52: 38.8$ & -7.833 & -8.344 & 14.184 & 0.456159 & 0.22 & 7.9 \\
\hline J180138.91-412209.0 & $18: 01: 38.91$ & $-41: 22: 09.0$ & -9.159 & -9.056 & 13.779 & 0.572194 & 0.27 & 7.3 \\
\hline J180142.05-393258.3 & 18:01:42.05 & $-39: 32: 58.3$ & -7.537 & -8.197 & 15.202 & 0.566707 & 0.27 & 14.5 \\
\hline J180203.53-400432.9 & $18: 02: 03.53$ & $-40: 04: 32.9$ & -7.971 & -8.509 & 14.503 & 0.526078 & 0.23 & 9.9 \\
\hline J180206.50-390151.6 & $18: 02: 06.50$ & $-39: 01: 51.6$ & -7.039 & -8.018 & 14.054 & 0.713638 & 0.22 & 9.4 \\
\hline J180207.98-400449.7 & 18:02:07.98 & $-40: 04: 49.7$ & -7.968 & -8.524 & 14.109 & 0.569726 & 0.25 & 8.6 \\
\hline $\mathrm{J} 180214.87-412731.0$ & 18:02:14.87 & $-41: 27: 31.0$ & -9.185 & -9.197 & 14.455 & 0.535828 & 0.27 & 9.8 \\
\hline J180214.90-391059.2 & $18: 02: 14.90$ & $-39: 10: 59.2$ & -7.161 & -8.115 & 13.910 & 0.500065 & 0.27 & 7.3 \\
\hline J180218.28-413322.1* & $18: 02: 18.28$ & $-41: 33: 22.1$ & -9.267 & -9.253 & 13.345 & 0.678049 & 0.24 & 6.5 \\
\hline J180222.40-412829.4 & $18: 02: 22.40$ & $-41: 28: 29.4$ & -9.188 & -9.226 & 13.970 & 0.756624 & 0.31 & 9.3 \\
\hline J180242.19-393612.4* & $18: 02: 42.19$ & $-39: 36: 12.4$ & -7.491 & -8.393 & 14.712 & 0.628354 & 0.31 & 12.1 \\
\hline J180252.00-401219.3 & $18: 02: 52.00$ & $-40: 12: 19.3$ & -8.012 & -8.707 & 13.773 & 0.621813 & 0.35 & 7.6 \\
\hline J180259.55-404442.6 & $18: 02: 59.55$ & $-40: 44: 42.6$ & -8.482 & -8.983 & 14.299 & 0.472170 & 0.42 & 8.5 \\
\hline J180307.75-421805.3 & 18:03:07.75 & $-42: 18: 05.3$ & -9.860 & -9.739 & 15.222 & 0.660982 & 0.37 & 15.9 \\
\hline J180314.24-411102.7 & $18: 03: 14.24$ & $-41: 11: 02.7$ & -8.851 & -9.231 & 15.214 & 0.507123 & 0.32 & 13.7 \\
\hline J180318.09-384242.0 & 18:03:18.09 & $-38: 42: 42.0$ & -6.643 & -8.070 & 14.053 & 0.487555 & 0.29 & 7.7 \\
\hline J180318.38-383758.1 & $18: 03: 18.38$ & $-38: 37: 58.1$ & -6.573 & -8.033 & 13.474 & 0.711698 & 0.27 & 7.1 \\
\hline J180318.97-412248.6 & 18:03:18.97 & $-41: 22: 48.6$ & -9.019 & -9.337 & 13.357 & 0.746910 & 0.24 & 6.9 \\
\hline J180319.85-384537.6 & 18:03:19.85 & $-38: 45: 37.6$ & -6.684 & -8.098 & 13.943 & 0.671998 & 0.29 & 8.7 \\
\hline J180324.07-405300.9 & $18: 03: 24.07$ & $-40: 53: 00.9$ & -8.568 & -9.117 & 13.568 & 0.657026 & 0.28 & 7.1 \\
\hline J180329.14-404403.2 & $18: 03: 29.14$ & $-40: 44: 03.2$ & -8.427 & -9.061 & 13.299 & 0.745234 & 0.32 & 6.7 \\
\hline
\end{tabular}

Notes. VVV IDs marked with a single asterisk are the objects matching a variable in the General Cataloge of Variable Stars (GCVS). The corresponding GCVS names are presented in Table A.2. Three RRab stars previously discovered by OGLE-IV are marked with double asterisks, namely: J181632.17-334319.8 = OGLE-BLG-RRLYR-35577, J181727.29-335532.1 = OGLE-BLG-RRLYR-35765 and J181745.11-333025.2 = OGLE-BLG-RRLYR-35810. 
Table A.1. continued.

\begin{tabular}{|c|c|c|c|c|c|c|c|c|}
\hline VVV ID & $\begin{array}{c}\text { RA (J2000.0) } \\
\text { hh:mm:ss.ss }\end{array}$ & $\begin{array}{c}\text { Dec (J2000.0) } \\
\text { dd:mm:ss.s }\end{array}$ & $\begin{array}{c}\ell \\
(\mathrm{deg}) \\
\end{array}$ & $\begin{array}{c}b \\
(\mathrm{deg}) \\
\end{array}$ & $\begin{array}{l}\left\langle K_{\mathrm{s}}\right\rangle \\
(\mathrm{mag}) \\
\end{array}$ & $\begin{array}{c}P \\
\text { (days) } \\
\end{array}$ & $\begin{array}{l}\text { Amplitude } \\
\text { (mag) }\end{array}$ & $\begin{array}{c}d \\
(\mathrm{kpc})\end{array}$ \\
\hline J180334.27-421303.7 & $18: 03: 34.27$ & $-42: 13: 03.7$ & -9.746 & -9.772 & 15.045 & 0.523470 & 0.34 & 12.9 \\
\hline J180335.31-395348.3 & 18:03:35.31 & $-39: 53: 48.3$ & -7.671 & -8.682 & 13.765 & 0.539700 & 0.29 & 7.1 \\
\hline $\mathrm{J} 180342.46-392615.5 *$ & 18:03:42.46 & $-39: 26: 15.5$ & -7.251 & -8.484 & 14.093 & 0.390447 & 0.41 & 6.9 \\
\hline J180345.50-420853.7 & $18: 03: 45.50$ & $-42: 08: 53.7$ & -9.668 & -9.770 & 14.076 & 0.511941 & 0.33 & 8.0 \\
\hline J180346.93-390232.0 & 18:03:46.93 & $-39: 02: 32.0$ & -6.892 & -8.310 & 14.304 & 0.508466 & 0.31 & 8.9 \\
\hline $\mathrm{J} 180351.95-390948.3 *$ & 18:03:51.95 & $-39: 09: 48.3$ & -6.992 & -8.381 & 13.844 & 0.534605 & 0.30 & 7.3 \\
\hline J180356.13-400120.6 & 18:03:56.13 & $-40: 01: 20.6$ & -7.751 & -8.800 & 14.646 & 0.543759 & 0.31 & 10.8 \\
\hline J180400.42-395743.4 & 18:04:00.42 & $-39: 57: 43.4$ & -7.691 & -8.783 & 14.005 & 0.562756 & 0.30 & 8.1 \\
\hline J180407.52-405051.7 & 18:04:07.52 & $-40: 50: 51.7$ & -8.471 & -9.221 & 14.442 & 0.534985 & 0.32 & 9.7 \\
\hline J180407.93-410727.3 & 18:04:07.93 & $-41: 07: 27.3$ & -8.717 & -9.352 & 14.227 & 0.513896 & 0.31 & 8.6 \\
\hline J180413.93-383310.2 & 18:04:13.93 & $-38: 33: 10.2$ & -6.415 & -8.154 & 14.822 & 0.496739 & 0.37 & 11.2 \\
\hline J180415.38-402140.9 & 18:04:15.38 & $-40: 21: 40.9$ & -8.024 & -9.014 & 14.265 & 0.534248 & 0.37 & 8.9 \\
\hline J180416.19-390041.6* & 18:04:16.19 & $-39: 00: 41.6$ & -6.819 & -8.378 & 13.632 & 0.535857 & 0.24 & 6.6 \\
\hline J180424.81-382539.8 & 18:04:24.81 & $-38: 25: 39.8$ & -6.287 & -8.126 & 13.892 & 0.437742 & 0.29 & 6.7 \\
\hline $\mathrm{J} 180426.60-420755.7$ & $18: 04: 26.60$ & $-42: 07: 55.7$ & -9.593 & -9.875 & 14.598 & 0.560774 & 0.26 & 10.8 \\
\hline J180427.83-383712.3 & $18: 04: 27.83$ & $-38: 37: 12.3$ & -6.453 & -8.226 & 15.328 & 0.460588 & 0.26 & 13.8 \\
\hline J180429.41-404354.8 & 18:04:29.41 & $-40: 43: 54.8$ & -8.334 & -9.227 & 14.112 & 0.485555 & 0.35 & 7.9 \\
\hline J180431.95-411700.5* & 18:04:31.95 & $-41: 17: 00.5$ & -8.824 & -9.493 & 13.393 & 0.541703 & 0.30 & 5.9 \\
\hline J180432.08-393840.2 & 18:04:32.08 & $-39: 38: 40.2$ & -7.359 & -8.723 & 15.659 & 0.439425 & 0.39 & 15.8 \\
\hline J180433.29-382919.5 & 18:04:33.29 & $-38: 29: 19.5$ & -6.328 & -8.179 & 14.376 & 0.465643 & 0.34 & 8.8 \\
\hline J180434.24-393614.6 & $18: 04: 34.24$ & $-39: 36: 14.6$ & -7.320 & -8.710 & 14.395 & 0.474892 & 0.31 & 8.9 \\
\hline J180439.18-394556.3 & 18:04:39.18 & $-39: 45: 56.3$ & -7.456 & -8.800 & 14.150 & 0.551682 & & 8.6 \\
\hline $\mathrm{J} 180441.26-421253.4$ & 18:04:41.26 & $-42: 12: 53.4$ & -9.646 & -9.9 & 14.031 & 0.638696 & & 8.8 \\
\hline $\mathrm{J} 180457.98-383144.8$ & 18:04:57.98 & $-38: 31: 44.8$ & -6.325 & -8.269 & 14.543 & 0.592550 & 0.33 & 10.8 \\
\hline $\mathrm{J} 180500.80-415330.1$ & 18:05:00.80 & $-41: 53: 30.1$ & -9.327 & -9.857 & 14.000 & 0.625493 & 0.29 & 8.6 \\
\hline J180501.55-413619.8 & 18:05:01.55 & $-41: 36: 19.8$ & -9.069 & -9.725 & 13.785 & 0.548456 & 0.29 & 7.2 \\
\hline J180506.94-411744.6 & 18:05:06.94 & $-41: 17: 44.6$ & -8.783 & -9.596 & 13.842 & 0.786720 & 0.28 & 9.0 \\
\hline $\mathrm{J} 180508.45-385408.1$ & 18:05:08.45 & $-38: 54: 08.1$ & -6.641 & -8.476 & 15.175 & 0.485512 & 0.24 & 13.2 \\
\hline J180514.45-405946.8 & $18: 05: 14.45$ & $-40: 59: 46.8$ & -8.504 & -9.477 & 14.254 & 0.543839 & 0.34 & 9.0 \\
\hline J180517.94-394136.4 & 18:05:17.94 & $-39: 41: 36.4$ & -7.333 & -8.876 & 13.974 & 0.562658 & 0.26 & 8.0 \\
\hline $\mathrm{J} 180521.73-381552.2$ & $18: 05: 21.73$ & $-38: 15: 52.2$ & -6.052 & -8.213 & 14.580 & 0.577634 & 0.32 & 10.9 \\
\hline J180522.12-392215.3 & $18: 05: 22.12$ & $-39: 22: 15.3$ & -7.038 & -8.736 & 14.129 & 0.528594 & 0.26 & 8.3 \\
\hline J180522.92-384605.4 & $18: 05: 22.92$ & $-38: 46: 05.4$ & -6.499 & -8.454 & 14.095 & 0.517518 & 0.37 & 8.1 \\
\hline J180523.00-415030.5 & $18: 05: 23.00$ & $-41: 50: 30.5$ & -9.250 & -9.894 & 15.224 & 0.582245 & 0.33 & 14.9 \\
\hline $\mathrm{J} 180534.80-421647.8 *$ & $18: 05: 34.80$ & $-42: 16: 47.8$ & -9.627 & -10.130 & 14.132 & 0.485058 & 0.23 & 8.0 \\
\hline J180536.41-400914.8 & 18:05:36.41 & $-40: 09: 14.8$ & -7.716 & -9.144 & 14.196 & 0.540141 & 0.24 & 8.7 \\
\hline J180537.74-421241.6 & $18: 05: 37.74$ & $-42: 12: 41.6$ & -9.561 & -10.107 & 14.584 & 0.510837 & 0.29 & 10.2 \\
\hline J180547.12-395054.3 & $18: 05: 47.12$ & $-39: 50: 54.3$ & -7.427 & -9.031 & 14.787 & 0.467360 & 0.35 & 10.7 \\
\hline J180547.69-401955.6 & 18:05:47.69 & $-40: 19: 55.6$ & -7.859 & -9.259 & 14.865 & 0.615358 & 0.19 & 12.9 \\
\hline $\mathrm{J} 180548.77-421314.0 *$ & $18: 05$ & $-42: 13: 14.0$ & -9.553 & -10.141 & 14.232 & 0.460162 & 0.35 & 8.1 \\
\hline J180553.06-382624.7 & 18:05:53.06 & $-38: 26: 24.7$ & -6.160 & -8.386 & 14.364 & 0.557777 & 0.34 & 9.6 \\
\hline $\mathrm{J} 180555.71-421832.3$ & $18: 05: 55.71$ & $-42: 18: 32.3$ & -9.622 & -10.201 & 14.460 & 0.491162 & 0.37 & 9.4 \\
\hline J180556.04-394418.1* & 18:05:56.04 & $-39: 44: 18.1$ & -7.315 & -9.004 & 14.151 & 0.563990 & 0.33 & 8.7 \\
\hline J180556.80-420223.7 & $18: 05: 56.80$ & $-42: 02: 23.7$ & -9.378 & -10.079 & 14.230 & 0.642893 & 0.25 & 9.7 \\
\hline J180603.18-393014.7 & 18:06:03.18 & $-39: 30: 14.7$ & -7.094 & -8.915 & 14.488 & 0.545768 & 0.28 & 10.1 \\
\hline J180608.88-380449.7 & 18:06:08.88 & $-38: 04: 49.7$ & -5.815 & -8.262 & 14.020 & 0.499059 & 0.24 & 7.6 \\
\hline J180610.23-381015.4 & 18:06:10.23 & $-38: 10: 15.4$ & -5.893 & -8.308 & 14.120 & 0.471076 & 0.20 & 7.8 \\
\hline J180610.70-392742.8 & 18:06:10.70 & $-39: 27: 42.8$ & -7.045 & -8.917 & 14.252 & 0.486107 & 0.31 & 8.4 \\
\hline J180611.40-375419.0 & 18:06:11.40 & $-37: 54: 19.0$ & -5.655 & -8.186 & 15.152 & 0.494748 & 0.17 & 13.2 \\
\hline $\mathrm{J} 180628.50-375807.5$ & $18: 06: 28.50$ & $-37: 58: 07.5$ & -5.685 & -8.266 & 14.204 & 0.505802 & 0.28 & 8.4 \\
\hline J180631.39-400108.9 & 18:06:31.39 & $-40: 01: 08.9$ & -7.512 & -9.236 & 15.042 & 0.468733 & 0.31 & 12.1 \\
\hline $\mathrm{J} 180636.51-393543.8 *$ & $18: 06: 36.51$ & $-39: 35: 43.8$ & -7.125 & -9.052 & 12.712 & 0.537527 & 0.27 & 4.2 \\
\hline J180640.53-403848.1 & $18: 06: 40.53$ & $-40: 38: 48.1$ & -8.062 & -9.554 & 15.580 & 0.529916 & 0.30 & 16.8 \\
\hline $\mathrm{J} 180642.69-410352.1 *$ & 18:06:42.69 & $-41: 03: 52.1$ & -8.434 & -9.754 & 14.264 & 0.602442 & 0.23 & 9.5 \\
\hline J180645.52-394908.3 & $18: 06: 45.52$ & $-39: 49: 08.3$ & -7.312 & -9.182 & 14.716 & 0.565042 & 0.24 & 11.5 \\
\hline J180646.23-394644.8 & $18: 06: 46.23$ & $-39: 46: 44.8$ & -7.275 & -9.166 & 13.800 & 0.912051 & 0.24 & 9.5 \\
\hline J180649.01-414058.6 & $18: 06: 49.01$ & $-41: 40: 58.6$ & -8.981 & -10.058 & 14.295 & 0.516761 & 0.30 & 8.9 \\
\hline $\mathrm{J} 180656.21-385517.7 *$ & 18:06:56.21 & $-38: 55: 17.7$ & -6.492 & -8.793 & 13.833 & 0.682757 & 0.24 & 8.3 \\
\hline J180658.83-412738.2 & $18: 06: 58.83$ & $-41: 27: 38.2$ & -8.766 & -9.982 & 14.638 & 0.492065 & 0.30 & 10.2 \\
\hline J180701.43-383135.1 & 18:07:01.43 & $-38: 31: 35.1$ & -6.131 & -8.623 & 14.557 & 0.522870 & 0.36 & 10.2 \\
\hline $\mathrm{J} 180712.91-380835.2$ & 18:07:12.91 & $-38: 08: 35.2$ & -5.771 & -8.477 & 14.577 & 0.424558 & 0.26 & 9.2 \\
\hline
\end{tabular}


Table A.1. continued.

\begin{tabular}{|c|c|c|c|c|c|c|c|c|}
\hline VVV ID & $\begin{array}{l}\text { RA (J2000.0) } \\
\text { hh:mm:ss.ss }\end{array}$ & $\begin{array}{c}\text { Dec (J2000.0) } \\
\text { dd:mm:ss.s }\end{array}$ & $\begin{array}{c}\ell \\
(\operatorname{deg})\end{array}$ & $\begin{array}{c}b \\
(\mathrm{deg})\end{array}$ & $\begin{array}{l}\left\langle K_{\mathrm{s}}\right\rangle \\
(\mathrm{mag})\end{array}$ & $\begin{array}{c}P \\
\text { (days) }\end{array}$ & $\begin{array}{l}\text { Amplitude } \\
\text { (mag) }\end{array}$ & $\begin{array}{c}d \\
(\mathrm{kpc})\end{array}$ \\
\hline J180713.57-413121.7 & 18:07:13.57 & $-41: 31: 21.7$ & -8.801 & -10.052 & 14.443 & 0.519276 & 0.21 & 9.6 \\
\hline J180715.53-382158.1 & 18:07:15.53 & $-38: 21: 58.1$ & -5.966 & -8.589 & 14.914 & 0.445800 & 0.23 & 11.1 \\
\hline J180718.20-400044.4 & 18:07:18.20 & $-40: 00: 44.4$ & -7.436 & -9.365 & 14.176 & 0.729320 & 0.18 & 10.1 \\
\hline J180720.63-410313.3 & 18:07:20.63 & $-41: 03: 13.3$ & -8.368 & -9.854 & 13.387 & 0.816446 & 0.17 & 7.3 \\
\hline J180721.68-401440.9 & $18: 07: 21.68$ & $-40: 14: 40.9$ & -7.639 & -9.483 & 14.370 & 0.491681 & 0.28 & 9.0 \\
\hline $\mathrm{J} 180728.98-374352.8$ & 18:07:28.98 & $-37: 43: 52.8$ & -5.378 & -8.330 & 14.306 & 0.611396 & 0.22 & 9.8 \\
\hline J180731.38-392338.6 & 18:07:31.38 & $-39: 23: 38.6$ & -6.862 & -9.114 & 14.278 & 0.521222 & 0.27 & 8.9 \\
\hline J180736.67-413337.7 & 18:07:36.67 & $-41: 33: 37.7$ & -8.801 & -10.133 & 13.537 & 0.622886 & 0.19 & 6.8 \\
\hline J180740.20-403538.9 & 18:07:40.20 & $-40: 35: 38.9$ & -7.926 & -9.697 & 14.811 & 0.704642 & 0.30 & 13.5 \\
\hline J180743.06-411212.5 & 18:07:43.06 & $-41: 12: 12.5$ & -8.470 & -9.986 & 13.700 & 0.605539 & 0.23 & 7.3 \\
\hline J180755.27-400553.7 & 18:07:55.27 & $-40: 05: 53.7$ & -7.457 & -9.509 & 15.367 & 0.583989 & 0.29 & 16.0 \\
\hline J180756.11-383429.3 & 18:07:56.11 & $-38: 34: 29.3$ & -6.090 & -8.803 & 14.206 & 0.530721 & 0.26 & 8.7 \\
\hline J180758.88-370052.1 & 18:07:58.88 & $-37: 00: 52.1$ & -4.692 & -8.081 & 14.822 & 0.487522 & 0.25 & 11.1 \\
\hline $\mathrm{J} 180800.40-374514.8 *$ & 18:08:00.40 & $-37: 45: 14.8$ & -5.350 & -8.432 & 13.830 & 0.488926 & 0.34 & 6.9 \\
\hline J180800.66-392540.5 & 18:08:00.66 & $-39: 25: 40.5$ & -6.847 & -9.214 & 14.165 & 0.499715 & 0.32 & 8.2 \\
\hline J180801.62-371510.7 & 18:08:01.62 & $-37: 15: 10.7$ & -4.900 & -8.201 & 14.196 & 0.472306 & 0.35 & 8.1 \\
\hline $\mathrm{J} 180804.68-372205.5 *$ & 18:08:04.68 & $-37: 22: 05.5$ & -4.998 & -8.264 & 13.697 & 0.530400 & 0.31 & 6.8 \\
\hline J180818.85-403316.4 & 18:08:18.85 & $-40: 33: 16.4$ & -7.833 & -9.787 & 13.742 & 0.746960 & 0.27 & 8.3 \\
\hline J180820.91-391057.5 & 18:08:20.91 & $-39: 10: 57.5$ & -6.597 & -9.157 & 13.229 & 0.756601 & 0.28 & 6.5 \\
\hline J180822.15-385446.0 & 18:08:22.15 & $-38: 54: 46.0$ & -6.353 & -9.036 & 14.812 & 0.567469 & 0.33 & 12.0 \\
\hline J180823.21-372833.8 & $18: 08: 23.21$ & $-37: 28: 33.8$ & -5.066 & -8.368 & 14.381 & 0.464149 & 0.30 & 8.8 \\
\hline J180827.85-382240.2 & 18:08:27.85 & $-38: 22: 40.2$ & -5.865 & -8.803 & 14.199 & 0.501215 & 0.34 & 8.4 \\
\hline J180832.53-405718.1 & $18: 08: 32.53$ & $-40: 57: 18.1$ & -8.173 & -10.01 & 13.929 & 0.616637 & 0.33 & 8.2 \\
\hline J180836.95-395145.5 & 18:08:36.95 & $-39: 51: 45.5$ & -7.183 & -9.518 & 14.540 & 0.478270 & 0.32 & 9.6 \\
\hline J180836.98-405522.9 & 18:08:36.98 & $-40: 55: 22.9$ & -8.138 & -10.007 & 14.410 & 0.736323 & 0.36 & 11.4 \\
\hline J180838.52-411116.8 & $18: 08: 38.52$ & $-41: 11: 16.8$ & -8.375 & -10.133 & 13.506 & 0.562019 & 0.27 & 6.4 \\
\hline J180840.45-371438.0 & 18:08:40.45 & $-37: 14: 38.0$ & -4.831 & -8.310 & 15.509 & 0.497124 & 0.35 & 15.7 \\
\hline $\mathrm{J} 180842.70-385557.8 *$ & $18: 08: 42.70$ & $-38: 55: 57.8$ & -6.339 & -9.104 & 13.293 & 0.447351 & 0.34 & 5.1 \\
\hline J180844.36-411703.5 & 18:08:44.36 & $-41: 17: 03.5$ & -8.453 & -10.194 & 14.678 & 0.493513 & 0.26 & 10.5 \\
\hline J180846.34-395529.5 & 18:08:46.34 & $-39: 55: 29.5$ & -7.225 & -9.574 & 15.188 & 0.464755 & 0.31 & 13.0 \\
\hline J180849.63-392831.6 & $18: 08: 49.63$ & $-39: 28: 31.6$ & -6.816 & -9.375 & 14.274 & 0.749614 & 0.32 & 10.8 \\
\hline $\mathrm{J} 180851.52-363732.8$ & 18:08:51.52 & $-36: 37: 32.8$ & -4.262 & -8.053 & 15.464 & 0.495692 & 0.30 & 15.3 \\
\hline $\mathrm{J} 180855.13-401650.0$ & 18:08:55.13 & $-40: 16: 50.0$ & -7.532 & -9.763 & 14.424 & 0.577731 & 0.23 & 10.1 \\
\hline J180856.08-372615.5 & 18:08:56.08 & $-37: 26: 15.5$ & -4.980 & -8.447 & 14.129 & 0.481726 & 0.25 & 7.9 \\
\hline J180858.57-393240.9 & $18: 08: 58.57$ & $-39: 32: 40.9$ & -6.865 & -9.433 & 16.293 & 0.526432 & 0.36 & 23.6 \\
\hline J180859.74-371655.7 & 18:08:59.74 & $-37: 16: 55.7$ & -4.835 & -8.385 & 13.781 & 0.505906 & 0.23 & 6.9 \\
\hline J180906.12-385330.9 & 18:09:06.12 & $-38: 53: 30.9$ & -6.267 & -9.152 & 14.263 & 0.443135 & 0.34 & 8.1 \\
\hline J180907.77-403218.2 & 18:09:07.77 & $-40: 32: 18.2$ & -7.746 & -9.917 & 15.100 & 0.363689 & 0.36 & 10.9 \\
\hline $\mathrm{J} 180908.23-405758.1 *$ & 18:09:08.23 & $-40: 57: 58.1$ & -8.131 & -10.114 & 14.238 & 0.485446 & 0.28 & 8.4 \\
\hline J180911.36-373841.8 & 18:09:11.36 & $-37: 38: 41.8$ & -5.141 & -8.588 & 14.843 & 0.640246 & 0.33 & 13.0 \\
\hline J180920.21-383353.6 & 18:09:20.21 & $-38: 33: 53.6$ & -5.952 & -9.041 & 13.288 & 0.606127 & 0.26 & 6.0 \\
\hline J180921.59-392419.5 & 18:09:21.59 & $-39: 24: 19.5$ & -6.705 & -9.434 & 14.482 & 0.693621 & 0.31 & 11.4 \\
\hline J180921.59-404349.4* & 18:09:21.59 & $-40: 43: 49.4$ & -7.899 & -10.044 & 14.400 & 0.660589 & 0.30 & 10.7 \\
\hline J180922.87-390927.8 & 18:09:22.87 & $-39: 09: 27.8$ & -6.480 & -9.323 & 13.159 & 0.809363 & 0.25 & 6.5 \\
\hline J180924.54-401447.4 & 18:09:24.54 & $-40: 14: 47.4$ & -7.458 & -9.830 & 14.258 & 0.568469 & 0.28 & 9.2 \\
\hline J180926.28-365108.5 & 18:09:26.28 & $-36: 51: 08.5$ & -4.409 & -8.262 & 14.307 & 0.567260 & 0.28 & 9.4 \\
\hline $\mathrm{J} 180926.56-390932.1 *$ & 18:09:26.56 & $-39: 09: 32.1$ & -6.476 & -9.334 & 14.269 & 0.439199 & 0.35 & 8.1 \\
\hline J180927.18-364036.6 & 18:09:27.18 & $-36: 40: 36.6$ & -4.251 & -8.183 & 14.374 & 0.472331 & 0.34 & 8.8 \\
\hline J180927.63-383636.9 & 18:09:27.63 & $-38: 36: 36.9$ & -5.981 & -9.084 & 13.931 & 0.663033 & 0.28 & 8.5 \\
\hline J180929.82-363652.5 & 18:09:29.82 & $-36: 36: 52.5$ & -4.191 & -8.161 & 14.796 & 0.475975 & 0.23 & 10.9 \\
\hline J180942.17-365524.7 & 18:09:42.17 & $-36: 55: 24.7$ & -4.448 & -8.342 & 14.791 & 0.527415 & 0.22 & 11.4 \\
\hline J180945.01-401822.3 & 18:09:45.01 & $-40: 18: 22.3$ & -7.481 & -9.915 & 13.280 & 0.600875 & 0.22 & 5.9 \\
\hline $\mathrm{J} 180949.72-364838.9 *$ & 18:09:49.72 & $-36: 48: 38.9$ & -4.335 & -8.312 & 13.589 & 0.535395 & 0.27 & 6.4 \\
\hline J180951.68-392552.4 & 18:09:51.68 & $-39: 25: 52.4$ & -6.683 & -9.532 & 14.648 & 0.518374 & 0.18 & 10.6 \\
\hline J180952.44-361626.0 & 18:09:52.44 & $-36: 16: 26.0$ & -3.851 & -8.069 & 14.147 & 0.588984 & 0.32 & 8.9 \\
\hline J180958.42-380307.7 & 18:09:58.42 & $-38: 03: 07.7$ & -5.434 & -8.914 & 14.403 & 0.523034 & 0.25 & 9.4 \\
\hline J181002.67-383833.0 & 18:10:02.67 & $-38: 38: 33.0$ & -5.957 & -9.200 & 13.338 & 0.522999 & 0.25 & 5.6 \\
\hline J181011.78-384805.0 & 18:10:11.78 & $-38: 48: 05.0$ & -6.086 & -9.299 & 14.166 & 0.610139 & 0.20 & 9.2 \\
\hline J181013.25-405607.6 & $18: 10: 13.25$ & $-40: 56: 07.6$ & -8.008 & -10.282 & 14.903 & 0.503069 & 0.33 & 11.8 \\
\hline J181016.09-394207.7 & 18:10:16.09 & $-39: 42: 07.7$ & -6.890 & -9.726 & 14.677 & 0.514340 & 0.29 & 10.7 \\
\hline J181017.82-371220.7 & $18: 10: 17.82$ & $-37: 12: 20.7$ & -4.645 & -8.578 & 16.236 & 0.454275 & 0.45 & 21.2 \\
\hline
\end{tabular}


Table A.1. continued.

\begin{tabular}{|c|c|c|c|c|c|c|c|c|}
\hline VVV ID & $\begin{array}{c}\text { RA (J2000.0) } \\
\text { hh:mm:ss.ss }\end{array}$ & $\begin{array}{c}\text { Dec (J2000.0) } \\
\text { dd:mm:ss.s }\end{array}$ & $\begin{array}{c}\ell \\
(\mathrm{deg})\end{array}$ & $\begin{array}{c}b \\
(\mathrm{deg})\end{array}$ & $\begin{array}{l}\left\langle K_{\mathrm{s}}\right\rangle \\
(\mathrm{mag})\end{array}$ & $\begin{array}{c}P \\
\text { (days) }\end{array}$ & $\begin{array}{l}\text { Amplitude } \\
\text { (mag) }\end{array}$ & $\begin{array}{c}d \\
(\mathrm{kpc})\end{array}$ \\
\hline J181018.92-372946.2 & $18: 10: 18.92$ & $-37: 29: 46.2$ & -4.903 & -8.716 & 14.632 & 0.474201 & 0.25 & 10.0 \\
\hline $\mathrm{J} 181020.74-400650.8$ & $18: 10: 20.74$ & $-40: 06: 50.8$ & -7.255 & -9.928 & 14.434 & 0.531426 & 0.32 & 9.7 \\
\hline J181022.56-404726.1 & $18: 10: 22.56$ & $-40: 47: 26.1$ & -7.863 & -10.242 & 14.432 & 0.457733 & 0.30 & 8.9 \\
\hline $\mathrm{J} 181023.42-364335.2$ & $18: 10: 23.42$ & $-36: 43: 35.2$ & -4.207 & -8.372 & 14.146 & 0.652835 & 0.25 & 9.4 \\
\hline $\mathrm{J} 181025.95-375450.8$ & $18: 10: 25.95$ & $-37: 54: 50.8$ & -5.267 & -8.93 & 14.962 & 0.474816 & 0.36 & 11.7 \\
\hline J181027.91-362807.1 & 18:10:27.91 & $-36: 28: 07.1$ & -3.969 & -8.265 & 13.054 & 0.814930 & 0.29 & 6.2 \\
\hline J181031.13-383304.1 & $18: 10: 31.13$ & $-38: 33: 04.1$ & -5.832 & -9.24 & 14.284 & 0.580869 & 0.30 & 9.4 \\
\hline J181031.72-373419.9 & $18: 10: 31.72$ & $-37: 34: 19.9$ & -4.952 & -8.789 & 13.864 & 0.559572 & 0.23 & 7.5 \\
\hline J181034.26-393635.0 & $18: 10: 34.26$ & $-39: 36: 35.0$ & -6.780 & -9.735 & 14.194 & 0.576725 & 0.18 & 9.0 \\
\hline $\mathrm{J} 181035.41-370405.7$ & $18: 10: 35.41$ & $-37: 04: 05.7$ & -4.494 & -8.566 & 14.210 & 0.470757 & 0.35 & 8.1 \\
\hline J181036.69-393656.6 & $18: 10: 36.69$ & $-39: 36: 56.6$ & -6.782 & -9.745 & 14.743 & 0.598695 & 0.24 & 12.0 \\
\hline J181042.59-402037.5 & $18: 10: 42.59$ & $-40: 20: 37.5$ & -7.430 & -10.095 & 14.071 & 0.535613 & 0.36 & 8.1 \\
\hline J181042.93-384206.5 & $18: 10: 42.93$ & $-38: 42: 06.5$ & -5.949 & -9.343 & 13.997 & 0.608332 & 0.30 & 8.4 \\
\hline J181047.43-395154.1 & $18: 10: 47.43$ & $-39: 51: 54.1$ & -6.991 & -9.890 & 14.327 & 0.648496 & 0.33 & 10.2 \\
\hline J181053.31-385903.9 & $18: 10: 53.31$ & $-38: 59: 03.9$ & -6.188 & -9.503 & & 0.531237 & 0.34 & 12.9 \\
\hline J181058.42-403002.4 & $18: 10: 58.42$ & $-40: 30: 02.4$ & -7.549 & -10.211 & 14.172 & 0.728470 & 0.17 & 10.1 \\
\hline J181100.35-391234.4 & 18:11:00.35 & $-39: 12: 34.4$ & -6.380 & -9.627 & 14.900 & 0.471125 & 0.25 & 11.3 \\
\hline $\mathrm{J} 181100.42-402858.9$ & 18:11:00.42 & $-40: 28: 58.9$ & -7.530 & -10.209 & 14.518 & 0.543845 & 0.39 & 10.2 \\
\hline J181103.37-401047.8 & $18: 11: 03.37$ & $-40: 10: 47.8$ & -7.251 & -10.079 & 15.713 & 0.468878 & 0.36 & 16.8 \\
\hline $\mathrm{J} 181103.50-385054.5 *$ & $18: 11: 03.50$ & $-38: 50: 54.5$ & -6.050 & -9.470 & 13.990 & 0.557296 & 0.29 & 8.0 \\
\hline J181103.58-361356.5 & $18: 11: 03.58$ & $-36: 13: 56.5$ & -3.702 & -8.261 & 13.977 & 0.612775 & & 8.4 \\
\hline $\mathrm{J} 181106.66-403516.1$ & 18:11:06.66 & $-40: 35: 16.1$ & -7.616 & -10.274 & 14.6 & 0.596531 & & 11.6 \\
\hline J181106.98-370820.3 & 18:11:06.98 & $-37: 08: 20.3$ & -4.508 & -8.692 & 14.219 & 0.543658 & 0.32 & 8.8 \\
\hline J181108.98-381140.4 & 18:11:08.98 & $-38: 11: 40.4$ & -5.453 & -9.185 & 14.577 & 0.643468 & 0.30 & 11.5 \\
\hline J181109.39-373847.3 & 9.39 & $-37:$ & -4.960 & -8.5 & 14.213 & 0.6 & 0.27 & 9.7 \\
\hline $\mathrm{J} 181111.94-372125.4 *$ & 18:11:11.94 & $-37:$ & -4.696 & -8.807 & 13.6 & 0.53 & 0.30 & 6.7 \\
\hline J181113.24-400259.0 & $18: 11: 13.24$ & $-40:$ & -7.119 & -10.048 & 12.921 & 0.59 & 0.25 & 4.9 \\
\hline $\mathrm{J} 181115.73-362110.3 *$ & $18: 11: 15.73$ & $-36: 21: 10.3$ & -3.790 & -8.353 & 13.7 & 0.778931 & 0.28 & 8.4 \\
\hline J181118.17-400233.8 & $18: 11: 18.17$ & $-40: 02: 33.8$ & -7.106 & -10.058 & 13.756 & 0.438847 & 0.30 & 6.3 \\
\hline J181120.29-373100.3 & $18: 11: 20.29$ & $-37: 31: 00.3$ & -4.827 & -8.906 & 14.070 & 0.650893 & 0.21 & 9.0 \\
\hline J181128.98-371318.0 & $18: 11: 28.98$ & $-37: 13: 18.0$ & -4.548 & -8.795 & 14.232 & 0.545712 & 0.25 & 8.9 \\
\hline $\mathrm{J} 181130.26-354416.0$ & $18: 11: 30.26$ & $-35: 44: 16.0$ & -3.217 & -8.111 & 13.771 & 0.627778 & 0.31 & 7.7 \\
\hline J181131.38-382345.4 & $18: 11: 31.38$ & $-38: 23: 45.4$ & -5.600 & -9.343 & 14.302 & 0.675566 & 0.28 & 10.3 \\
\hline $\mathrm{J} 181133.39-392737.7 *$ & $18: 11: 33.39$ & $-39: 27: 37.7$ & -6.557 & -9.836 & 13.522 & 0.583897 & 0.25 & 6.5 \\
\hline J181134.17-394504.7 & $18: 11: 34.17$ & $-39: 45: 04.7$ & -6.819 & -9.971 & 14.188 & 0.588228 & 0.21 & 9.1 \\
\hline J181137.34-384605.6 & $18: 11: 37.34$ & $-38: 46: 0$ & -5.927 & -9.5 & 14.119 & 0.741625 & & 9.9 \\
\hline J181139.52-395655.1 & $18: 11: 39.52$ & $-39: 56: 55.1$ & -6.989 & -10.076 & 15.626 & 0.479881 & 0.48 & 16.3 \\
\hline J181140.66-385901.0 & 18:11:40.66 & $-38: 59: 01.0$ & -6.116 & -9.639 & 13.583 & 0.491210 & 0.22 & 6.1 \\
\hline J181144.18-372104.9 & $18: 11: 44.18$ & -37 : & -4.641 & -8.899 & 14.046 & 0.589150 & 0.13 & 8.5 \\
\hline $\mathrm{J} 181144.32-353244.2$ & $18: 11: 44.32$ & $-35: 32: 44.2$ & -3.023 & -8.064 & 13.740 & 0.657800 & 0.32 & 7.8 \\
\hline $\mathrm{J} 181145.07-392818.1$ & $18: 11: 45.07$ & $-39: 28: 18.1$ & -6.550 & -9.875 & 14.513 & 0.567688 & 0.27 & 10.4 \\
\hline J181145.56-391102.8 & $18: 11: 45.56$ & $-39: 11: 02.8$ & -6.289 & -9.745 & 14.579 & 0.520855 & 0.22 & 10.3 \\
\hline J181147.73-384114.9 & 18:11:47.73 & $-38: 41: 14.9$ & -5.838 & -9.524 & 14.679 & 0.672948 & 0.24 & 12.4 \\
\hline J181150.55-373047.4 & $18: 11: 50.55$ & $-37: 30: 47.4$ & -4.777 & -8.993 & 13.320 & 0.764924 & 0.21 & 6.9 \\
\hline J181151.47-370658.3 & 18:11:51.47 & $-37: 06: 58.3$ & -4.419 & -8.812 & 13.761 & 0.551910 & 0.26 & 7.1 \\
\hline J181157.07-375547.6 & $18: 11: 57.07$ & $-37: 55: 47.6$ & -5.142 & -9.203 & 14.714 & 0.543210 & 0.26 & 11.2 \\
\hline J181158.09-372919.2 & 18:11:58.09 & $-37: 29: 19.2$ & -4.743 & -9.004 & 14.085 & 0.845780 & 0.22 & 10.5 \\
\hline J181159.45-400019.7 & $18: 11: 59.45$ & $-40: 00: 19.7$ & -7.011 & -10.159 & 14.421 & 0.599790 & 0.23 & 10.3 \\
\hline $\mathrm{J} 181202.26-384356.4$ & $18: 12: 02.26$ & $-38: 43: 56.4$ & -5.857 & -9.586 & 14.512 & 0.558931 & 0.29 & 10.3 \\
\hline J181204.93-363129.9 & $18: 12: 04.93$ & $-36: 31: 29.9$ & -3.867 & -8.579 & 15.061 & 0.570047 & 0.24 & 13.6 \\
\hline J181206.01-391219.9 & $18: 12: 06.01$ & $-39: 12: 19.9$ & -6.278 & -9.813 & 14.420 & 0.563517 & 0.28 & 9.9 \\
\hline J181206.96-400454.7 & 18:12:06.96 & $-40: 04: 54.7$ & -7.069 & -10.215 & 14.324 & 0.552385 & 0.23 & 9.4 \\
\hline J181211.71-360342.4 & $18: 12: 11.71$ & $-36: 03: 42.4$ & -3.442 & -8.385 & 14.373 & 0.640176 & 0.24 & 10.4 \\
\hline $\mathrm{J} 181215.87-354437.1$ & $18: 12: 15.87$ & $-35: 44: 37.1$ & -3.150 & -8.250 & 14.420 & 0.539561 & 0.24 & 9.7 \\
\hline J181215.95-394830.3 & 18:12:15.95 & $-39: 48: 30.3$ & -6.808 & -10.116 & 14.806 & 0.492764 & 0.31 & 11.1 \\
\hline J181219.95-392207.4 & 18:12:19.95 & $-39: 22: 07.4$ & -6.405 & -9.928 & 14.883 & 0.576166 & 0.20 & 12.6 \\
\hline J181221.81-365216.9 & $18: 12: 21.81$ & $-36: 52: 16.9$ & -4.152 & -8.789 & 13.948 & 0.639795 & 0.20 & 8.4 \\
\hline $\mathrm{J} 181222.44-360134.7 *$ & $18: 12: 22.44$ & $-36: 01: 34.7$ & -3.393 & -8.401 & 14.393 & 0.537138 & 0.28 & 9.5 \\
\hline J181222.54-365726.0 & $18: 12: 22.54$ & $-36: 57: 26.0$ & -4.228 & -8.831 & 14.260 & 0.543886 & 0.31 & 9.0 \\
\hline $\mathrm{J} 181230.45-352109.1 *$ & $18: 12: 30.45$ & $-35: 21: 09.1$ & -2.777 & -8.113 & 13.850 & 0.485030 & 0.34 & 6.9 \\
\hline
\end{tabular}


Table A.1. continued.

\begin{tabular}{|c|c|c|c|c|c|c|c|c|}
\hline VVV ID & $\begin{array}{l}\text { RA (J2000.0) } \\
\text { hh:mm:ss.ss }\end{array}$ & $\begin{array}{c}\text { Dec (J2000.0) } \\
\text { dd:mm:ss.s }\end{array}$ & $\begin{array}{c}\ell \\
(\mathrm{deg})\end{array}$ & $\begin{array}{c}b \\
(\mathrm{deg})\end{array}$ & $\begin{array}{l}\left\langle K_{\mathrm{s}}\right\rangle \\
(\mathrm{mag})\end{array}$ & $\begin{array}{c}P \\
\text { (days) }\end{array}$ & $\begin{array}{l}\text { Amplitude } \\
\text { (mag) }\end{array}$ & $\begin{array}{c}d \\
(\mathrm{kpc})\end{array}$ \\
\hline J181230.46-375218.9 & $18: 12: 30.46$ & $-37: 52: 18.9$ & -5.038 & -9.274 & 14.674 & 0.603464 & 0.34 & 11.6 \\
\hline J181230.83-395323.9 & $18: 12: 30.83$ & $-39: 53: 23.9$ & -6.860 & -10.196 & 13.954 & 0.652405 & 0.33 & 8.6 \\
\hline $\mathrm{J} 181237.48-350757.4 *$ & $18: 12: 37.48$ & $-35: 07: 57.4$ & -2.569 & -8.032 & 13.692 & 0.477544 & 0.35 & 6.4 \\
\hline $\mathrm{J} 181240.59-364330.2 *$ & $18: 12: 40.59$ & $-36: 43: 30.2$ & -3.991 & -8.777 & 14.586 & 0.445500 & 0.28 & 9.5 \\
\hline J181241.48-370833.3 & $18: 12: 41.48$ & $-37: 08: 33.3$ & -4.365 & -8.972 & 14.702 & 0.571893 & 0.27 & 11.5 \\
\hline J181245.83-375541.8 & $18: 12: 45.83$ & $-37: 55: 41.8$ & -5.066 & -9.345 & 14.875 & 0.606211 & 0.25 & 12.9 \\
\hline $\mathrm{J} 181246.22-352457.1 *$ & $18: 12: 46.22$ & $-35: 24: 57.1$ & -2.808 & -8.190 & 12.353 & 0.524444 & 0.32 & 3.5 \\
\hline J181247.62-391702.5 & $18: 12: 47.62$ & $-39: 17: 02.5$ & -6.287 & -9.969 & 14.530 & 0.564468 & 0.29 & 10.5 \\
\hline J181247.74-390544.2 & $18: 12: 47.74$ & $-39: 05: 44.2$ & -6.116 & -9.883 & 13.646 & 0.531912 & 0.32 & 6.6 \\
\hline $\mathrm{J} 181250.96-362307.2$ & $18: 12: 50.96$ & $-36: 23: 07.2$ & -3.670 & -8.652 & 14.130 & 0.602519 & 0.22 & 8.9 \\
\hline J181251.10-393101.4 & $18: 12: 51.10$ & $-39: 31: 01.4$ & -6.493 & -10.084 & 14.166 & 0.917979 & 0.19 & 11.4 \\
\hline J181251.43-384629.8 & $18: 12: 51.43$ & $-38: 46: 29.8$ & -5.821 & -9.748 & 14.369 & 0.629426 & 0.24 & 10.3 \\
\hline J181251.85-383309.2 & $18: 12: 51.85$ & $-38: 33: 09.2$ & -5.620 & -9.648 & 13.940 & 0.764368 & 0.23 & 9.3 \\
\hline J181252.56-383242.3 & $18: 12: 52.56$ & $-38: 32: 42.3$ & -5.612 & -9.646 & 14.293 & 0.557476 & 0.28 & 9.3 \\
\hline J181256.59-381912.2 & $18: 12: 56.59$ & $-38: 19: 12.2$ & -5.403 & -9.556 & 13.905 & 0.591478 & 0.22 & 7.9 \\
\hline $\mathrm{J} 181300.08-362149.5 *$ & 18:13:00.08 & $-36: 21: 49.5$ & -3.636 & -8.669 & 14.132 & 0.523733 & 0.32 & 8.3 \\
\hline J181300.84-353924.8 & $18: 13: 00.84$ & $-35: 39: 24.8$ & -3.001 & -8.345 & 15.313 & 0.638574 & 0.26 & 16.3 \\
\hline J181301.40-375149.8 & $18: 13: 01.40$ & $-37: 51: 49.8$ & -4.984 & -9.361 & 13.671 & 0.526518 & 0.24 & 6.7 \\
\hline J181306.46-373238.6 & 18:13:06.46 & $-37: 32: 38.6$ & -4.688 & -9.230 & 13.808 & 0.532229 & 0.28 & 7.1 \\
\hline $\mathrm{J} 181306.57-354528.3 *$ & $18: 13: 06.57$ & $-35: 45: 28.3$ & -3.083 & -8.409 & 13.955 & 0.594375 & 0.26 & 8.1 \\
\hline J181307.24-373449.5 & 18:13:07.24 & $-37: 34: 49.5$ & -4.720 & -9.249 & 14.496 & 0.509359 & 0.21 & 9.7 \\
\hline J181309.06-364700.2 & 18:13:09.06 & $-36: 47: 00.2$ & -4.000 & -8.889 & 15.360 & 0.588263 & 0.23 & 16.0 \\
\hline J181309.33-372501.6 & 18:13:09.33 & $-37: 25: 01.6$ & -4.569 & -9.180 & 14.342 & 0.504370 & 0.31 & 9.0 \\
\hline J181316.33-380958.9 & $18: 13: 16.33$ & $-38: 09: 58.9$ & -5.234 & -9.543 & 14.121 & 0.630728 & 0.27 & 9.1 \\
\hline $\mathrm{J} 181330.63-354714.7 *$ & $18: 13: 30.63$ & $-35: 47: 14.7$ & -3.071 & -8.495 & 13.615 & 0.825110 & 0.30 & 8.3 \\
\hline J181331.48-373248.5 & $18: 13: 31.48$ & $-37: 32: 48.5$ & -4.652 & -9.304 & 14.344 & 0.687673 & 0.24 & 10.6 \\
\hline J181333.94-362436.3 & 18:13:33.94 & $-36: 24: 36.3$ & -3.625 & -8.791 & 13.767 & 0.615684 & 0.46 & 7.6 \\
\hline J181335.48-390257.1 & $18: 13: 35.48$ & $-39: 02: 57.1$ & -6.003 & -10.000 & 14.505 & 0.556632 & 0.31 & 10.3 \\
\hline $\mathrm{J} 181345.63-375455.2 *$ & $18: 13: 45.63$ & $-37: 54: 55.2$ & -4.963 & -9.514 & 13.761 & 0.413310 & 0.29 & 6.1 \\
\hline J181349.89-392931.4* & $18: 13: 49.89$ & $-39: 29: 31.4$ & -6.383 & -10.242 & 14.052 & 0.601830 & 0.25 & 8.6 \\
\hline J181350.68-390037.6 & $18: 13: 50.68$ & $-39: 00: 37.6$ & -5.946 & -10.026 & 14.557 & 0.522463 & 0.28 & 10.2 \\
\hline $\mathrm{J} 181352.23-352030.4$ & $18: 13: 52.23$ & $-35: 20: 30.4$ & -2.637 & -8.354 & 14.073 & 0.504500 & 0.23 & 7.9 \\
\hline $\mathrm{J} 181352.62-361430.8 *$ & $18: 13: 52.62$ & $-36: 14: 30.8$ & -3.445 & -8.770 & 13.919 & 0.444735 & 0.34 & 6.8 \\
\hline J181356.78-373218.7 & $18: 13: 56.78$ & $-37: 32: 18.7$ & -4.606 & -9.375 & 12.964 & 0.650030 & 0.30 & 5.3 \\
\hline J181357.32-351854.9 & $18: 13: 57.32$ & $-35: 18: 54.9$ & -2.605 & -8.358 & 14.311 & 0.754245 & 0.27 & 11.0 \\
\hline $\mathrm{J} 181359.06-363846.1 *$ & $18: 13: 59.06$ & $-36: 38: 46.1$ & -3.799 & -8.974 & 13.793 & 0.470396 & 0.36 & 6.6 \\
\hline J181402.22-372154.2 & $18: 14: 02.22$ & $-37: 21: 54.2$ & -4.441 & -9.312 & 14.236 & 0.449275 & 0.30 & 8.0 \\
\hline $\mathrm{J} 181402.44-363624.9 *$ & $18: 14: 02.44$ & $-36: 36: 24.9$ & -3.758 & -8.966 & 14.009 & 0.582086 & 0.27 & 8.3 \\
\hline $\mathrm{J} 181403.26-380355.7 *$ & $18: 14: 03.26$ & $-38: 03: 55.7$ & -5.072 & -9.634 & 13.499 & 0.659326 & 0.35 & 6.9 \\
\hline J181403.91-354833.5 & 18:14:03.91 & $-35: 48: 33.5$ & -3.039 & -8.605 & 14.767 & 0.511535 & 0.33 & 11.1 \\
\hline J181405.00-371112.5 & $18: 14: 05.00$ & $-37: 11: 12.5$ & -4.276 & -9.239 & 14.079 & 0.512049 & 0.36 & 8.0 \\
\hline J181407.27-353413.8 & $18: 14: 07.27$ & $-35: 34: 13.8$ & -2.819 & -8.505 & 14.124 & 0.585514 & 0.29 & 8.8 \\
\hline J181411.54-354613.3 & $18: 14: 11.54$ & $-35: 46: 13.3$ & -2.992 & -8.610 & 13.983 & 0.651279 & 0.31 & 8.7 \\
\hline J181412.23-384017.6 & $18: 14: 12.23$ & $-38: 40: 17.6$ & -5.607 & -9.935 & 13.996 & 0.882535 & 0.17 & 10.3 \\
\hline J181415.16-345714.6* & $18: 14: 15.16$ & $-34: 57: 14.6$ & -2.253 & -8.245 & 13.738 & 0.567183 & 0.29 & 7.2 \\
\hline J181416.91-360644.7 & $18: 14: 16.91$ & $-36: 06: 44.7$ & -3.291 & -8.783 & 14.110 & 0.570363 & 0.25 & 8.6 \\
\hline J181418.46-375722.1 & $18: 14: 18.46$ & $-37: 57: 22.1$ & -4.950 & -9.629 & 14.210 & 0.542868 & 0.32 & 8.8 \\
\hline $\mathrm{J} 181418.96-352242.8 *$ & 18:14:18.96 & $-35: 22: 42.8$ & -2.628 & -8.452 & 14.046 & 0.396421 & 0.21 & 6.8 \\
\hline $\mathrm{J} 181426.56-351449.5 *$ & $18: 14: 26.56$ & $-35: 14: 49.5$ & -2.498 & -8.414 & 14.454 & 0.455158 & 0.35 & 9.0 \\
\hline J181429.19-380014.5 & 18:14:29.19 & $-38: 00: 14.5$ & -4.977 & -9.682 & 15.484 & 0.562430 & 0.26 & 16.6 \\
\hline J181429.52-391731.0 & $18: 14: 29.52$ & $-39: 17: 31.0$ & -6.143 & -10.265 & 14.380 & 0.596969 & 0.28 & 10.0 \\
\hline J181429.77-384405.0 & $18: 14: 29.77$ & $-38: 44: 05.0$ & -5.638 & -10.014 & 14.049 & 0.807422 & 0.24 & 10.1 \\
\hline J181430.58-352336.0 & $18: 14: 30.58$ & $-35: 23: 36.0$ & -2.623 & -8.494 & 14.279 & 0.547151 & 0.32 & 9.1 \\
\hline $\mathrm{J} 181433.66-355455.0 *$ & $18: 14: 33.66$ & $-35: 54: 55.0$ & -3.087 & -8.743 & 14.194 & 0.508839 & 0.25 & 8.4 \\
\hline $\mathrm{J} 181434.68-345906.3 *$ & $18: 14: 34.68$ & $-34: 59: 06.3$ & -2.250 & -8.319 & 14.686 & 0.489179 & 0.24 & 10.4 \\
\hline $\mathrm{J} 181442.25-363044.3$ & $18: 14: 42.25$ & $-36: 30: 44.3$ & -3.611 & -9.041 & 14.689 & 0.487439 & 0.30 & 10.4 \\
\hline J181444.37-373821.4 & $18: 14: 44.37$ & $-37: 38: 21.4$ & -4.625 & -9.561 & 14.652 & 0.474841 & 0.39 & 10.1 \\
\hline $\mathrm{J} 181445.81-352035.6 *$ & $18: 14: 45.81$ & $-35: 20: 35.6$ & -2.554 & -8.517 & 13.676 & 0.609567 & 0.35 & 7.2 \\
\hline $\mathrm{J} 181445.97-362705.8 *$ & $18: 14: 45.97$ & $-36: 27: 05.8$ & -3.551 & -9.025 & 14.378 & 0.474532 & 0.34 & 8.9 \\
\hline J181446.70-352456.3 & $18: 14: 46.70$ & $-35: 24: 56.3$ & -2.618 & -8.553 & 14.037 & 0.546305 & 0.25 & 8.1 \\
\hline
\end{tabular}


Table A.1. continued.

\begin{tabular}{|c|c|c|c|c|c|c|c|c|}
\hline VVV ID & $\begin{array}{c}\text { RA (J2000.0) } \\
\text { hh:mm:ss.ss }\end{array}$ & $\begin{array}{c}\text { Dec (J2000.0) } \\
\text { dd:mm:ss.s }\end{array}$ & $\begin{array}{c}\ell \\
(\mathrm{deg}) \\
\end{array}$ & $\begin{array}{c}b \\
(\mathrm{deg}) \\
\end{array}$ & $\begin{array}{l}\left\langle K_{\mathrm{s}}\right\rangle \\
(\mathrm{mag}) \\
\end{array}$ & $\begin{array}{c}P \\
\text { (days) } \\
\end{array}$ & $\begin{array}{l}\text { Amplitude } \\
\text { (mag) }\end{array}$ & $\begin{array}{c}d \\
(\mathrm{kpc})\end{array}$ \\
\hline J181447.56-374749.2 & $18: 14: 47.56$ & $-37: 47: 49.2$ & -4.762 & -9.642 & 13.129 & 0.561060 & 0.28 & 5.3 \\
\hline J181458.41-351324.8* & 18:14:58.41 & $-35: 13: 24.8$ & -2.427 & -8.500 & 14.389 & 0.520159 & 0.30 & 9.4 \\
\hline J181505.11-374522.4 & 18:15:05.11 & $-37: 45: 22.4$ & -4.699 & -9.675 & 14.087 & 0.781305 & 0.32 & 10.1 \\
\hline J181505.80-390448.3 & 18:15:05.80 & $-39: 04: 48.3$ & -5.897 & -10.274 & 14.016 & 0.644749 & 0.32 & 8.8 \\
\hline $\mathrm{J} 181506.49-345411.4 *$ & 18:15:06.49 & $-34: 54: 11.4$ & -2.126 & -8.377 & 15.189 & 0.536993 & 0.30 & 14.0 \\
\hline J181507.09-382029.1 & 18:15:07.09 & $-38: 20: 29.1$ & -5.226 & -9.945 & 13.714 & 0.620582 & 0.28 & 7.4 \\
\hline J181507.57-350118.7 & $18: 15: 07.57$ & $-35: 01: 18.7$ & -2.231 & -8.435 & 14.615 & 0.566248 & 0.32 & 10.9 \\
\hline $\mathrm{J} 181507.97-364323.7 *$ & 18:15:07.97 & $-36: 43: 23.7$ & -3.762 & -9.214 & 14.142 & 0.546263 & 0.27 & 8.5 \\
\hline $\mathrm{J} 181510.34-382330.9 *$ & $18: 15: 10.34$ & $-38: 23: 30.9$ & -5.266 & -9.978 & 14.586 & 0.456704 & 0.37 & 9.6 \\
\hline J181519.07-341436.7 & $18: 15: 19.07$ & $-34: 14: 36.7$ & -1.514 & -8.112 & 14.675 & 0.475048 & 0.31 & 10.2 \\
\hline $\mathrm{J} 181519.98-365402.8 *$ & 18:15:19.98 & $-36: 54: 02.8$ & -3.903 & -9.331 & 13.821 & 0.452614 & 0.38 & 6.6 \\
\hline $\mathrm{J} 181521.94-344201.2 *$ & $18: 15: 21.94$ & $-34: 42: 01.2$ & -1.919 & -8.331 & 14.032 & 0.549354 & 0.28 & 8.1 \\
\hline J181522.33-354724.8 & $18: 15: 22.33$ & $-35: 47: 24.8$ & -2.899 & -8.832 & 14.273 & 0.589477 & 0.25 & 9.5 \\
\hline J181523.03-382850.0 & $18: 15: 23.03$ & $-38: 28: 50.0$ & -5.328 & -10.055 & 13.821 & 0.480247 & 0.32 & 6.8 \\
\hline J181525.60-384533.4 & $18: 15: 25.60$ & $-38: 45: 33.4$ & -5.577 & -10.187 & 13.583 & 0.612329 & 0.22 & 6.9 \\
\hline J181529.19-360538.7* & $18: 15: 29.19$ & $-36: 05: 38.7$ & -3.162 & -8.991 & 13.800 & 0.617086 & 0.33 & 7.7 \\
\hline J181529.48-352734.5 & $18: 15: 29.48$ & $-35: 27: 34.5$ & -2.590 & -8.702 & 13.871 & 0.918999 & 0.22 & 9.9 \\
\hline J181530.80-380609.8 & $18: 15: 30.80$ & $-38: 06: 09.8$ & -4.974 & -9.907 & 14.409 & 0.790170 & 0.32 & 11.8 \\
\hline J181531.81-360828.3 & $18: 15: 31.81$ & $-36: 08: 28.3$ & -3.200 & -9.020 & 14.499 & 0.527757 & 0.31 & 9.9 \\
\hline J181534.62-354126.4 & $18: 15: 34.62$ & $-35: 41: 26.4$ & -2.790 & -8.823 & 13.744 & 0.542023 & 0.26 & 7.0 \\
\hline J181535.05-344202.4* & $18: 15: 35.05$ & $-34: 42: 02.4$ & -1.899 & -8.371 & 14.085 & 0.483075 & 0.37 & 7.8 \\
\hline J181544.14-372806.6* & $18: 15: 44.14$ & $-37: 28: 06.6$ & -4.379 & -9.659 & 12.953 & 0.555133 & 0.27 & 4.8 \\
\hline J181554.37-374658.5 & $18: 15: 54.37$ & $-37: 46: 58.5$ & -4.649 & -9.832 & 14.085 & 0.573895 & 0.26 & 8.5 \\
\hline J181556.68-341822.9 & $18: 15: 56.68$ & $-34: 18: 22.9$ & -1.510 & -8.256 & 15.353 & 0.553934 & 0.29 & 15.4 \\
\hline $\mathrm{J} 181558.80-352754.8 *$ & $18: 15: 58.80$ & $-35: 27: 54.8$ & -2.549 & -8.793 & 14.750 & 0.532122 & 0.29 & 11.3 \\
\hline J181558.88-344456.9 & $18: 15: 58.88$ & $-34: 44: 56.9$ & -1.905 & -8.466 & 13.500 & 0.553085 & 0.29 & 6.3 \\
\hline J181605.98-382259.4* & 18:16:05.98 & $-38: 22: 59.4$ & -5.175 & -10.136 & 13.705 & 0.679648 & 0.26 & 7.8 \\
\hline J181610.53-342233.3 & $18: 16: 10.53$ & $-34: 22: 33.3$ & -1.551 & -8.331 & 14.042 & 0.651988 & 0.28 & 8.9 \\
\hline $\mathrm{J} 181614.93-364712.9 *$ & $18: 16: 14.93$ & $-36: 47: 12.9$ & -3.716 & -9.442 & 15.289 & 0.441985 & 0.38 & 13.2 \\
\hline $\mathrm{J} 181617.59-365626.5 *$ & 18:16:17.59 & $-36: 56: 26.5$ & -3.851 & -9.520 & 13.631 & 0.634192 & 0.26 & 7.2 \\
\hline $\mathrm{J} 181619.68-353803.8 *$ & 18:16:19.68 & $-35: 38: 03.8$ & -2.669 & -8.933 & 13.268 & 0.474672 & 0.30 & 5.2 \\
\hline J181620.17-363931.6 & 18:16:20.17 & $-36: 39: 31.6$ & -3.592 & -9.400 & 14.135 & 0.596819 & 0.25 & 8.9 \\
\hline J181622.34-360017.3 & $18: 16: 22.34$ & $-36: 00: 17.3$ & -2.998 & -9.110 & 14.368 & 0.605301 & 0.21 & 10.0 \\
\hline J181624.45-341312.6 & $18: 16: 24.45$ & $-34: 13: 12.6$ & -1.389 & -8.302 & 15.166 & 0.488091 & 0.35 & 13.2 \\
\hline $\mathrm{J} 181629.04-351629.9 *$ & 18:16:29.04 & $-35: 16: 29.9$ & -2.330 & -8.798 & 13.740 & 0.681566 & 0.30 & 7.9 \\
\hline J181629.52-352507.5* & $18: 16: 29.52$ & $-35: 25: 07.5$ & -2.459 & -8.865 & 13.475 & 0.512702 & 0.32 & 6.0 \\
\hline J181631.23-351726.8* & $18: 16: 31.23$ & $-35: 17: 26.8$ & -2.341 & -8.812 & 13.904 & 0.481900 & 0.32 & 7.1 \\
\hline $\mathrm{J} 181632.17-334319.8 * *$ & $18: 16: 32.17$ & $-33: 43: 19.8$ & -0.929 & -8.097 & 14.315 & 0.547981 & 0.32 & 9.3 \\
\hline $\mathrm{J} 181632.30-334141.8 *$ & $18: 16: 32.30$ & $-33: 41: 41.8$ & -0.904 & -8.085 & 14.015 & 0.499880 & 0.32 & 7.6 \\
\hline J181634.05-362901.3 & $18: 16: 34.05$ & $-36: 29: 01.3$ & -3.413 & -9.362 & 15.820 & 0.434575 & 0.29 & 17.0 \\
\hline $\mathrm{J} 181640.87-354614.0 *$ & $18: 16: 40.87$ & $-35: 46: 14.0$ & -2.758 & -9.059 & 13.964 & 0.657761 & 0.31 & 8.6 \\
\hline $\mathrm{J} 181643.25-340122.3 *$ & $18: 16: 43.25$ & $-34: 01: 22.3$ & -1.181 & -8.269 & 14.394 & 0.605631 & 0.35 & 10.2 \\
\hline J181644.94-375311.3 & $18: 16: 44.94$ & $-37: 53: 11.3$ & -4.666 & -10.027 & 14.225 & 0.529928 & 0.29 & 8.7 \\
\hline $\mathrm{J} 181645.55-333359.5 *$ & $18: 16: 45.55$ & $-33: 33: 59.5$ & -0.768 & -8.067 & 14.310 & 0.473458 & 0.32 & 8.6 \\
\hline J181645.58-335150.3 & $18: 16: 45.58$ & $-33: 51: 50.3$ & -1.035 & -8.203 & 14.517 & 0.532439 & 0.32 & 10.1 \\
\hline $\mathrm{J} 181649.66-352017.7 *$ & $18: 16: 49.66$ & $-35: 20: 17.7$ & -2.355 & -8.889 & 13.979 & 0.497953 & 0.34 & 7.5 \\
\hline J181650.07-342516.7 & $18: 16: 50.07$ & $-34: 25: 16.7$ & -1.529 & -8.472 & 14.601 & 0.503398 & 0.29 & 10.2 \\
\hline J181652.16-344627.5 & 18:16:52.16 & $-34: 46: 27.5$ & -1.843 & -8.640 & 13.435 & 0.575245 & 0.26 & 6.2 \\
\hline $\mathrm{J} 181652.89-375727.9 *$ & 18:16:52.89 & $-37: 57: 27.9$ & -4.719 & -10.082 & 13.407 & 0.488081 & 0.34 & 5.6 \\
\hline J181654.67-343116.3 & $18: 16: 54.67$ & $-34: 31: 16.3$ & -1.611 & -8.532 & 15.224 & 0.445230 & 0.41 & 12.9 \\
\hline $\mathrm{J} 181657.56-381809.1 *$ & 18:16:57.56 & $-38: 18: 09.1$ & -5.025 & -10.250 & 13.851 & 0.656495 & 0.28 & 8.2 \\
\hline J181657.76-353708.8 & 18:16:57.76 & $-35: 37: 08.8$ & -2.595 & -9.041 & 14.274 & 0.583475 & 0.23 & 9.4 \\
\hline J181658.01-342536.1 & 18:16:58.01 & $-34: 25: 36.1$ & -1.521 & -8.499 & 14.354 & 0.560834 & 0.27 & 9.6 \\
\hline $\mathrm{J} 181700.49-341727.9 *$ & 18:17:00.49 & $-34: 17: 27.9$ & -1.395 & -8.445 & 14.650 & 0.453256 & 0.36 & 9.8 \\
\hline J181704.07-355439.0 & 18:17:04.07 & $-35: 54: 39.0$ & -2.849 & -9.192 & 14.660 & 0.540368 & 0.30 & 10.9 \\
\hline J181704.56-375124.0 & 18:17:04.56 & $-37: 51: 24.0$ & -4.610 & -10.071 & 14.652 & 0.494868 & 0.26 & 10.3 \\
\hline J181705.48-331940.9 & 18:17:05.48 & $-33: 19: 40.9$ & -0.522 & -8.019 & 13.591 & 0.553302 & 0.32 & 6.6 \\
\hline $\mathrm{J} 181706.67-341613.8 *$ & $18: 17: 06.67$ & $-34: 16: 13.8$ & -1.367 & -8.454 & 13.919 & 0.684266 & 0.26 & 8.6 \\
\hline J181709.51-344730.4 & 18:17:09.51 & $-34: 47: 30.4$ & -1.831 & -8.700 & 14.595 & 0.497045 & 0.25 & 10.1 \\
\hline J181711.65-365037.6 & $18: 17: 11.65$ & $-36: 50: 37.6$ & -3.681 & -9.637 & 14.782 & 0.458781 & 0.37 & 10.6 \\
\hline
\end{tabular}


Table A.1. continued.

\begin{tabular}{|c|c|c|c|c|c|c|c|c|}
\hline VVV ID & $\begin{array}{c}\text { RA (J2000.0) } \\
\text { hh:mm:ss.ss }\end{array}$ & $\begin{array}{c}\text { Dec (J2000.0) } \\
\text { dd:mm:ss.s }\end{array}$ & $\begin{array}{c}\ell \\
\text { (deg) }\end{array}$ & $\begin{array}{c}b \\
(\mathrm{deg})\end{array}$ & $\begin{array}{l}\left\langle K_{\mathrm{s}}\right\rangle \\
(\mathrm{mag})\end{array}$ & $\begin{array}{c}P \\
\text { (days) }\end{array}$ & $\begin{array}{l}\text { Amplitude } \\
\text { (mag) }\end{array}$ & $\begin{array}{c}d \\
(\mathrm{kpc})\end{array}$ \\
\hline J181713.34-372529.4 & 18:17:13.34 & $-37: 25: 29.4$ & -4.205 & -9.903 & 14.074 & 0.611086 & 0.16 & 8.8 \\
\hline J181718.08-375812.6 & $18: 17: 18.08$ & $-37: 58: 12.6$ & -4.693 & -10.162 & 14.277 & 0.577264 & 0.33 & 9.4 \\
\hline $\mathrm{J} 181723.79-371424.2$ & $18: 17: 23.79$ & $-37: 14: 24.2$ & -4.022 & -9.851 & 14.357 & 0.520143 & 0.32 & 9.2 \\
\hline J181724.11-371520.9 & $18: 17: 24.11$ & $-37: 15: 20.9$ & -4.035 & -9.859 & 14.776 & 0.556967 & 0.29 & 11.7 \\
\hline J181724.66-345352.6 & $18: 17: 24.66$ & $-34: 53: 52.6$ & -1.903 & -8.795 & 14.332 & 0.462888 & 0.29 & 8.5 \\
\hline $\mathrm{J} 181725.42-334742.4 *$ & $18: 17: 25.42$ & $-33: 47: 42.4$ & -0.909 & -8.295 & 13.950 & 0.534068 & 0.20 & 7.7 \\
\hline $\mathrm{J} 181726.05-361312.1 *$ & $18: 17: 26.05$ & $-36: 13: 12.1$ & -3.094 & -9.398 & 13.709 & 0.707879 & 0.30 & 7.9 \\
\hline $\mathrm{J} 181727.29-335532.1 * *$ & $18: 17: 27.29$ & $-33: 55: 32.1$ & -1.024 & -8.360 & 13.612 & 0.675320 & 0.20 & 7.4 \\
\hline J181727.55-354403.3* & $18: 17: 27.55$ & $-35: 44: 03.3$ & -2.653 & -9.183 & 14.252 & 0.623970 & 0.30 & 9.7 \\
\hline $\mathrm{J} 181733.97-353109.9 *$ & $18: 17: 33.97$ & $-35: 31: 09.9$ & -2.449 & -9.105 & 14.094 & 0.602439 & 0.33 & 8.8 \\
\hline $\mathrm{J} 181742.23-344836.6 *$ & $18: 17: 42.23$ & $-34: 48: 36.6$ & -1.796 & -8.809 & 12.531 & 0.619138 & 0.35 & 4.2 \\
\hline $\mathrm{J} 181745.11-333025.2 * *$ & $18: 17: 45.11$ & $-33: 30: 25.2$ & -0.619 & -8.224 & 14.951 & 0.505283 & 0.30 & 12.1 \\
\hline $\mathrm{J} 181745.70-333214.2 *$ & $18: 17: 45.70$ & $-33: 32: 14.2$ & -0.645 & -8.239 & 14.915 & 0.440511 & 0.24 & 11.0 \\
\hline $\mathrm{J} 181746.66-335654.5 *$ & $18: 17: 46.66$ & $-33: 56: 54.5$ & -1.013 & -8.430 & 13.849 & 0.567769 & 0.27 & 7.5 \\
\hline J181747.66-342504.7 & $18: 17: 47.66$ & $-34: 25: 04.7$ & -1.434 & -8.647 & 14.181 & 0.623088 & 0.31 & 9.3 \\
\hline $\mathrm{J} 181749.89-335146.2 *$ & $18: 17: 49.89$ & $-33: 51: 46.2$ & -0.931 & -8.401 & 14.726 & 0.686168 & 0.31 & 12.8 \\
\hline J181749.93-344116.0 & $18: 17: 49.93$ & $-34: 41: 16.0$ & -1.674 & -8.776 & 14.168 & 0.531098 & 0.24 & 8.5 \\
\hline $\mathrm{J} 181750.90-360357.6 *$ & 18:17:50.90 & $-36: 03: 57.6$ & -2.917 & -9.403 & 15.054 & 0.405131 & 0.30 & 11.3 \\
\hline $\mathrm{J} 181752.16-372251.6 *$ & $18: 17: 52.16$ & $-37: 22: 51.6$ & -4.107 & -9.998 & 14.290 & 0.450187 & 0.27 & 8.2 \\
\hline $\mathrm{J} 181752.57-354131.3$ & $18: 17: 52.57$ & $-35: 41: 31.3$ & -2.576 & -9.240 & 15.398 & 0.452464 & 0.40 & 14.1 \\
\hline J181752.60-331543.8 & $18: 17: 52.60$ & $-33: 15: 43.8$ & -0.387 & -8.135 & 14.912 & 0.468960 & 0.24 & 11.4 \\
\hline J181802.62-374619.9 & 18:18:02.62 & $-37: 46: 19.9$ & -4.446 & -10.204 & 14.031 & 0.552614 & 0.28 & 8.1 \\
\hline J181805.35-325841.8 & 18:18:05.35 & $-32: 58: 41.8$ & -0.111 & -8.045 & 14.522 & 0.593349 & 0.41 & 10.7 \\
\hline $\mathrm{J} 181805.66-363810.4 *$ & 18:18:05.66 & $-36: 38: 10.4$ & -3.411 & -9.704 & 14.522 & 0.574538 & 0.28 & 10.5 \\
\hline J181806.34-370306.8 & 18:18:06.34 & $-37: 03: 06.8$ & -3.787 & -9.893 & 13.694 & 0.596986 & 0.26 & 7.2 \\
\hline J181807.82-361359.6* & 18:18:07.82 & $-36: 13: 59.6$ & -3.042 & -9.530 & 13.803 & 0.452860 & 0.38 & 6.5 \\
\hline $\mathrm{J} 181808.39-341844.2 *$ & 18:18:08.39 & $-34: 18: 44.2$ & -1.306 & -8.662 & 13.006 & 0.643832 & 0.33 & 5.4 \\
\hline J181809.19-352945.0 & 18:18:09.19 & $-35: 29: 45.0$ & -2.373 & -9.201 & 14.424 & 0.569671 & 0.31 & 10.0 \\
\hline J181810.15-373707.4 & 18:18:10.15 & $-37: 37: 07.4$ & -4.296 & -10.158 & 14.581 & 0.571327 & 0.31 & 10.8 \\
\hline $\mathrm{J} 181811.59-350633.2 *$ & 18:18:11.59 & $-35: 06: 33.2$ & -2.020 & -9.034 & 14.707 & 0.473711 & 0.34 & 10.4 \\
\hline $\mathrm{J} 181812.84-351121.0$ & $18: 18: 12.84$ & $-35: 11: 21.0$ & -2.090 & -9.073 & 13.648 & 0.615635 & 0.20 & 7.2 \\
\hline J181812.89-372939.2 & 18:18:12.89 & $-37: 29: 39.2$ & -4.178 & -10.110 & 15.892 & 0.554961 & 0.34 & 20.0 \\
\hline $\mathrm{J} 181814.52-325645.4 *$ & $18: 18: 14.52$ & $-32: 56: 45.4$ & -0.067 & -8.059 & 14.226 & 0.464313 & 0.28 & 8.1 \\
\hline $\mathrm{J} 181817.14-361754.9 *$ & $18: 18: 17.14$ & $-36: 17: 54.9$ & -3.087 & -9.587 & 14.724 & 0.480716 & 0.33 & 10.5 \\
\hline J181819.94-363905.2 & 18:18:19.94 & $-36: 39: 05.2$ & -3.403 & -9.754 & 13.326 & 0.656439 & 0.25 & 6.3 \\
\hline J181820.31-363721.3 & 18:18:20.31 & $-36: 37: 21.3$ & -3.376 & -9.742 & 13.853 & 0.693900 & 0.25 & 8.4 \\
\hline $\mathrm{J} 181824.70-363713.1 *$ & $18: 18: 24.70$ & $-36: 37: 13.1$ & -3.367 & -9.754 & 13.962 & 0.619276 & 0.34 & 8.4 \\
\hline $\mathrm{J} 181825.62-374652.8$ & $18: 18: 25.62$ & $-37: 46: 52.8$ & -4.420 & -10.276 & 13.132 & 0.509510 & 0.30 & 5.0 \\
\hline J181826.01-363824.1 & $18: 18: 26.01$ & $-36: 38: 24.1$ & -3.383 & -9.767 & 13.506 & 0.607478 & 0.20 & 6.6 \\
\hline J181829.96-335611.7 & 18:18:29.96 & $-33: 56: 11.7$ & -0.934 & -8.558 & 14.001 & 0.616835 & 0.27 & 8.5 \\
\hline J181831.18-341728.6 & 18:18:31.18 & $-34: 17: 28.6$ & -1.251 & -8.723 & 13.866 & 0.565563 & 0.25 & 7.6 \\
\hline J181832.29-333757.2 & $18: 18: 32.29$ & $-33: 37: 57.2$ & -0.656 & -8.427 & 14.107 & 0.599797 & 0.27 & 8.8 \\
\hline $\mathrm{J} 181833.18-350456.0$ & $18: 18: 33.18$ & $-35: 04: 56.0$ & -1.962 & -9.087 & 13.805 & 0.527582 & 0.22 & 7.1 \\
\hline $\mathrm{J} 181838.33-351351.3 *$ & $18: 18: 38.33$ & $-35: 13: 51.3$ & -2.088 & -9.170 & 14.339 & 0.686243 & 0.32 & 10.6 \\
\hline J181843.83-335631.3 & $18: 18: 43.83$ & $-33: 56: 31.3$ & -0.917 & -8.603 & 13.477 & 0.570402 & 0.28 & 6.3 \\
\hline J181845.54-324431.1 & $18: 18: 45.54$ & $-32: 44: 31.1$ & 0.166 & -8.062 & 13.791 & 0.629378 & 0.22 & 7.8 \\
\hline J181851.35-330614.5 & $18: 18: 51.35$ & $-33: 06: 14.5$ & -0.150 & -8.245 & 13.260 & 0.516041 & 0.28 & 5.4 \\
\hline $\mathrm{J} 181852.52-344107.2 *$ & $18: 18: 52.52$ & $-34: 41: 07.2$ & -1.573 & -8.967 & 13.954 & 0.576515 & 0.26 & 8.0 \\
\hline $\mathrm{J} 181857.42-330033.9 *$ & 18:18:57.42 & $-33: 00: 33.9$ & -0.055 & -8.221 & 14.048 & 0.528505 & 0.29 & 8.0 \\
\hline J181903.62-351425.7 & 18:19:03.62 & $-35: 14: 25.7$ & -2.058 & -9.251 & 15.040 & 0.813829 & 0.19 & 16.3 \\
\hline J181908.93-335354.1* & 18:19:08.93 & $-33: 53: 54.1$ & -0.837 & -8.661 & 14.543 & 0.479322 & 0.32 & 9.6 \\
\hline J181910.10-331205.4 & 18:19:10.10 & $-33: 12: 05.4$ & -0.208 & -8.348 & 14.071 & 0.493905 & 0.25 & 7.8 \\
\hline J181910.91-330421.6* & 18:19:10.91 & $-33: 04: 21.6$ & -0.090 & -8.292 & 13.811 & 0.510326 & 0.31 & 7.0 \\
\hline J181913.13-353545.5 & 18:19:13.13 & $-35: 35: 45.5$ & -2.365 & -9.440 & 13.762 & 0.572931 & 0.27 & 7.3 \\
\hline $\mathrm{J} 181916.58-354855.4 *$ & 18:19:16.58 & $-35: 48: 55.4$ & -2.558 & -9.549 & 14.723 & 0.553814 & 0.38 & 11.4 \\
\hline $\mathrm{J} 181917.03-331351.5 *$ & $18: 19: 17.03$ & $-33: 13: 51.5$ & -0.223 & -8.383 & 14.682 & 0.468064 & 0.36 & 10.2 \\
\hline J181917.76-361647.4* & 18:19:17.76 & $-36: 16: 47.4$ & -2.977 & -9.760 & 13.537 & 0.607648 & 0.33 & 6.7 \\
\hline J181918.16-365404.2 & 18:19:18.16 & $-36: 54: 04.2$ & -3.541 & -10.039 & 14.325 & 0.490595 & 0.30 & 8.8 \\
\hline J181919.44-363429.4* & 18:19:19.44 & $-36: 34: 29.4$ & -3.243 & -9.897 & 14.314 & 0.496934 & 0.34 & 8.8 \\
\hline J181923.06-323720.0 & $18: 19: 23.06$ & $-32: 37: 20.0$ & 0.334 & -8.124 & 14.246 & 0.535957 & 0.30 & 8.9 \\
\hline
\end{tabular}


Table A.1. continued.

\begin{tabular}{|c|c|c|c|c|c|c|c|c|}
\hline VVV ID & $\begin{array}{l}\text { RA (J2000.0) } \\
\text { hh:mm:ss.ss }\end{array}$ & $\begin{array}{c}\text { Dec (J2000.0) } \\
\text { dd:mm:ss.s }\end{array}$ & $\begin{array}{c}\ell \\
(\mathrm{deg})\end{array}$ & $\begin{array}{c}b \\
(\mathrm{deg})\end{array}$ & $\begin{array}{l}\left\langle K_{\mathrm{s}}\right\rangle \\
(\mathrm{mag})\end{array}$ & $\begin{array}{c}P \\
\text { (days) }\end{array}$ & $\begin{array}{c}\text { Amplitude } \\
\text { (mag) }\end{array}$ & $\begin{array}{c}d \\
(\mathrm{kpc})\end{array}$ \\
\hline J181923.70-352208.4* & $18: 19: 23.70$ & $-35: 22: 08.4$ & -2.143 & -9.370 & 15.270 & 0.406951 & 0.34 & 12.5 \\
\hline J181926.21-362655.4 & 18:19:26.21 & $-36: 26: 55.4$ & -3.118 & -9.861 & 14.527 & 0.544556 & 0.23 & 10.2 \\
\hline $\mathrm{J} 181926.95-345327.4 *$ & $18: 19: 26.95$ & $-34: 53: 27.4$ & -1.705 & -9.164 & 13.466 & 0.601815 & 0.27 & 6.5 \\
\hline J181934.58-371913.7 & $18: 19: 34.58$ & $-37: 19: 13.7$ & -3.898 & -10.275 & 13.895 & 0.645403 & 0.24 & 8.3 \\
\hline J181934.72-355159.1 & $18: 19: 34.72$ & $-35: 51: 59.1$ & -2.577 & -9.626 & 13.798 & 0.589247 & 0.25 & 7.5 \\
\hline J181938.68-323528.0 & $18: 19: 38.68$ & $-32: 35: 28.0$ & 0.388 & -8.159 & 14.433 & 0.829071 & 0.21 & 12.3 \\
\hline J181940.69-352213.3 & $18: 19: 40.69$ & $-35: 22: 13.3$ & -2.118 & -9.422 & 15.299 & 0.583611 & 0.23 & 15.5 \\
\hline J181942.46-330138.6 & $18: 19: 42.46$ & $-33: 01: 38.6$ & 0.001 & -8.369 & 14.765 & 0.550170 & 0.32 & 11.6 \\
\hline J181942.60-325059.2 & $18: 19: 42.60$ & $-32: 50: 59.2$ & 0.161 & -8.289 & 13.790 & 0.626041 & 0.24 & 7.7 \\
\hline J181947.89-370911.6 & 18:19:47.89 & $-37: 09: 11.6$ & -3.726 & -10.24 & 14.417 & 0.483823 & 0.29 & 9.1 \\
\hline J181948.47-344444.2 & $18: 19: 48.47$ & $-34: 44: 44.2$ & -1.540 & -9.165 & 14.813 & 0.475213 & 0.29 & 10.9 \\
\hline J181949.50-333508.8 & $18: 19: 49.50$ & $-33: 35: 08.8$ & -0.491 & -8.644 & 15.14 & 0.501794 & 0.24 & 13.2 \\
\hline J181950.31-332444.7 & $18: 19: 50.31$ & $-33: 24: 44.7$ & -0.333 & -8.568 & 13.941 & 0.783883 & 0.21 & 9.4 \\
\hline J181954.41-324748.5 & $18: 19: 54.41$ & $-32: 47: 48.5$ & 0.228 & -8.302 & 14.032 & 0.559394 & 0.24 & 8.2 \\
\hline J182002.81-353303.4 & 18:20:02.81 & $-35: 33: 03.4$ & -2.247 & -9.570 & 14.753 & 0.538297 & 0.29 & 11.4 \\
\hline J182004.97-355809.5 & 18:20:04.97 & $-35: 58: 09.5$ & -2.624 & -9.764 & 14.456 & 0.534345 & 0.32 & 9.8 \\
\hline $\mathrm{J} 182007.65-344558.4 *$ & 18:20:07.65 & $-34: 45: 58.4$ & -1.529 & -9.233 & 14.702 & 0.489582 & 0.34 & 10.5 \\
\hline J182011.73-322258.3 & $18: 20: 11.73$ & $-32: 22: 58.3$ & 0.628 & -8.168 & 14.148 & 0.511687 & 0.33 & 8.2 \\
\hline $\mathrm{J} 182012.03-354837.4 *$ & $18: 20: 12.03$ & $-35: 48: 37.4$ & -2.468 & -9.714 & 13.057 & 0.545292 & 0.29 & 5.0 \\
\hline J182017.11-344434.0 & 18:20:17.11 & $-34: 44: 34.0$ & -1.493 & -9.251 & 14.220 & 0.589237 & 0.27 & 9.2 \\
\hline $\mathrm{J} 182017.52-352722.1 *$ & $18: 20: 17.52$ & $-35: 27: 22.1$ & -2.139 & -9.572 & 13.821 & 0.591975 & 0.33 & 7.6 \\
\hline J182022.62-330607.9 & $18: 20: 22.62$ & $-33: 06: 07.9$ & -0.002 & -8.528 & 14.429 & 0.468187 & 0.33 & 9.0 \\
\hline J182030.40-325128.9 & $18: 20: 30.40$ & $-32: 51: 28.9$ & 0.230 & -8.442 & 14.199 & 0.759065 & 0.27 & 10.5 \\
\hline J182030.62-345803.8 & $18: 20: 30.62$ & $-34: 58: 03.8$ & -1.676 & -9.393 & 14.540 & 0.600759 & 0.26 & 10.9 \\
\hline J182033.23-315746.5 & $18: 20: 33.23$ & $-31: 57: 46.5$ & 1.041 & -8.045 & 14.171 & 0.626060 & 0.22 & 9.3 \\
\hline J182035.35-322632.4 & $18: 20: 35.35$ & $-32: 26: 32.4$ & 0.613 & -8.269 & 14.489 & 0.480705 & 0.35 & 9.4 \\
\hline $\mathrm{J} 182037.48-335458.0 *$ & $18: 20: 37.48$ & $-33: 54: 58.0$ & -0.713 & -8.942 & 14.004 & 0.435692 & 0.36 & 7.1 \\
\hline $\mathrm{J} 182038.80-341525.6 *$ & $18: 20: 38.80$ & $-34: 15: 25.6$ & -1.020 & -9.099 & 14.194 & 0.605451 & 0.26 & 9.2 \\
\hline J182043.49-361309.0 & $18: 20: 43.49$ & $-36: 13: 09.0$ & -2.792 & -9.991 & 14.353 & 0.467854 & 0.37 & 8.7 \\
\hline J182044.58-352919.2 & $18: 20: 44.58$ & $-35: 29: 19.2$ & -2.127 & -9.669 & 15.066 & 0.503593 & 0.27 & 12.7 \\
\hline $\mathrm{J} 182053.23-355135.9 *$ & $18: 20: 53.23$ & $-35: 51: 35.9$ & -2.451 & -9.861 & 13.992 & 0.531898 & 0.22 & 7.8 \\
\hline $\mathrm{J} 182055.80-335539.8 *$ & $18: 20: 55.80$ & $-33: 55: 39.8$ & -0.695 & -9.004 & 14.122 & 0.476669 & 0.35 & 7.8 \\
\hline J182055.93-342729.1 & $18: 20: 55.93$ & $-34: 27: 29.1$ & -1.175 & -9.242 & 14.522 & 0.599481 & 0.31 & 10.8 \\
\hline J182058.26-333521.6 & $18: 20: 58.26$ & $-33: 35: 21.6$ & -0.385 & -8.859 & 14.811 & 0.544324 & 0.26 & 11.8 \\
\hline $\mathrm{J} 182059.72-334555.2$ & $18: 20: 59.72$ & $-33: 45: 55.2$ & -0.542 & -8.943 & 14.385 & 0.624859 & 0.32 & 10.3 \\
\hline J182059.77-333350.6 & $18: 20: 59.77$ & $-33: 33: 50.6$ & -0.360 & -8.852 & 13.533 & 0.652857 & 0.25 & 7.0 \\
\hline J182100.49-330417.3 & 18:21:00.49 & $-33: 04: 17.3$ & 0.086 & -8.632 & 14.370 & 0.623873 & 0.23 & 10.2 \\
\hline $\mathrm{J} 182102.18-334829.5 *$ & :02.18 & $-33: 48: 29.5$ & -0.577 & -8.969 & 13.285 & 0.534502 & 0.29 & 5.6 \\
\hline J182106.50-314952.8 & $21: 06.50$ & $-31: 49: 52.8$ & 1.213 & -8.090 & 14.074 & 0.571569 & 0.29 & 8.4 \\
\hline $\mathrm{J} 182107.83-335646.1 *$ & $18: 21: 07.83$ & $-33: 56: 46.1$ & -0.693 & -9.049 & 13.517 & 0.437311 & 0.36 & 5.6 \\
\hline J182109.83-364205.6* & $18: 21: 09.83$ & $-36: 42: 05.6$ & -3.191 & -10.284 & 14.289 & 0.552859 & 0.33 & 9.2 \\
\hline $\mathrm{J} 182111.41-314347.1 *$ & 18:21:11.41 & $-31: 43: 47.1$ & 1.313 & -8.059 & 14.636 & 0.564503 & 0.30 & 11.0 \\
\hline J182112.51-323530.4 & $18: 21: 12.51$ & $-32: 35: 30.4$ & 0.538 & -8.453 & 15.129 & 0.522630 & 0.22 & 13.4 \\
\hline J182115.76-332658.4 & $18: 21: 15.76$ & $-33: 26: 58.4$ & -0.231 & -8.850 & 14.677 & 0.619333 & 0.29 & 11.8 \\
\hline J182119.09-353204.7 & 18:21:19.09 & $-35: 32: 04.7$ & -2.115 & -9.794 & 13.307 & 0.746993 & 0.18 & 6.7 \\
\hline J182120.41-341205.1 & $18: 21: 20.41$ & $-34: 12: 05.1$ & -0.904 & -9.202 & 14.192 & 0.678528 & 0.21 & 9.8 \\
\hline J182122.11-362101.9 & $18: 21: 22.11$ & $-36: 21: 01.9$ & -2.853 & -10.166 & 13.839 & 0.729884 & 0.23 & 8.6 \\
\hline J182123.64-345030.5 & $8: 21: 23.64$ & $-34: 50: 30$ & -1.479 & -9.499 & 14.457 & 0.571765 & 0.2 & 10.2 \\
\hline J182126.33-342835.5 & $18: 21: 26.33$ & $-34: 28: 35.5$ & -1.144 & -9.344 & 14.107 & 0.554911 & 0.28 & 8.4 \\
\hline $\mathrm{J} 182128.21-345613.2$ & $18: 21: 28.21$ & $-34: 56: 13.2$ & -1.559 & -9.555 & 14.280 & 0.512818 & 0.30 & 8.8 \\
\hline J182129.07-343230.6 & $18: 21: 29.07$ & $-34: 32: 30.6$ & -1.199 & -9.381 & 13.922 & 0.541501 & 0.22 & 7.6 \\
\hline J182129.15-315014.7 & $18: 21: 29.15$ & $-31: 50: 14.7$ & 1.245 & -8.164 & 14.570 & 0.463979 & 0.40 & 9.6 \\
\hline $\mathrm{J} 182129.94-321151.5 *$ & $18: 21: 29.94$ & $-32: 11: 51.5$ & 0.921 & -8.329 & 13.805 & 0.556502 & 0.32 & 7.3 \\
\hline J182132.70-312954.0 & $18: 21: 32.70$ & $-31: 29: 54.0$ & 1.556 & -8.021 & 14.282 & 0.458739 & 0.33 & 8.3 \\
\hline J182138.46-330910.3 & $18: 21: 38.46$ & $-33: 09: 10.3$ & 0.073 & -8.787 & 14.273 & 0.456171 & 0.30 & 8.2 \\
\hline J182142.25-334005.2 & $18: 21: 42.25$ & $-33: 40: 05.2$ & -0.387 & -9.031 & 14.143 & 0.585484 & 0.31 & 8.8 \\
\hline $\mathrm{J} 182144.95-340940.9 *$ & $18: 21: 44.95$ & $-34: 09: 40.9$ & -0.829 & -9.260 & 13.922 & 0.474940 & 0.34 & 7.1 \\
\hline $\mathrm{J} 182150.94-313614.3 *$ & $18: 21: 50.94$ & $-31: 36: 14.3$ & 1.490 & -8.127 & 12.989 & 0.470252 & 0.32 & 4.5 \\
\hline J182151.52-340554.6* & $18: 21: 51.52$ & $-34: 05: 54.6$ & -0.762 & -9.252 & 14.432 & 0.443005 & 0.36 & 8.8 \\
\hline $\mathrm{J} 182153.77-342410.7 *$ & $18: 21: 53.77$ & $-34: 24: 10.7$ & -1.035 & -9.395 & 13.788 & 0.567302 & 0.25 & 7.3 \\
\hline
\end{tabular}


Table A.1. continued.

\begin{tabular}{|c|c|c|c|c|c|c|c|c|}
\hline VVV ID & $\begin{array}{l}\text { RA (J2000.0) } \\
\text { hh:mm:ss.ss }\end{array}$ & $\begin{array}{c}\text { Dec (J2000.0) } \\
\text { dd:mm:ss.s }\end{array}$ & $\begin{array}{c}\ell \\
(\mathrm{deg})\end{array}$ & $\begin{array}{c}b \\
(\mathrm{deg})\end{array}$ & $\begin{array}{l}\left\langle K_{\mathrm{s}}\right\rangle \\
(\mathrm{mag})\end{array}$ & $\begin{array}{c}P \\
\text { (days) }\end{array}$ & $\begin{array}{l}\text { Amplitude } \\
\text { (mag) }\end{array}$ & $\begin{array}{c}d \\
(\mathrm{kpc})\end{array}$ \\
\hline J182154.23-345156.0 & $18: 21: 54.23$ & $-34: 51: 56.0$ & -1.454 & -9.603 & 14.256 & 0.542029 & 0.24 & 9.0 \\
\hline $\mathrm{J} 182157.52-322432.2$ & $18: 21: 57.52$ & $-32: 24: 32.2$ & 0.775 & -8.512 & 14.413 & 0.570558 & 0.26 & 9.9 \\
\hline $\mathrm{J} 182200.35-322139.1 *$ & $18: 22: 00.35$ & $-32: 21: 39.1$ & 0.823 & -8.499 & 15.004 & 0.552370 & 0.35 & 13.0 \\
\hline J182201.27-334837.7 & $18: 22: 01.27$ & $-33: 48: 37.7$ & -0.486 & -9.153 & 15.140 & 0.569133 & 0.21 & 14.1 \\
\hline J182204.24-350828.6 & $18: 22: 04.24$ & $-35: 08: 28.6$ & -1.689 & -9.756 & 13.981 & 0.560444 & 0.25 & 8.0 \\
\hline $\mathrm{J} 182209.13-340440.9 *$ & $18: 22: 09.13$ & $-34: 04: 40.9$ & -0.716 & -9.297 & 14.331 & 0.540690 & 0.30 & 9.3 \\
\hline $\mathrm{J} 182209.79-321623.3 *$ & $18: 22: 09.79$ & $-32: 16: 23.3$ & 0.917 & -8.489 & 14.036 & 0.566502 & 0.32 & 8.3 \\
\hline $\mathrm{J} 182212.34-343822.8 *$ & $18: 22: 12.34$ & $-34: 38: 22.8$ & -1.220 & -9.558 & 13.982 & 0.583505 & 0.38 & 8.2 \\
\hline J182219.23-320547.9 & $18: 22: 19.23$ & $-32: 05: 47.9$ & 1.092 & -8.439 & 14.803 & 0.625904 & 0.20 & 12.6 \\
\hline J182219.90-321936.8* & $18: 22: 19.90$ & $-32: 19: 36.8$ & 0.885 & -8.545 & 14.250 & 0.437738 & 0.27 & 8.0 \\
\hline J182221.12-360109.1 & $18: 22: 21.12$ & $-36: 01: 09.1$ & -2.462 & -10.197 & 13.976 & 0.463379 & 0.32 & 7.2 \\
\hline J182222.95-311917.3 & $18: 22: 22.95$ & $-31: 19: 17.3$ & 1.797 & -8.100 & 14.468 & 0.816553 & 0.26 & 12.4 \\
\hline $\mathrm{J} 182225.05-310753.9 *$ & $18: 22: 25.05$ & $-31: 07: 53.9$ & 1.971 & -8.021 & 13.919 & 0.431326 & 0.38 & 6.7 \\
\hline J182225.80-353709.4 & $18: 22: 25.80$ & $-35: 37: 09.4$ & -2.090 & -10.034 & 14.354 & 0.597054 & 0.28 & 9.9 \\
\hline J182227.66-350457.5 & $18: 22: 27.66$ & $-35: 04: 57.5$ & -1.599 & -9.802 & 14.495 & 0.595086 & 0.20 & 10.6 \\
\hline J182229.70-315351.6 & $18: 22: 29.70$ & $-31: 53: 51.6$ & 1.288 & -8.382 & 14.587 & 0.496165 & 0.32 & 10.0 \\
\hline J182230.30-323249.7 & $18: 22: 30.30$ & $-32: 32: 49.7$ & 0.703 & -8.677 & 14.198 & 0.663993 & 0.23 & 9.7 \\
\hline $\mathrm{J} 182231.09-312237.5 *$ & $18: 22: 31.09$ & $-31: 22: 37.5$ & 1.760 & -8.151 & 14.159 & 0.560636 & 0.27 & 8.7 \\
\hline J182234.43-312937.8 & $18: 22: 34.43$ & $-31: 29: 37.8$ & 1.660 & -8.215 & 14.107 & 0.505738 & 0.30 & 8.0 \\
\hline $\mathrm{J} 182234.81-342039.2$ & $18: 22: 34.81$ & $-34: 20: 39.2$ & -0.917 & -9.495 & 14.045 & 0.591048 & 0.25 & 8.5 \\
\hline $\mathrm{J} 182238.52-333105.5 *$ & $18: 22: 38.52$ & $-33: 31: 05.5$ & -0.163 & -9.138 & 14.337 & 0.520172 & 0.24 & 9.1 \\
\hline $\mathrm{J} 182239.12-311226.6 *$ & $18: 22: 39.12$ & $-31: 12: 26.6$ & 1.926 & -8.100 & 13.973 & 0.463222 & 0.37 & 7.2 \\
\hline J182242.39-334600.7 & $18: 22: 42.39$ & $-33: 46: 00.7$ & -0.382 & -9.261 & 13.935 & 0.740662 & 0.22 & 9.1 \\
\hline $\mathrm{J} 182243.13-315352.7 *$ & $18: 22: 43.13$ & $-31: 53: 52.7$ & 1.310 & -8.425 & 14.278 & 0.529661 & 0.29 & 8.9 \\
\hline J182245.05-344516.3 & $18: 22: 45.05$ & $-34: 45: 16.3$ & -1.274 & -9.709 & 14.688 & 0.539200 & 0.23 & 11.0 \\
\hline $\mathrm{J} 182245.23-360331.8 *$ & $18: 22: 45.23$ & $-36: 03: 31.8$ & -2.461 & -10.287 & 14.111 & 0.484856 & 0.27 & 7.9 \\
\hline J182245.98-323042.2 & $18: 22: 45.98$ & $-32: 30: 42.2$ & 0.760 & -8.710 & 14.705 & 0.472932 & 0.24 & 10.3 \\
\hline J182248.34-350357.8 & $18: 22: 48.34$ & $-35: 03: 57.8$ & -1.552 & -9.858 & 13.100 & 0.520327 & 0.30 & 5.0 \\
\hline J182249.08-315002.6 & $18: 22: 49.08$ & $-31: 50: 02.6$ & 1.377 & -8.415 & 14.116 & 0.558329 & 0.29 & 8.5 \\
\hline J182250.01-322322.8 & $18: 22: 50.01$ & $-32: 23: 22.8$ & 0.877 & -8.668 & 13.426 & 0.740543 & 0.35 & 7.1 \\
\hline $\mathrm{J} 182251.81-313412.0 *$ & $18: 22: 51.81$ & $-31: 34: 12.0$ & 1.619 & -8.304 & 13.564 & 0.537086 & 0.26 & 6.4 \\
\hline J182257.57-335758.3 & $18: 22: 57.57$ & $-33: 57: 58.3$ & -0.539 & -9.397 & 14.830 & 0.658633 & 0.25 & 13.2 \\
\hline $\mathrm{J} 182302.20-335723.9 *$ & $18: 23: 02.20$ & $-33: 57: 23.9$ & -0.523 & -9.407 & 14.922 & 0.454223 & 0.30 & 11.2 \\
\hline $\mathrm{J} 182303.88-320110.0 *$ & $18: 23: 03.88$ & $-32: 01: 10.0$ & 1.233 & -8.545 & 14.902 & 0.492776 & 0.31 & 11.6 \\
\hline J182304.31-332916.4 & $18: 23: 04.31$ & $-33: 29: 16.4$ & -0.095 & -9.205 & 14.246 & 0.750604 & 0.26 & 10.6 \\
\hline $\mathrm{J} 182305.31-343303.0 *$ & $18: 23: 05.31$ & $-34: 33: 03.0$ & -1.058 & -9.681 & 14.444 & 0.669004 & 0.30 & 11.0 \\
\hline J182305.40-342713.2 & 18:23:05.40 & $-34: 27: 13.2$ & -0.969 & -9.638 & 13.988 & 0.561041 & 0.26 & 8.0 \\
\hline $\mathrm{J} 182308.58-341138.0 *$ & $18: 23: 08.58$ & $-34: 11: 38.0$ & -0.728 & -9.532 & 13.189 & 0.670084 & 0.26 & 6.0 \\
\hline J182309.73-325812.2 & $18: 23: 09.73$ & $-32: 58: 12.2$ & 0.383 & -8.990 & 14.331 & 0.475552 & 0.16 & 8.7 \\
\hline J182313.77-341617.6* & $18: 23: 13.77$ & $-34: 16: 17.6$ & -0.791 & -9.583 & 13.680 & 0.601341 & 0.35 & 7.2 \\
\hline J182315.13-334148.2 & $18: 23: 15.13$ & $-33: 41: 48.2$ & -0.267 & -9.331 & 15.229 & 0.594360 & 0.32 & 15.1 \\
\hline $\mathrm{J} 182315.19-314745.3 *$ & $18: 23: 15.19$ & $-31: 47: 45.3$ & 1.453 & -8.480 & 14.808 & 0.536406 & 0.27 & 11.6 \\
\hline $\mathrm{J} 182317.32-325428.2 *$ & $18: 23: 17.32$ & $-32: 54: 28.2$ & 0.451 & -8.986 & 14.729 & 0.520657 & 0.30 & 11.0 \\
\hline $\mathrm{J} 182317.92-311656.0 *$ & $18: 23: 17.92$ & $-31: 16: 56.0$ & 1.921 & -8.257 & 14.241 & 0.463960 & 0.30 & 8.2 \\
\hline J182318.14-305958.9 & $18: 23: 18.14$ & $-30: 59: 58.9$ & 2.176 & -8.131 & 14.124 & 0.606402 & 0.22 & 8.9 \\
\hline $\mathrm{J} 182320.10-333533.8 *$ & $18: 23: 20.10$ & $-33: 35: 33.8$ & -0.165 & -9.300 & 13.903 & 0.699433 & 0.36 & 8.7 \\
\hline J182324.38-331254.1 & $18: 23: 24.38$ & $-33: 12: 54.1$ & 0.184 & -9.145 & 14.382 & 0.527412 & 0.31 & 9.4 \\
\hline J182326.53-305406.0 & $18: 23: 26.53$ & $-30: 54: 06.0$ & 2.278 & -8.113 & 14.343 & 0.516919 & 0.27 & 9.1 \\
\hline J182331.06-354353.1 & $18: 23: 31.06$ & $-35: 43: 53.1$ & -2.093 & -10.282 & 14.172 & 0.730818 & 0.29 & 10.1 \\
\hline J182335.61-310104.8 & $18: 23: 35.61$ & $-31: 01: 04.8$ & 2.188 & -8.194 & 15.248 & 0.610728 & 0.30 & 15.5 \\
\hline $\mathrm{J} 182338.91-341148.1 *$ & $18: 23: 38.91$ & $-34: 11: 48.1$ & -0.684 & -9.627 & 13.433 & 0.645082 & 0.23 & 6.6 \\
\hline J182339.95-324655.5 & $18: 23: 39.95$ & $-32: 46: 55.5$ & 0.601 & -9.000 & 13.437 & 0.608010 & 0.26 & 6.4 \\
\hline $\mathrm{J} 182341.44-312549.7 *$ & $18: 23: 41.44$ & $-31: 25: 49.7$ & 1.826 & -8.399 & 14.318 & 0.522633 & 0.27 & 9.1 \\
\hline J182342.10-320423.4 & $18: 23: 42.10$ & $-32: 04: 23.4$ & 1.246 & -8.690 & 13.411 & 0.572702 & 0.30 & 6.1 \\
\hline $\mathrm{J} 182345.70-315420.3 *$ & $18: 23: 45.70$ & $-31: 54: 20.3$ & 1.403 & -8.626 & 14.079 & 0.447602 & 0.22 & 7.4 \\
\hline $\mathrm{J} 182345.83-315214.5$ & $18: 23: 45.83$ & $-31: 52: 14.5$ & 1.435 & -8.611 & 14.015 & 0.535097 & 0.33 & 7.9 \\
\hline $\mathrm{J} 182350.13-335639.4 *$ & $18: 23: 50.13$ & $-33: 56: 39.4$ & -0.437 & -9.550 & 14.241 & 0.473344 & 0.25 & 8.3 \\
\hline J182350.17-305256.7 & $18: 23: 50.17$ & $-30: 52: 56.7$ & 2.334 & -8.180 & 14.416 & 0.522974 & 0.30 & 9.5 \\
\hline J182355.87-323731.7 & $18: 23: 55.87$ & $-32: 37: 31.7$ & 0.768 & -8.980 & 13.084 & 0.625512 & 0.24 & 5.5 \\
\hline J182356.27-320723.9* & $18: 23: 56.27$ & $-32: 07: 23.9$ & 1.223 & -8.757 & 13.732 & 0.470526 & 0.35 & 6.4 \\
\hline
\end{tabular}


F. Gran et al.: Mapping the outer bulge with RRab stars from the VVV Survey

Table A.1. continued

\begin{tabular}{|c|c|c|c|c|c|c|c|c|}
\hline VVV ID & $\begin{array}{l}\text { RA (J2000.0) } \\
\text { hh:mm:ss.ss }\end{array}$ & $\begin{array}{c}\text { Dec (J2000.0) } \\
\text { dd:mm:ss.s }\end{array}$ & $\begin{array}{c}\ell \\
(\operatorname{deg})\end{array}$ & $\begin{array}{c}b \\
(\mathrm{deg})\end{array}$ & $\begin{array}{l}\left\langle K_{\mathrm{s}}\right\rangle \\
(\mathrm{mag})\end{array}$ & $\begin{array}{c}P \\
\text { (days) }\end{array}$ & $\begin{array}{l}\text { Amplitude } \\
\text { (mag) }\end{array}$ & $\begin{array}{c}d \\
(\mathrm{kpc})\end{array}$ \\
\hline $\mathrm{J} 182400.52-315930.3$ & $18: 24: 00.52$ & $-31: 59: 30.3$ & 1.349 & -8.711 & 15.387 & 0.530904 & 0.33 & 15.3 \\
\hline $\mathrm{J} 182402.88-330313.4$ & $18: 24: 02.88$ & $-33: 03: 13.4$ & 0.391 & -9.193 & 14.490 & 0.603573 & 0.30 & 10.6 \\
\hline J182405.17-342148.9 & $18: 24: 05.17$ & $-34: 21: 48.9$ & -0.795 & -9.782 & 13.999 & 0.537014 & 0.26 & 7.9 \\
\hline J182405.27-313547.9* & $18: 24: 05.27$ & $-31: 35: 47.9$ & 1.714 & -8.549 & 14.168 & 0.515063 & 0.31 & 8.4 \\
\hline $\mathrm{J} 182410.61-325402.0$ & $18: 24: 10.61$ & $-32: 54: 02.0$ & 0.542 & -9.149 & 14.440 & 0.573694 & 0.26 & 10.1 \\
\hline $\mathrm{J} 182411.73-302708.1$ & $18: 24: 11.73$ & $-30: 27: 08.1$ & 2.757 & -8.055 & 14.620 & 0.473788 & 0.36 & 9.9 \\
\hline J182416.45-325426.8 & $18: 24: 16.45$ & $-32: 54: 26.8$ & 0.545 & -9.171 & 13.982 & 0.483269 & 0.30 & 7.4 \\
\hline J182417.26-333034.6 & $18: 24: 17.26$ & $-33: 30: 34.6$ & -0.000 & -9.441 & 13.783 & 0.563393 & 0.31 & 7.3 \\
\hline $\mathrm{J} 182418.43-343055.4 *$ & $18: 24: 18.43$ & $-34: 30: 55.4$ & -0.913 & -9.890 & 14.527 & 0.564528 & 0.28 & 10.4 \\
\hline J182421.20-314537.3 & $18: 24: 21.20$ & $-31: 45: 37.3$ & 1.592 & -8.673 & 15.230 & 0.493408 & 0.33 & 13.7 \\
\hline $\mathrm{J} 182423.75-323912.0 *$ & $18: 24: 23.75$ & $-32: 39: 12.0$ & 0.787 & -9.080 & 14.068 & 0.568544 & 0.21 & 8.4 \\
\hline $\mathrm{J} 182424.45-341657.6 *$ & $18: 24: 24.45$ & $-34: 16: 57.6$ & -0.692 & -9.806 & 14.303 & 0.524002 & 0.28 & 9.0 \\
\hline $\mathrm{J} 182427.70-315252.5 *$ & $18: 24: 27.70$ & $-31: 52: 52.5$ & 1.493 & -8.748 & 14.588 & 0.557001 & 0.32 & 10.7 \\
\hline J182429.56-304152.8 & $18: 24: 29.56$ & $-30: 41: 52.8$ & 2.565 & -8.223 & 14.539 & 0.521436 & 0.34 & 10.1 \\
\hline J182434.52-312942.4 & $18: 24: 34.52$ & $-31: 29: 42.4$ & 1.853 & -8.596 & & 0.480206 & 0.30 & 7.0 \\
\hline J182437.59-300923.1 & $18: 24: 37.59$ & $-30: 09: 23.1$ & 3.066 & -8.004 & 14.957 & 0.531333 & 0.37 & 12.5 \\
\hline J182440.84-342827.2 & $18: 24: 40.84$ & $-34: 28: 27.2$ & -0.841 & -9.941 & 14.342 & 0.550343 & 0.29 & 9.4 \\
\hline $\mathrm{J} 182441.83-324524.7 *$ & $18: 24: 41.83$ & $-32: 45: 24.7$ & 0.722 & -9.183 & 14.176 & 0.527961 & 0.33 & 8.5 \\
\hline $\mathrm{J} 182445.92-320258.6 *$ & $18: 24: 45.92$ & $-32: 02: 58.6$ & 1.369 & -8.881 & 14.338 & 0.511776 & 0.30 & 9.0 \\
\hline J182447.41-325009.0 & $18: 24: 47.41$ & $-32: 50: 09.0$ & 0.659 & -9.236 & 13.987 & 0.535326 & 0.34 & 7.8 \\
\hline $\mathrm{J} 182449.18-322704.7$ & $18: 24: 49.18$ & $-32: 27: 04.7$ & 1.011 & -9.070 & 14.130 & 0.544808 & 0.26 & 8.5 \\
\hline J182451.06-340703.4 & $18: 24: 51.06$ & $-34: 07: 03$ & -0.5 & -9.8 & 15.6 & 0.6233 & & 18.8 \\
\hline J182455.32-331043.6 & $18: 24: 55.32$ & $-33: 10: 43.6$ & 0.360 & -9.413 & 14.166 & 0.587058 & 0.26 & 9.0 \\
\hline $\mathrm{J} 182456.37-344158.5 *$ & $18: 24: 56.37$ & $-34: 41: 58.5$ & -1.022 & -10.088 & 13.732 & 0.535269 & 0.32 & 6.9 \\
\hline J182501.64-323712.8 & $: 01.64$ & -32 : & 0.877 & -9.185 & 14.0 & 0.60 & 0.18 & 8.8 \\
\hline $502.15-332926.7$ & 02.15 & $-33: 29: 26.7$ & 0.0 & -9.5 & 14.809 & 0.522656 & 0.25 & 11.5 \\
\hline $507.13-323025.5$ & $18: 25: 07.13$ & $-32: 30: 2$ & 0.989 & -9.151 & 14.096 & 0.507882 & 0.34 & 8.0 \\
\hline J182507.66-331200.3 & $18: 25: 07.66$ & $-33: 12: 00.3$ & & -9.461 & 14.409 & 0.652371 & 0.27 & 10.7 \\
\hline $\mathrm{J} 182508.46-310451.3 *$ & $18: 25: 08.46$ & $-31: 04: 51.3$ & 2.282 & -8.519 & 14.271 & 0.528510 & 0.31 & 8.9 \\
\hline J182509.55-322852.0 & $18: 25: 09.55$ & $-32: 28: 52.0$ & 1.016 & -9.148 & 13.758 & 0.702716 & 0.27 & 8.1 \\
\hline $\mathrm{J} 182510.15-332547.6 *$ & $18: 25: 10.15$ & $-33: 25: 47.6$ & 0.155 & -9.571 & 14.308 & 0.519095 & 0.30 & 9.0 \\
\hline J182512.86-313713.6 & $18: 25: 12.86$ & $-31: 37: 13.6$ & 1.801 & -8.774 & 14.880 & 0.493223 & 0.34 & 11.5 \\
\hline $\mathrm{J} 182515.85-314307.1$ & $18: 25: 15.85$ & $-31: 43: 07.1$ & 1.717 & -8.828 & 14.360 & 0.624496 & 0.33 & 10.2 \\
\hline $\mathrm{J} 182518.77-342627.4$ & $18: 25: 18.77$ & $-34: 26: 27.4$ & -0.752 & -10.044 & 15.437 & 0.580120 & 0.24 & 16.5 \\
\hline $\mathrm{J} 182522.33-322803.5$ & $18: 25: 22.33$ & $-32: 28: 03.5$ & & -9.182 & 13.245 & 0.498788 & 0.24 & 5.3 \\
\hline $\mathrm{J} 182522.56-333047.8 *$ & $18: 25: 22.56$ & $-33: 30: 47.8$ & & -9.646 & 14.073 & 0.539255 & 0.34 & 8.2 \\
\hline $\mathrm{J} 182523.31-314242.1$ & $18: 25: 23.31$ & $-31: 42: 42.1$ & 1.735 & -8.848 & 15.472 & 0.668901 & 0.30 & 18.1 \\
\hline $\mathrm{J} 182524.55-305632.3 *$ & $18: 25: 24.55$ & $-30: 56: 32.3$ & 2.433 & -8.508 & 14.353 & 0.643124 & 0.33 & 10.3 \\
\hline $\mathrm{J} 182527.87-333306.2 *$ & $18: 25: 27.87$ & $-33: 33: 06.2$ & 0.072 & -9.680 & 14.019 & 0.678785 & 0.25 & 9.0 \\
\hline $\mathrm{J} 182531.20-305030.6 *$ & $18: 25: 31.20$ & $-30: 50: 30.6$ & 2.535 & -8.484 & 14.411 & 0.554115 & 0.25 & 9.8 \\
\hline $\mathrm{J} 182532.01-300107.2 *$ & $18: 25: 32.01$ & $-30: 01: 07.2$ & 3.280 & -8.118 & 13.283 & 0.551809 & 0.32 & 5.7 \\
\hline $\mathrm{J} 182532.83-305056.7 *$ & $18: 25: 32.83$ & $-30: 50: 56.7$ & 2.531 & -8.493 & 14.302 & 0.564149 & 0.22 & 9.4 \\
\hline J182534.29-313918.6 & $18: 25: 34.29$ & $-31: 39: 18.6$ & 1.804 & -8.858 & 14.723 & 0.482623 & 0.28 & 10.6 \\
\hline J182537.23-301959.3 & $18: 25: 37.23$ & $-30: 19: 59.3$ & 3.004 & -8.276 & 13.491 & 0.588256 & 0.28 & 6.5 \\
\hline J182538.37-295919.2 & $18: 25: 38.37$ & $-29: 59: 19.2$ & 3.317 & -8.125 & 14.146 & 0.536907 & 0.30 & 8.5 \\
\hline $\mathrm{J} 182538.73-310836.5 *$ & $18: 25: 38.73$ & $-31: 08: 36.5$ & 2.274 & -8.643 & 14.359 & 0.692789 & 0.30 & 10.8 \\
\hline $\mathrm{J} 182541.00-314838.5 *$ & $18: 25: 41.00$ & $-31: 48: 38.5$ & 1.674 & -8.948 & 13.429 & 0.542820 & 0.30 & 6.0 \\
\hline J182543.27-341255.4 & $18: 25: 43.27$ & $-34: 12: 55.4$ & -0.509 & -10.020 & 13.826 & 0.602439 & 0.24 & 7.7 \\
\hline $\mathrm{J} 182543.95-340010.3$ & $18: 25: 43.95$ & $-34: 00: 10.3$ & -0.3 & -9.929 & 14.1 & 0.514020 & 0.33 & 8.3 \\
\hline $\mathrm{J} 182544.94-330651.3$ & $18: 25: 44.94$ & $-33: 06: 51.3$ & 0.497 & -9.540 & 14.359 & 0.502833 & 0.27 & 9.0 \\
\hline $\mathrm{J} 182546.72-331638.3$ & $18: 25: 46.72$ & $-33: 16: 38.3$ & 0.351 & -9.617 & 13.846 & 0.509204 & 0.33 & 7.1 \\
\hline J182547.88-294930.4 & $18: 25: 47.88$ & $-29: 49: 30.4$ & 3.480 & -8.082 & 14.273 & 0.528164 & 0.30 & 8.9 \\
\hline $\mathrm{J} 182548.30-313947.8 *$ & $18: 25: 48.30$ & $-31: 39: 47.8$ & 1.819 & -8.906 & 13.545 & 0.571442 & 0.36 & 6.5 \\
\hline J182549.95-311804.1 & $18: 25: 49.95$ & $-31: 18: 04.1$ & 2.150 & -8.750 & 13.878 & 0.563409 & 0.27 & 7.6 \\
\hline J182556.09-321250.0 & $18: 25: 56.09$ & $-32: 12: 50.0$ & 1.332 & -9.175 & 14.883 & 0.537141 & 0.23 & 12.1 \\
\hline $\mathrm{J} 182556.37-320926.8 *$ & $18: 25: 56.37$ & $-32: 09: 26.8$ & 1.384 & -9.151 & 13.727 & 0.640578 & 0.32 & 7.6 \\
\hline J182558.42-303555.0 & $18: 25: 58.42$ & $-30: 35: 55.0$ & 2.799 & -8.463 & 14.939 & 0.475527 & 0.28 & 11.6 \\
\hline $\mathrm{J} 182558.66-333527.9 *$ & $18: 25: 58.66$ & $-33: 35: 27.9$ & 0.084 & -9.793 & 14.256 & 0.537678 & 0.29 & 8.9 \\
\hline $\mathrm{J} 182605.38-321939.7 *$ & $18: 26: 05.38$ & $-32: 19: 39.7$ & 1.244 & -9.255 & 14.798 & 0.587475 & 0.31 & 12.2 \\
\hline J182606.29-305412.3 & $18: 26: 06.29$ & $-30: 54: 12.3$ & 2.536 & -8.624 & 14.284 & 0.513302 & 0.27 & 8.8 \\
\hline
\end{tabular}


Table A.1. continued.

\begin{tabular}{|c|c|c|c|c|c|c|c|c|}
\hline VVV ID & $\begin{array}{l}\text { RA (J2000.0) } \\
\text { hh:mm:ss.ss }\end{array}$ & $\begin{array}{c}\text { Dec (J2000.0) } \\
\text { dd:mm:ss.s }\end{array}$ & $\begin{array}{c}\ell \\
(\mathrm{deg})\end{array}$ & $\begin{array}{c}b \\
(\mathrm{deg})\end{array}$ & $\begin{array}{l}\left\langle K_{\mathrm{s}}\right\rangle \\
(\mathrm{mag})\end{array}$ & $\begin{array}{c}P \\
\text { (days) }\end{array}$ & $\begin{array}{l}\text { Amplitude } \\
\text { (mag) }\end{array}$ & $\begin{array}{c}d \\
(\mathrm{kpc})\end{array}$ \\
\hline J182607.09-321309.4 & 18:26:07.09 & $-32: 13: 09.4$ & 1.345 & -9.213 & 14.328 & 0.490855 & 0.30 & 8.8 \\
\hline $\mathrm{J} 182607.78-312211.2 *$ & $18: 26: 07.78$ & $-31: 22: 11.2$ & 2.116 & -8.837 & 13.731 & 0.595912 & 0.27 & 7.3 \\
\hline J182608.84-303346.1 & $18: 26: 08.84$ & $-30: 33: 46.1$ & 2.848 & -8.480 & 13.765 & 0.493715 & 0.24 & 6.7 \\
\hline $\mathrm{J} 182609.06-334753.2$ & $18: 26: 09.06$ & $-33: 47: 53.2$ & -0.088 & -9.916 & 14.122 & 0.697725 & 0.31 & 9.6 \\
\hline $\mathrm{J} 182609.53-321657.6$ & $18: 26: 09.53$ & $-32: 16: 57.6$ & 1.291 & -9.248 & 14.581 & 0.534625 & 0.35 & 10.4 \\
\hline J182609.71-302815.0 & $18: 26: 09.71$ & $-30: 28: 15.0$ & 2.933 & -8.442 & 13.827 & 0.643770 & 0.37 & 8.0 \\
\hline J182613.54-303101.2 & $18: 26: 13.54$ & $-30: 31: 01.2$ & 2.897 & -8.475 & 14.107 & 0.639313 & 0.28 & 9.1 \\
\hline J182615.34-311358.0 & $18: 26: 15.34$ & $-31: 13: 58.0$ & 2.252 & -8.800 & 14.382 & 0.485307 & 0.34 & 9.0 \\
\hline $\mathrm{J} 182622.04-342942.5 *$ & $18: 26: 22.04$ & $-34: 29: 42.5$ & -0.705 & -10.263 & 14.005 & 0.477439 & 0.34 & 7.4 \\
\hline J182627.39-322459.8 & $18: 26: 27.39$ & $-32: 24: 59.8$ & 1.198 & -9.364 & 14.754 & 0.624672 & 0.30 & 12.3 \\
\hline J182627.79-304558.7 & $18: 26: 27.79$ & $-30: 45: 58.7$ & 2.695 & -8.632 & 13.930 & 0.443626 & 0.38 & 6.9 \\
\hline $\mathrm{J} 182627.86-332124.7 *$ & $18: 26: 27.86$ & $-33: 21: 24.7$ & 0.343 & -9.781 & 14.611 & 0.409182 & 0.30 & 9.1 \\
\hline J182629.13-335625.3 & $18: 26: 29.13$ & $-33: 56: 25.3$ & -0.187 & -10.041 & 14.184 & 0.559946 & 0.26 & 8.8 \\
\hline $\mathrm{J} 182631.34-300210.2$ & $18: 26: 31.34$ & $-30: 02: 10.2$ & 3.361 & -8.317 & 14.744 & 0.531141 & 0.23 & 11.2 \\
\hline J182631.38-325030.6 & $18: 26: 31.38$ & $-32: 50: 30.6$ & 0.817 & -9.565 & 13.993 & 0.611636 & 0.18 & 8.4 \\
\hline $\mathrm{J} 182632.31-314222.7 *$ & $18: 26: 32.31$ & $-31: 42: 22.7$ & 1.850 & -9.065 & 14.233 & 0.533296 & 0.26 & 8.8 \\
\hline J182636.17-322629.1 & $18: 26: 36.17$ & $-32: 26: 29.1$ & 1.189 & -9.403 & 14.434 & 0.594569 & 0.21 & 10.3 \\
\hline $\mathrm{J} 182645.41-314838.8 *$ & $18: 26: 45.41$ & $-31: 48: 38.8$ & 1.776 & -9.153 & 14.247 & 0.483908 & 0.33 & 8.4 \\
\hline $\mathrm{J} 182650.14-310954.7 *$ & $18: 26: 50.14$ & $-31: 09: 54.7$ & 2.370 & -8.881 & 13.974 & 0.580077 & 0.29 & 8.1 \\
\hline $\mathrm{J} 182655.52-325844.0$ & $18: 26: 55.52$ & $-32: 58: 44.0$ & 0.730 & -9.701 & 12.981 & 0.416512 & 0.30 & 4.2 \\
\hline J182655.74-295956.1 & $18: 26: 55.74$ & $-29: 59: 56.1$ & 3.434 & -8.379 & 14.149 & 0.616101 & 0.34 & 9.1 \\
\hline J182700.00-340417.5 & $18: 27: 00.00$ & $-34: 04: 17.5$ & -0.259 & -10.195 & 15.319 & 0.589598 & 0.30 & 15.7 \\
\hline J182706.06-295149.4 & $18: 27: 06.06$ & $-29: 51: 49.4$ & 3.574 & -8.352 & 14.702 & 0.488993 & 0.21 & 10.5 \\
\hline J182711.26-293150.5 & $18: 27: 11.26$ & $-29: 31: 50.5$ & 3.883 & -8.219 & 14.604 & 0.534614 & 0.31 & 10.5 \\
\hline J182715.28-295654.2 & $18: 27: 15.28$ & $-29: 56: 54.2$ & 3.512 & -8.419 & 14.824 & 0.653992 & 0.25 & 13.1 \\
\hline J182717.98-331954.4 & $18: 27: 17.98$ & $-33: 19: 54.4$ & 0.444 & -9.927 & 14.355 & 0.513735 & 0.32 & 9.1 \\
\hline $\mathrm{J} 182718.43-285937.0 *$ & $18: 27: 18.43$ & $-28: 59: 37.0$ & 4.380 & -8.002 & 13.918 & 0.693611 & 0.33 & 8.7 \\
\hline J182719.92-293305.1 & $18: 27: 19.92$ & $-29: 33: 05.1$ & 3.878 & -8.257 & 12.782 & 0.624945 & 0.33 & 4.7 \\
\hline $\mathrm{J} 182723.88-321408.8 *$ & $18: 27: 23.88$ & $-32: 14: 08.8$ & 1.451 & -9.463 & 14.087 & 0.535149 & 0.19 & 8.2 \\
\hline J182724.85-311030.6 & $18: 27: 24.85$ & $-31: 10: 30.6$ & 2.416 & -8.996 & 14.142 & 0.498030 & 0.33 & 8.1 \\
\hline J182726.29-332519.9 & $18: 27: 26.29$ & $-33: 25: 19.9$ & 0.374 & -9.992 & 13.836 & 0.626060 & 0.27 & 7.9 \\
\hline $\mathrm{J} 182728.38-334444.1 *$ & $18: 27: 28.38$ & $-33: 44: 44.1$ & 0.082 & -10.14 & 15.173 & 0.493632 & 0.36 & 13.3 \\
\hline J182729.91-335147.1 & $18: 27: 29.91$ & $-33: 51: 47.1$ & -0.023 & -10.196 & 13.743 & 0.664991 & 0.25 & 7.8 \\
\hline J182730.35-320024.7 & $18: 27: 30.35$ & $-32: 00: 24.7$ & 1.670 & -9.382 & 14.889 & 0.643091 & 0.24 & 13.4 \\
\hline J182731.27-315856.3 & $18: 27: 31.27$ & $-31: 58: 56.3$ & 1.693 & -9.374 & 15.542 & 0.639337 & 0.22 & 18.3 \\
\hline $\mathrm{J} 182733.11-322845.3 *$ & $18: 27: 33.11$ & $-32: 28: 45.3$ & 1.244 & -9.599 & 14.077 & 0.558541 & 0.30 & 8.4 \\
\hline $\mathrm{J} 182733.14-331057.0 *$ & $18: 27: 33.14$ & $-33: 10: 57.0$ & 0.603 & -9.908 & 14.177 & 0.487500 & 0.32 & 8.1 \\
\hline J182735.49-293132.2 & $18: 27: 35.49$ & $-29: 31: 32.2$ & 3.927 & -8.296 & 14.007 & 0.584505 & 0.30 & 8.3 \\
\hline J182737.93-333604.6 & $18: 27: 37.93$ & $-33: 36: 04.6$ & 0.228 & -10.107 & 13.448 & 0.607024 & 0.20 & 6.4 \\
\hline $\mathrm{J} 182742.02-333032.0$ & $18: 27: 42.02$ & $-33: 30: 32.0$ & 0.319 & -10.079 & 13.757 & 0.492363 & 0.25 & 6.7 \\
\hline J182743.30-290221.1 & $18: 27: 43.30$ & $-29: 02: 21.1$ & 4.380 & -8.104 & 14.239 & 0.537845 & 0.29 & 8.9 \\
\hline J182745.01-312201.4 & $18: 27: 45.01$ & $-31: 22: 01.4$ & 2.274 & -9.146 & 14.277 & 0.446076 & 0.28 & 8.2 \\
\hline $\mathrm{J} 182748.74-333149.7 *$ & $18: 27: 48.74$ & $-33: 31: 49.7$ & 0.310 & -10.110 & 14.147 & 0.524216 & 0.38 & 8.4 \\
\hline J182748.94-332826.0 & $18: 27: 48.94$ & $-33: 28: 26.0$ & 0.362 & -10.086 & 14.217 & 0.540570 & 0.34 & 8.8 \\
\hline J182750.07-291747.4 & $18: 27: 50.07$ & $-29: 17: 47.4$ & 4.158 & -8.241 & 14.255 & 0.475663 & 0.32 & 8.4 \\
\hline J182754.52-304513.0 & $18: 27: 54.52$ & $-30: 45: 13.0$ & 2.846 & -8.904 & 14.233 & 0.508730 & 0.20 & 8.6 \\
\hline J182800.86-294426.6 & $18: 28: 00.86$ & $-29: 44: 26.6$ & 3.774 & -8.474 & 14.601 & 0.594099 & 0.25 & 11.1 \\
\hline $\mathrm{J} 182801.66-331118.4 *$ & 18:28:01.66 & $-33: 11: 18.4$ & 0.642 & -10.000 & 14.042 & 0.554896 & 0.31 & 8.2 \\
\hline J182802.20-323429.1 & $18: 28: 02.20$ & $-32: 34: 29.1$ & 1.203 & -9.733 & 13.588 & 0.734446 & 0.26 & 7.6 \\
\hline J182805.83-303916.6 & $18: 28: 05.83$ & $-30: 39: 16.6$ & 2.954 & -8.897 & 13.904 & 0.518065 & 0.30 & 7.4 \\
\hline J182805.92-290328.0 & 18:28:05.92 & $-29: 03: 28.0$ & 4.400 & -8.186 & 14.844 & 0.439228 & 0.34 & 10.6 \\
\hline J182808.70-320319.5 & $18: 28: 08.70$ & $-32: 03: 19.5$ & 1.686 & -9.525 & 14.796 & 0.639672 & 0.30 & 12.7 \\
\hline J182814.13-320518.7 & $18: 28: 14.13$ & $-32: 05: 18.7$ & 1.664 & -9.557 & 14.244 & 0.683667 & 0.33 & 10.1 \\
\hline J182814.98-332949.7 & $18: 28: 14.98$ & $-33: 29: 49.7$ & 0.381 & -10.177 & 13.459 & 0.590455 & 0.22 & 6.4 \\
\hline J182817.73-293419.3 & $18: 28: 17.73$ & $-29: 34: 19.3$ & 3.954 & -8.453 & 14.694 & 0.569684 & 0.22 & 11.4 \\
\hline J182818.27-295349.9 & $18: 28: 18.27$ & $-29: 53: 49.9$ & 3.661 & -8.600 & 14.796 & 0.550982 & 0.28 & 11.7 \\
\hline J182818.95-294210.4 & $18: 28: 18.95$ & $-29: 42: 10.4$ & 3.838 & -8.516 & 13.879 & 0.677746 & 0.30 & 8.4 \\
\hline J182824.55-291328.3* & $18: 28: 24.55$ & $-29: 13: 28.3$ & 4.280 & -8.321 & 13.091 & 0.559538 & 0.28 & 5.2 \\
\hline J182825.18-304859.7 & $18: 28: 25.18$ & $-30: 48: 59.7$ & 2.838 & -9.030 & 15.109 & 0.459897 & 0.28 & 12.4 \\
\hline J182826.80-304618.4 & $18: 28: 26.80$ & $-30: 46: 18.4$ & 2.882 & -9.016 & 14.520 & 0.479108 & 0.38 & 9.5 \\
\hline
\end{tabular}


F. Gran et al.: Mapping the outer bulge with RRab stars from the VVV Survey

Table A.1. continued.

\begin{tabular}{|c|c|c|c|c|c|c|c|c|}
\hline VVV ID & $\begin{array}{c}\text { RA (J2000.0) } \\
\text { hh:mm:ss.ss }\end{array}$ & $\begin{array}{c}\text { Dec (J2000.0) } \\
\text { dd:mm:ss.s }\end{array}$ & $\begin{array}{c}\ell \\
(\mathrm{deg})\end{array}$ & $\begin{array}{c}b \\
(\mathrm{deg})\end{array}$ & $\begin{array}{c}\left\langle K_{\mathrm{s}}\right\rangle \\
(\mathrm{mag})\end{array}$ & $\begin{array}{c}P \\
\text { (days) }\end{array}$ & $\begin{array}{l}\text { Amplitude } \\
\text { (mag) }\end{array}$ & $\begin{array}{c}d \\
(\mathrm{kpc})\end{array}$ \\
\hline $\mathrm{J} 182828.73-330519.7 *$ & $18: 28: 28.73$ & $-33: 05: 19.7$ & 0.775 & -10.042 & 13.838 & 0.580134 & 0.32 & 7.6 \\
\hline J182829.11-295836.2 & $18: 28: 29.11$ & $-29: 58: 36.2$ & 3.606 & -8.670 & 14.601 & 0.544609 & 0.32 & 10.6 \\
\hline J182829.60-285430.9 & $18: 28: 29.60$ & $-28: 54: 30.9$ & 4.574 & -8.196 & 14.077 & 0.475703 & 0.24 & 7.7 \\
\hline J182829.65-303455.0 & $18: 28: 29.65$ & $-30: 34: 55.0$ & 3.059 & -8.941 & 14.385 & 0.635021 & 0.26 & 10.4 \\
\hline J182831.16-283358.6 & $18: 28: 31.16$ & $-28: 33: 58.6$ & 4.886 & -8.048 & 14.523 & 0.501339 & 0.35 & 9.8 \\
\hline $\mathrm{J} 182831.99-311120.0 *$ & $18: 28: 31.99$ & $-31: 11: 20.0$ & 2.511 & -9.217 & 15.116 & 0.506837 & 0.26 & 13.1 \\
\hline J182833.53-284322.2 & $18: 28: 33.53$ & $-28: 43: 22.2$ & 4.748 & -8.126 & 15.018 & 0.759659 & 0.21 & 15.6 \\
\hline $\mathrm{J} 182839.23-322348.4 *$ & $18: 28: 39.23$ & $-32: 23: 48.4$ & 1.423 & -9.772 & 14.087 & 0.509315 & 0.26 & 8.0 \\
\hline $\mathrm{J} 182842.86-324040.5$ & $18: 28: 42.86$ & $-32: 40: 40.5$ & 1.172 & -9.906 & 14.745 & 0.583376 & 0.28 & 11.8 \\
\hline J182842.87-295751.5 & $18: 28: 42.87$ & $-29: 57: 51.5$ & 3.640 & -8.709 & 14.990 & 0.445537 & 0.27 & 11.5 \\
\hline J182846.73-312239.8 & $18: 28: 46.73$ & $-31: 22: 39.8$ & 2.363 & -9.347 & 14.176 & 0.589018 & 0.24 & 9.0 \\
\hline J182847.97-290533.2 & $18: 28: 47.97$ & $-29: 05: 33.2$ & 4.438 & -8.338 & 14.001 & 0.569376 & 0.30 & 8.1 \\
\hline J182848.51-285237.2 & $18: 28: 48.51$ & $-28: 52: 37.2$ & 4.634 & -8.244 & 13.600 & 0.503692 & 0.35 & 6.3 \\
\hline J182849.89-291848.5 & $18: 28: 49.89$ & $-29: 18: 48.5$ & 4.241 & -8.443 & 13.637 & 0.655261 & 0.36 & 7.4 \\
\hline J182852.02-295715.5 & $18: 28: 52.02$ & $-29: 57: 15.5$ & 3.664 & -8.735 & 14.753 & 0.390274 & 0.40 & 9.5 \\
\hline J182852.12-282200.5 & $18: 28: 52.12$ & $-28: 22: 00.5$ & 5.101 & -8.028 & 14.173 & 0.481837 & 0.36 & 8.1 \\
\hline $\mathrm{J} 182852.35-323916.3 *$ & $18: 28: 52.35$ & $-32: 39: 16.3$ & 1.208 & -9.926 & 14.247 & 0.527629 & 0.27 & 8.8 \\
\hline $\mathrm{J} 182853.34-312151.2 *$ & $18: 28: 53.34$ & $-31: 21: 51.2$ & 2.386 & -9.362 & 14.449 & 0.449386 & 0.34 & 8.9 \\
\hline $\mathrm{J} 182853.82-325251.9 *$ & $18: 28: 53.82$ & $-32: 52: 51.9$ & 1.004 & -10.030 & 14.172 & 0.567524 & 0.30 & 8.8 \\
\hline J182903.73-285810.0 & $18: 29: 03.73$ & $-28: 58: 10.0$ & 4.575 & -8.335 & 14.618 & 0.445788 & 0.36 & 9.6 \\
\hline J182905.19-331315.6 & 18:29:05.19 & $-33: 13: 15.6$ & 0.711 & -10.214 & 13.990 & 0.628587 & 0.2 & 8.5 \\
\hline $\mathrm{J} 182905.21-312636.2 *$ & 18:29:05.21 & $-31: 26: 36.2$ & 2.332 & -9.435 & 14.426 & 0.5478 & & 9.8 \\
\hline J182905.60-303255.3 & 18:29:05.60 & $-30: 32: 55.3$ & 3.147 & -9.042 & 13.964 & 0.577297 & & 8.1 \\
\hline J182912.14-311354.2 & $18: 29: 12.14$ & $-31: 13: 54.2$ & 2.536 & -9.364 & 15.011 & 0.591307 & 0.24 & 13.5 \\
\hline J182922.41-30 & 22.41 & $-30: 19: 39.4$ & 3.374 & -8.998 & 14.179 & 0.4 & 0.35 & 8.0 \\
\hline J182923.84-303933.9 & $18: 29: 23.84$ & $-30: 39: 33.9$ & 3.075 & -9.149 & 14.175 & 0.482 & 0.31 & 8.1 \\
\hline J182926.73-284124.0 & $18: 29: 26.73$ & $-28: 41: 24.0$ & 4.866 & -8.285 & 14.417 & 0.485 & 0.29 & 9.1 \\
\hline J182930.05-284122.8 & $18: 29: 30.05$ & $-28: 41: 22.8$ & 4.871 & -8.2 & 13. & 0.612486 & 0.23 & 7.5 \\
\hline J182931.66-322656.1 & $18: 29: 31.66$ & $-32: 26: 56.1$ & 1.457 & -9.960 & 13.985 & 0.511444 & 0.31 & 7.6 \\
\hline $\mathrm{J} 182932.16-300956.8 *$ & $18: 29: 32.16$ & $-30: 09: 56.8$ & 3.537 & -8.958 & 13.572 & 0.452104 & & 5.8 \\
\hline J182935.49-291532.8 & $18: 29: 35.49$ & $-29: 15: 32.8$ & 4.365 & -8.567 & 14.036 & 0.529532 & 0.24 & 8.0 \\
\hline J182937.37-320407.5 & $18: 29: 37.37$ & $-32: 04: 07.5$ & 1.813 & -9.812 & 14.175 & 0.514485 & 0.21 & 8.4 \\
\hline J182939.23-313815.8 & $18: 29: 39.23$ & $-31: 38: 15.8$ & 2.209 & -9.629 & 14.765 & 0.564487 & 0.36 & 11.7 \\
\hline J182943.45-281544.2 & $18: 29: 43.45$ & $-28: 15: 44.2$ & 5.280 & -8.149 & 14.625 & 0.733886 & 0.25 & 12.6 \\
\hline J182943.52-280931.5 & $18: 29: 43.52$ & $-28: 09: 31.5$ & 5.374 & -8.103 & 14.417 & 0.512792 & 0.29 & 9.4 \\
\hline J182948.34-312426.7 & $18: 29: 48.34$ & $-31: 24: 26.7$ & 2.434 & -9.557 & 13.2 & & & 7.0 \\
\hline J182950.65-28 & & $-28: 25: 01.1$ & 5.152 & -8.242 & 14.5 & & 0.2 & 10.9 \\
\hline J182951.59-304539.5 & $18: 29: 51.59$ & $-30: 45: 39.5$ & 3.027 & -9.283 & 14.135 & 0.583284 & 0.36 & 8.8 \\
\hline $\mathrm{J} 18295$ & 51.68 & 5:06.1 & 2.732 & -9.426 & 13.413 & 0.861943 & 0.26 & 7.7 \\
\hline J182954.03-310517.6* & $18: 29: 54.03$ & $-31: 05: 17.6$ & 2.733 & -9.435 & 13.884 & 0.478565 & 0.34 & 7.0 \\
\hline J182954.34-293903.5 & $18: 29: 54.34$ & $-29: 39: 03.5$ & 4.040 & -8.802 & 14.052 & 0.565368 & 0.29 & 8.3 \\
\hline $\mathrm{J} 183003.83-284318.0$ & $18: 30: 03.83$ & $-28: 43: 18.0$ & 4.898 & -8.421 & 14.403 & 0.654420 & 0.28 & 10.7 \\
\hline J183005.63-303944.9 & $18: 30: 05.63$ & $-30: 39: 44.9$ & 3.139 & -9.285 & 13.913 & 0.565723 & 0.35 & 7.8 \\
\hline J183009.43-290306.2 & 18:30:09.43 & $-29: 03: 06.2$ & 4.608 & -8.586 & 14.316 & 0.560808 & 0.38 & 9.4 \\
\hline $\mathrm{J} 183010.90-323112.6 *$ & $18: 30: 10.90$ & $-32: 31: 12.6$ & 1.453 & -10.116 & 14.389 & 0.598483 & 0.26 & 10.1 \\
\hline J183011.13-290410.1 & $18: 30: 11.13$ & $-29: 04: 10.1$ & 4.595 & -8.599 & 14.244 & 0.578700 & 0.25 & 9.2 \\
\hline J183013.78-291717.9 & $18: 30: 13.78$ & $-29: 17: 17.9$ & 4.401 & -8.705 & 14.778 & 0.448593 & 0.40 & 10.4 \\
\hline J183016.58-284047.4 & $18: 30: 16.58$ & $-28: 40: 47.4$ & 4.957 & -8.444 & 14.917 & 0.518850 & 0.27 & 12.1 \\
\hline $\mathrm{J} 183016.66-283514.5 *$ & $18: 30: 16.66$ & $-28: 35: 14.5$ & & & & & & 6.5 \\
\hline J183021.35-294827.5 & $18: 30: 21.35$ & $-29: 48: 27.5$ & 3.942 & -8.959 & 15.053 & 0.463678 & 0.26 & 12.1 \\
\hline $\mathrm{J} 183022.51-310555.8$ & $18: 30: 22.51$ & $-31: 05: 55.8$ & 2.769 & -9.531 & 14.998 & 0.441312 & 0.25 & 11.5 \\
\hline $\mathrm{J} 183025.64-305556.1 *$ & $18: 30: 25.64$ & $-30: 55: 56.1$ & 2.926 & -9.468 & 14.392 & 0.448175 & 0.26 & 8.6 \\
\hline J183026.50-281016.7 & $18: 30: 26.50$ & $-28: 10: 16.7$ & 5.433 & -8.250 & 14.582 & 0.547339 & 0.30 & 10.6 \\
\hline $\mathrm{J} 183027.57-300103.9 *$ & $18: 30: 27.57$ & $-30: 01: 03.9$ & 3.761 & -9.072 & 13.414 & 0.419562 & 0.22 & 5.2 \\
\hline J183028.22-283518.7 & $18: 30: 28.22$ & $-28: 35: 18.7$ & 5.059 & -8.441 & 14.124 & 0.473981 & 0.36 & 7.8 \\
\hline J183030.86-294823.7 & $18: 30: 30.86$ & $-29: 48: 23.7$ & 3.958 & -8.989 & 14.846 & 0.456486 & 0.31 & 10.9 \\
\hline J183034.09-285655.7 & 18:30:34.09 & $-28: 56: 55.7$ & 4.742 & -8.621 & 15.136 & 0.535667 & 0.24 & 13.6 \\
\hline J183034.83-310409.1 & $18: 30: 34.83$ & $-31: 04: 09.1$ & 2.815 & -9.558 & 14.284 & 0.520313 & 0.28 & 8.9 \\
\hline J183037.40-303115.2 & $18: 30: 37.40$ & $-30: 31: 15.2$ & 3.319 & -9.325 & 13.973 & 0.509980 & 0.25 & 7.6 \\
\hline J183038.43-291811.5 & $18: 30: 38.43$ & $-29: 18: 11.5$ & 4.427 & -8.792 & 14.197 & 0.462299 & 0.31 & 8.0 \\
\hline
\end{tabular}


Table A.1. continued.

\begin{tabular}{|c|c|c|c|c|c|c|c|c|}
\hline VVV ID & $\begin{array}{l}\text { RA (J2000.0) } \\
\text { hh:mm:ss.ss }\end{array}$ & $\begin{array}{c}\text { Dec (J2000.0) } \\
\text { dd:mm:ss.s }\end{array}$ & $\begin{array}{c}\ell \\
(\mathrm{deg})\end{array}$ & $\begin{array}{c}b \\
(\mathrm{deg})\end{array}$ & $\begin{array}{l}\left\langle K_{\mathrm{s}}\right\rangle \\
(\mathrm{mag})\end{array}$ & $\begin{array}{c}P \\
\text { (days) }\end{array}$ & $\begin{array}{l}\text { Amplitude } \\
\text { (mag) }\end{array}$ & $\begin{array}{c}d \\
(\mathrm{kpc})\end{array}$ \\
\hline $\mathrm{J} 183045.33-322237.8 *$ & $18: 30: 45.33$ & $-32: 22: 37.8$ & 1.638 & -10.162 & 14.691 & 0.490333 & 0.34 & 10.5 \\
\hline J183048.73-310954.4* & $18: 30: 48.73$ & $-31: 09: 54.4$ & 2.750 & -9.644 & 14.274 & 0.491565 & 0.35 & 8.6 \\
\hline J183051.28-320058.4 & $18: 30: 51.28$ & $-32: 00: 58.4$ & 1.977 & -10.024 & 14.302 & 0.549064 & 0.28 & 9.2 \\
\hline J183054.75-292227.6 & 18:30:54.75 & $-29: 22: 27.6$ & 4.389 & -8.876 & 14.108 & 0.578777 & 0.30 & 8.6 \\
\hline J183055.00-285232.1 & 18:30:55.00 & $-28: 52: 32.1$ & 4.842 & -8.656 & 14.646 & 0.698880 & 0.28 & 12.4 \\
\hline $\mathrm{J} 183056.63-323329.3$ & $18: 30: 56.63$ & $-32: 33: 29.3$ & 1.490 & -10.277 & 14.543 & 0.624707 & 0.24 & 11.1 \\
\hline J183059.06-285330.6 & $18: 30: 59.06$ & $-28: 53: 30.6$ & 4.834 & -8.677 & 14.322 & 0.506598 & 0.30 & 8.9 \\
\hline $\mathrm{J} 183100.49-315343.2$ & 18:31:00.49 & $-31: 53: 43.2$ & 2.102 & -10.001 & 14.139 & 0.392082 & 0.34 & 7.1 \\
\hline J183101.91-274613.8 & 18:31:01.91 & $-27: 46: 13.8$ & 5.855 & -8.189 & 13.549 & 0.658814 & & 7.1 \\
\hline $\mathrm{J} 183104.34-321729.7 *$ & $18: 31: 04.34$ & $-32: 17: 29.7$ & 1.745 & -10.185 & 15.298 & 0.474944 & & 13.8 \\
\hline J183108.75-320339.2 & 18:31:08.75 & $-32: 03: 39.2$ & 1.963 & -10.099 & 14.230 & 0.688231 & 0.30 & 10.1 \\
\hline J183108.87-285835.5 & $18: 31: 08.87$ & $-28: 58: 35.5$ & 4.773 & -8.746 & 14.825 & 0.533058 & 35 & 11.7 \\
\hline $\mathrm{J} 183108.87-304114.9$ & $18: 31: 08.87$ & $-30: 41: 14.9$ & 3.217 & -9.500 & 14.235 & 0.550694 & .27 & 9.0 \\
\hline J183109.14-303201.9 & 18:31:09.14 & $-30: 32: 01.9$ & 3.358 & -9.433 & 14.632 & 0.429954 & 0.30 & 9.5 \\
\hline $\mathrm{J} 183111.89-314114.2 *$ & 18:31:11.89 & $-31: 41: 14.2$ & 2.310 & -9.946 & 14.263 & 0.635292 & 0.29 & 9.8 \\
\hline $\mathrm{J} 183112.60-314441.3 *$ & $18: 31: 12.60$ & $-31: 44: 41.3$ & 2.258 & -9.974 & 14.875 & 0.743810 & 0.28 & 14.4 \\
\hline J183113.14-272928.2 & $18: 31: 13.14$ & $-27: 29: 28.2$ & 6.126 & -8.102 & 14.179 & 0.477213 & 0.34 & 8.1 \\
\hline J183114.34-275222.7 & $18: 31: 14.34$ & $-27: 52: 22.7$ & 5.783 & -8.275 & 13.870 & 0.682715 & 0.30 & 8.4 \\
\hline J183117.20-303841.9 & 18:31:17.20 & $-30: 38: 41.9$ & 3.269 & -9.508 & 14.250 & 0.555596 & 0.31 & 9.1 \\
\hline J183118.16-302610.9 & $18: 31: 18.16$ & $-30: 26: 10.9$ & 3.461 & -9.420 & 15.637 & 0.524742 & 0.29 & 17.2 \\
\hline $\mathrm{J} 183127.38-312529.0$ & $18: 31: 27.38$ & $-31: 25: 29.0$ & 2.574 & -9.881 & 13.859 & 0.549583 & 0.23 & 7.5 \\
\hline J183130.82-310906.1 & $18: 31: 30.82$ & $-31: 09: 06.1$ & 2.829 & -9.773 & 14.7 & 0.5414 & & 11.6 \\
\hline $\mathrm{J} 183135.26-282845.5$ & $18: 31: 35.26$ & $-28: 28: 45.5$ & 5.268 & -8.613 & 15.516 & 0.477546 & & 15.4 \\
\hline J183139.79-291340.0 & $18: 31: 39.79$ & $-29: 13: 40.0$ & 4.595 & -8.958 & 14.330 & 0.427241 & & 8.2 \\
\hline $42.15-271014.3$ & $18: 3$ & $: 14.3$ & 6.464 & -8.055 & 14.012 & 0.680 & 31 & 9.0 \\
\hline J183142.46-275158.3 & $18: 31$ & $-27: 51: 58.3$ & 5.835 & -8.365 & 14.354 & 0.608280 & 0.24 & 10.0 \\
\hline $\mathrm{J} 183143.82-310912.2$ & 3.82 & $-31: 09: 12.2$ & 2.848 & -9.816 & 13.567 & 0.614726 & 0.29 & 6.9 \\
\hline J183143.93-290401.4 & $18: 31: 43.93$ & $-29: 04: 01.4$ & 4.748 & -8.901 & 14.073 & 0.457 & 0.33 & 7.5 \\
\hline $\mathrm{J} 183145.02-302244.0$ & $18: 31: 45.02$ & $-30: 22: 44.0$ & 3.556 & -9.481 & 13.832 & 0.593257 & 0.32 & 7.7 \\
\hline J183146.55-280021.1 & $18: 31: 46.55$ & $-28: 00: 21.1$ & 5.715 & -8.441 & 13.360 & 0.818357 & 0.24 & 7.3 \\
\hline $\mathrm{J} 183149.86-311535.4 *$ & $18: 31: 49.86$ & $-31: 15: 35.4$ & 2.760 & -9.882 & 13.786 & 0.584505 & 0.30 & 7.4 \\
\hline J183150.02-272911.1 & 18:31:50.02 & $-27: 29: 11.1$ & 6.192 & -8.222 & 14.310 & 0.601417 & 0.34 & 9.7 \\
\hline J183155.75-280031.7 & $18: 31: 55.75$ & $-28: 00: 31.7$ & 5.728 & -8.472 & 15.372 & 0.516097 & 0.34 & 15.0 \\
\hline $\mathrm{J} 183159.32-281402.2$ & $18: 31: 59.32$ & $-28: 14: 02.2$ & 5.530 & -8.584 & 14.507 & 0.489275 & 0.34 & 9.6 \\
\hline $\mathrm{J} 183200.43-304608.2$ & $18: 32: 00.43$ & $-30: 46: 08.2$ & 3.225 & -9.701 & 14.729 & 0.767371 & 0.22 & 13.6 \\
\hline J183204.38-294047.5 & 18:32:04.38 & $-29: 40: 47.5$ & 4.224 & -9.237 & 15.0 & & & 12.2 \\
\hline $11.63-291936.9$ & & $-29: 19: 3$ & & -9.106 & 14.3 & & & 9.2 \\
\hline J183217.84-281643.3 & $18: 32: 17.84$ & $-28: 16: 43.3$ & 5.519 & -8.664 & 13.862 & 0.553983 & 0.21 & 7.5 \\
\hline J183217.85-290824.1 & 17.85 & $-29: 08: 24.1$ & 4.737 & -9.044 & 14.964 & 0.526072 & 0.27 & 12.4 \\
\hline J183220.66-295731.3 & 20.66 & $-29: 57: 31.3$ & 3.996 & -9.413 & 14.533 & 0.545464 & 0.23 & 10.3 \\
\hline $\mathrm{J} 183221.31-281514.8$ & $18: 32: 21.31$ & $-28: 15: 14.8$ & 5.547 & -8.665 & 14.123 & 0.465731 & 0.32 & 7.7 \\
\hline J183223.20-301859.2 & $18: 32: 23.20$ & $-30: 18: 59.2$ & 3.674 & -9.577 & 14.255 & 0.550903 & 0.22 & 9.0 \\
\hline $\mathrm{J} 183230.45-302800.7$ & $18: 32: 30.45$ & $-30: 28: 00.7$ & 3.548 & -9.666 & 14.549 & 0.431090 & 0.31 & 9.1 \\
\hline $\mathrm{J} 183232.37-310924.2 *$ & $18: 32: 32.37$ & $-31: 09: 24.2$ & 2.921 & -9.973 & 13.716 & 0.552746 & 0.31 & 7.0 \\
\hline J183234.91-312018.5* & $18: 32: 34.91$ & $-31: 20: 18.5$ & 2.759 & -10.06 & 13.403 & 0.472979 & 0.26 & 5.5 \\
\hline $\mathrm{J} 183240.12-313547.4 *$ & $18: 32: 40.12$ & $-31: 35: 47.4$ & 2.531 & -10.189 & 13.834 & 0.549556 & 0.33 & 7.4 \\
\hline J183240.57-311648.1 & $18: 32: 40.57$ & $-31: 16: 48.1$ & 2.821 & -10.053 & 13.261 & 0.678348 & 0.21 & 6.3 \\
\hline J183243.41-310142.7 & $18: 32: 43.41$ & $-31: 01: 42.7$ & 3.056 & -9.953 & 13.879 & 0.803200 & 0.17 & 9.2 \\
\hline J183245.79-304813.1 & $18: 32: 45.79$ & $-30: 48: 13.1$ & 3.26 & -9.8 & & & & 8.5 \\
\hline J183251.66-293858.2 & $18: 32: 51.66$ & $-29: 38: 58.2$ & 4.328 & -9.378 & 14.378 & 0.497673 & 0.34 & 9.1 \\
\hline J183253.51-272737.5 & $18: 32: 53.51$ & $-27: 27: 37.5$ & 6.320 & -8.420 & 15.191 & 0.545200 & 0.30 & 14.1 \\
\hline J183255.64-274735.8 & $18: 32: 55.64$ & $-27: 47: 35.8$ & 6.022 & -8.575 & 14.277 & 0.543889 & 0.26 & 9.1 \\
\hline $\mathrm{J} 183255.91-312612.9 *$ & $18: 32: 55.91$ & $-31: 26: 12.9$ & 2.702 & -10.170 & 14.731 & 0.516177 & 0.32 & 11.0 \\
\hline J183256.52-291057.2 & $18: 32: 56.52$ & $-29: 10: 57.2$ & 4.761 & -9.189 & 14.508 & 0.573763 & 0.30 & 10.4 \\
\hline J183257.86-310942.3 & $18: 32: 57.86$ & $-31: 09: 42.3$ & 2.957 & -10.057 & 13.193 & 0.714531 & 0.27 & 6.2 \\
\hline J183259.83-270500.9 & $18: 32: 59.83$ & $-27: 05: 00.9$ & 6.672 & -8.274 & 14.313 & 0.552364 & 0.33 & 9.3 \\
\hline J183300.69-270500.9 & 18:33:00.69 & $-27: 05: 00.9$ & 6.674 & -8.277 & 14.283 & 0.616333 & 0.23 & 9.7 \\
\hline $\mathrm{J} 183302.25-274314.3$ & 18:33:02.25 & $-27: 43: 14.3$ & 6.099 & -8.564 & 15.255 & 0.557432 & 0.31 & 14.8 \\
\hline J183302.59-304209.0 & 18:33:02.59 & $-30: 42: 09.0$ & 3.384 & -9.873 & 14.887 & 0.529911 & 0.33 & 12.0 \\
\hline J183305.31-294625.5 & 18:33:05.31 & $-29: 46: 25.5$ & 4.236 & -9.477 & 15.095 & 0.511491 & 0.32 & 13.0 \\
\hline
\end{tabular}


Table A.1. continued.

\begin{tabular}{|c|c|c|c|c|c|c|c|c|}
\hline VVV ID & $\begin{array}{c}\text { RA (J2000.0) } \\
\text { hh:mm:ss.ss }\end{array}$ & $\begin{array}{c}\text { Dec (J2000.0) } \\
\text { dd:mm:ss.s }\end{array}$ & $\begin{array}{c}\ell \\
\text { (deg) }\end{array}$ & $\begin{array}{c}b \\
(\mathrm{deg})\end{array}$ & $\begin{array}{c}\left\langle K_{\mathrm{s}}\right\rangle \\
(\mathrm{mag})\end{array}$ & $\begin{array}{c}P \\
\text { (days) }\end{array}$ & $\begin{array}{l}\text { Amplitude } \\
\text { (mag) }\end{array}$ & $\begin{array}{c}d \\
(\mathrm{kpc})\end{array}$ \\
\hline J183307.09-305707.5 & 18:33:07.09 & $-30: 57: 07.5$ & 3.163 & -9.996 & 14.411 & 0.521722 & 0.24 & 9.5 \\
\hline J183308.14-294313.4 & 18:33:08.14 & $-29: 43: 13.4$ & 4.290 & -9.463 & 14.784 & 0.489764 & 0.32 & 11.0 \\
\hline J183310.92-285537.8 & $18: 33: 10.92$ & $-28: 55: 37.8$ & 5.017 & -9.124 & 14.227 & 0.643360 & 0.28 & 9.7 \\
\hline $\mathrm{J} 183317.23-264230.6$ & $18: 33: 17.23$ & $-26: 42: 30.6$ & 7.041 & -8.166 & 14.440 & 0.549345 & 0.29 & 9.9 \\
\hline J183317.54-283543.8 & $18: 33: 17.54$ & $-28: 35: 43.8$ & 5.329 & -9.000 & 14.733 & 0.574082 & 0.26 & 11.6 \\
\hline J183320.37-293233.8 & $18: 33: 20.37$ & $-29: 32: 33.8$ & 4.471 & -9.425 & 14.814 & 0.477637 & 0.40 & 11.0 \\
\hline J183323.16-264255.1 & $18: 33: 23.16$ & $-26: 42: 55.1$ & 7.045 & -8.189 & 13.898 & 0.556929 & 0.25 & 7.7 \\
\hline J183329.46-265556.3 & $18: 33: 29.46$ & $-26: 55: 56.3$ & 6.859 & -8.306 & 14.577 & 0.477158 & 0.32 & 9.8 \\
\hline J183330.94-300211.6 & $18: 33: 30.94$ & $-30: 02: 11.6$ & 4.038 & -9.675 & 14.349 & 0.565764 & 0.26 & 9.6 \\
\hline J183331.70-295124.4 & $18: 33: 31.70$ & $-29: 51: 24.4$ & 4.203 & -9.599 & 14.454 & 0.453579 & 0.30 & 9.0 \\
\hline J183336.62-304302.6 & $18: 33: 36.62$ & $-30: 43: 02.6$ & 3.424 & -9.989 & 14.153 & 0.475934 & 0.27 & 8.0 \\
\hline J183339.12-271619.3 & $18: 33: 39.12$ & $-27: 16: 19.3$ & 6.567 & -8.488 & 14.494 & 0.446630 & 0.37 & 9.1 \\
\hline J183339.91-291657.1 & $18: 33: 39.91$ & $-29: 16: 57.1$ & 4.740 & -9.375 & 14.375 & 0.554813 & 0.26 & 9.6 \\
\hline J183340.77-262621.4 & $18: 33: 40.77$ & $-26: 26: 21.4$ & 7.324 & -8.125 & 13.632 & 0.663064 & 0.30 & 7.4 \\
\hline J183346.56-305515.9 & $18: 33: 46.56$ & $-30: 55: 15.9$ & 3.254 & -10.109 & 14.215 & 0.572666 & 0.27 & 9.1 \\
\hline J183349.90-273848.9 & $18: 33: 49.90$ & $-27: 38: 48.9$ & 6.244 & -8.690 & 14.007 & 0.559425 & 0.29 & 8.1 \\
\hline $\mathrm{J} 183350.72-303700.4$ & $18: 33: 50.72$ & $-30: 37: 00.4$ & 3.539 & -9.991 & 14.664 & 0.401218 & 0.35 & 9.3 \\
\hline J183353.87-273040.6 & $18: 33: 53.87$ & $-27: 30: 40.6$ & 6.374 & -8.643 & 14.047 & 0.452798 & 0.18 & 7.4 \\
\hline $\mathrm{J} 183354.26-301027.8$ & $18: 33: 54.26$ & $-30: 10: 27.8$ & 3.949 & -9.810 & 14.315 & 0.643840 & 0.37 & 10.1 \\
\hline J183357.37-285558.7 & $18: 33: 57.37$ & $-28: 55: 58.7$ & 5.087 & -9.279 & 13.527 & 0.499555 & 0.30 & 6.0 \\
\hline J183357.86-291636.2 & $18: 33: 57.86$ & $-29: 16: 36.2$ & 4.774 & -9.431 & 14.544 & 0.431934 & 0.34 & 9.1 \\
\hline J183358.62-303613.5 & $18: 33: 58.62$ & $-30: 36: 13.5$ & 3.563 & -10.011 & 13.668 & 0.566739 & 0.32 & 6.9 \\
\hline $\mathrm{J} 183403.35-271603.2$ & $18: 34: 03.35$ & $-27: 16: 03.2$ & 6.611 & -8.567 & 14.199 & 0.520592 & 0.39 & 8.5 \\
\hline J183403.44-261031.5 & $18: 34: 03.44$ & $-26: 10: 31.5$ & 7.601 & -8.084 & 14.187 & 0.456886 & 0.34 & 7.9 \\
\hline J183406.43-275509.2 & $18: 34: 06.43$ & $-27: 55: 09.2$ & 6.024 & -8.864 & 13.652 & 0.648433 & 0.27 & 7.4 \\
\hline J183406.84-282111.3 & $18: 34: 06.84$ & $-28: 21: 11.3$ & 5.630 & -9.056 & 14.201 & 0.659387 & 0.30 & 9.7 \\
\hline J183415.06-285924.7 & $18: 34: 15.06$ & $-28: 59: 24.7$ & 5.063 & -9.362 & 14.832 & 0.482868 & 0.38 & 11.1 \\
\hline J183418.19-280737.2 & $18: 34: 18.19$ & $-28: 07: 37.2$ & 5.854 & -8.994 & 13.990 & 0.589057 & 0.24 & 8.2 \\
\hline J183419.46-293532.9 & $18: 34: 19.46$ & $-29: 35: 32.9$ & 4.521 & -9.639 & 13.354 & 0.692852 & 0.31 & 6.6 \\
\hline J183420.99-295104.5 & $18: 34: 20.99$ & $-29: 51: 04.5$ & 4.287 & -9.757 & 13.691 & 0.742975 & 0.30 & 8.1 \\
\hline J183421.43-262902.6 & $18: 34: 21.43$ & $-26: 29: 02.6$ & 7.351 & -8.281 & 14.221 & 0.571759 & 0.25 & 9.1 \\
\hline J183422.19-285454.1 & $18: 34: 22.19$ & $-28: 54: 54.1$ & 5.143 & -9.353 & 14.303 & 0.466057 & 0.26 & 8.5 \\
\hline J183429.88-282753.6 & $18: 34: 29.88$ & $-28: 27: 53.6$ & 5.566 & -9.181 & 13.726 & 0.456608 & 0.33 & 6.3 \\
\hline J183430.19-273715.5 & $18: 34: 30.19$ & $-27: 37: 15.5$ & 6.334 & -8.812 & 13.290 & 0.664531 & 0.28 & 6.3 \\
\hline $\mathrm{J} 183432.82-262329.3$ & $18: 34: 32.82$ & $-26: 23: 29.3$ & 7.454 & -8.278 & 13.993 & 0.539095 & 0.22 & 7.9 \\
\hline J183434.61-263624.5 & $18: 34: 34.61$ & $-26: 36: 24.5$ & 7.262 & -8.380 & 14.324 & 0.566049 & 0.24 & 9.5 \\
\hline J183436.96-295836.1 & $18: 34: 36.96$ & $-29: 58: 36.1$ & 4.197 & -9.863 & 14.967 & 0.627837 & 0.30 & 13.7 \\
\hline J183439.69-275612.1 & $18: 34: 39.69$ & $-27: 56: 12.1$ & 6.062 & -8.982 & 14.810 & 0.448815 & 0.21 & 10.6 \\
\hline J183441.36-255504.9 & $18: 34: 41.36$ & $-25: 55: 04.9$ & 7.897 & -8.097 & 14.539 & 0.606487 & 0.27 & 10.9 \\
\hline J183444.28-283746.1 & $18: 34: 44.28$ & $-28: 37: 46.1$ & 5.439 & -9.300 & 14.194 & 0.500153 & 0.29 & 8.3 \\
\hline J183445.11-295804.3 & $18: 34: 45.11$ & $-29: 58: 04.3$ & 4.218 & -9.886 & 14.184 & 0.474032 & 0.26 & 8.1 \\
\hline J183446.57-294358.1 & $18: 34: 46.57$ & $-29: 43: 58.1$ & 4.436 & -9.789 & 14.360 & 0.608543 & 0.21 & 10.0 \\
\hline $\mathrm{J} 183447.68-272020.2$ & $18: 34: 47.68$ & $-27: 20: 20.2$ & 6.619 & -8.746 & 14.253 & 0.628674 & 0.18 & 9.7 \\
\hline J183451.76-280735.9 & $18: 34: 51.76$ & $-28: 07: 35.9$ & 5.909 & -9.105 & 14.098 & 0.533439 & 0.24 & 8.2 \\
\hline J183457.98-273947.0 & $18: 34: 57.98$ & $-27: 39: 47.0$ & 6.341 & -8.922 & 13.911 & 0.543030 & 0.22 & 7.6 \\
\hline $\mathrm{J} 183502.27-272617.7$ & $18: 35: 02.27$ & $-27: 26: 17.7$ & 6.553 & -8.838 & 15.524 & 0.617577 & 0.32 & 17.8 \\
\hline J183509.57-295734.4 & $18: 35: 09.57$ & $-29: 57: 34.4$ & 4.265 & -9.962 & 14.890 & 0.530217 & 0.28 & 12.0 \\
\hline $\mathrm{J} 183509.77-274702.3$ & 18:35:09.77 & $-27: 47: 02.3$ & 6.251 & -9.014 & 14.259 & 0.571719 & 0.34 & 9.2 \\
\hline $\mathrm{J} 183511.21-274402.4$ & $18: 35: 11.21$ & $-27: 44: 02.4$ & 6.298 & -8.997 & 14.724 & 0.435724 & 0.37 & 10.0 \\
\hline J183511.48-295741.8 & $18: 35: 11.48$ & $-29: 57: 41.8$ & 4.266 & -9.969 & 13.782 & 0.626833 & 0.30 & 7.7 \\
\hline J183515.08-274151.2 & $18: 35: 15.08$ & $-27: 41: 51.2$ & 6.338 & -8.994 & 15.382 & 0.507344 & 0.34 & 14.9 \\
\hline J183518.04-275113.6 & $18: 35: 18.04$ & $-27: 51: 13.6$ & 6.201 & -9.072 & 13.956 & 0.749968 & 0.23 & 9.2 \\
\hline $\mathrm{J} 183519.42-270140.2$ & $18: 35: 19.42$ & $-27: 01: 40.2$ & 6.954 & -8.715 & 14.552 & 0.484611 & 0.27 & 9.7 \\
\hline J183520.98-264403.7 & $18: 35: 20.98$ & $-26: 44: 03.7$ & 7.223 & -8.591 & 12.774 & 0.570321 & 0.28 & 4.5 \\
\hline J183521.33-273504.6 & $18: 35: 21.33$ & $-27: 35: 04.6$ & 6.451 & -8.965 & 14.055 & 0.694758 & 0.27 & 9.3 \\
\hline J183522.29-255628.6 & $18: 35: 22.29$ & $-25: 56: 28.6$ & 7.945 & -8.245 & 14.505 & 0.433903 & 0.34 & 9.0 \\
\hline J183523.79-290434.4 & $18: 35: 23.79$ & $-29: 04: 34.4$ & 5.095 & -9.625 & 13.803 & 0.532484 & 0.26 & 7.1 \\
\hline $\mathrm{J} 183531.73-265000.3$ & $18: 35: 31.73$ & $-26: 50: 00.3$ & 7.151 & -8.670 & 14.784 & 0.590479 & 0.30 & 12.1 \\
\hline J183531.85-255505.1 & $18: 35: 31.85$ & $-25: 55: 05.1$ & 7.982 & -8.267 & 15.098 & 0.522316 & 0.34 & 13.2 \\
\hline J183532.11-270932.9 & $18: 35: 32.11$ & $-27: 09: 32.9$ & 6.856 & -8.815 & 14.331 & 0.511091 & 0.24 & 9.0 \\
\hline
\end{tabular}


Table A.1. continued.

\begin{tabular}{|c|c|c|c|c|c|c|c|c|}
\hline VVV ID & $\begin{array}{c}\text { RA (J2000.0) } \\
\text { hh:mm:ss.ss }\end{array}$ & $\begin{array}{c}\text { Dec (J2000.0) } \\
\text { dd:mm:ss.s }\end{array}$ & $\begin{array}{c}\ell \\
(\mathrm{deg})\end{array}$ & $\begin{array}{c}b \\
(\mathrm{deg})\end{array}$ & $\begin{array}{l}\left\langle K_{\mathrm{s}}\right\rangle \\
(\mathrm{mag})\end{array}$ & $\begin{array}{c}P \\
\text { (days) }\end{array}$ & $\begin{array}{l}\text { Amplitude } \\
\text { (mag) }\end{array}$ & $\begin{array}{c}d \\
(\mathrm{kpc})\end{array}$ \\
\hline J183532.33-264853.6 & $18: 35: 32.33$ & $-26: 48: 53.6$ & 7.169 & -8.664 & 14.838 & 0.490952 & 0.42 & 11.3 \\
\hline J183536.59-271717.0 & $18: 35: 36.59$ & $-27: 17: 17.0$ & 6.746 & -8.886 & 13.882 & 0.494130 & 0.33 & 7.1 \\
\hline J183538.81-270026.8 & $18: 35: 38.81$ & $-27: 00: 26.8$ & 7.005 & -8.770 & 14.268 & 0.501819 & 0.33 & 8.6 \\
\hline J183540.25-282947.5 & $18: 35: 40.25$ & $-28: 29: 47.5$ & 5.651 & -9.427 & 15.527 & 0.524981 & 0.37 & 16.3 \\
\hline $\mathrm{J} 183549.58-265310.4$ & $18: 35: 49.58$ & $-26: 53: 10.4$ & 7.133 & -8.753 & 13.977 & 0.531082 & 0.29 & 7.7 \\
\hline J183551.71-280526.9 & $18: 35: 51.71$ & $-28: 05: 26.9$ & 6.039 & -9.288 & 14.130 & 0.534291 & & 8.4 \\
\hline J183552.08-283355.0 & $18: 35: 52.08$ & $-28: 33: 55.0$ & 5.607 & -9.496 & 14.608 & 0.633413 & 0.26 & 11.6 \\
\hline J183553.52-285226.5 & $18: 35: 53.52$ & $-28: 52: 26.5$ & 5.328 & -9.635 & 13.439 & 0.473979 & 0.25 & 5.6 \\
\hline J183555.23-264001.0 & $18: 35: 55.23$ & $-26: 40: 01.0$ & 7.341 & -8.676 & 13.850 & 0.561337 & 0.31 & 7.5 \\
\hline J183557.69-300435.0 & $18: 35: 57.69$ & $-30: 04: 35.0$ & 4.234 & -10.169 & 14.852 & 0.633770 & 0.28 & 13.0 \\
\hline J183559.35-253902.2 & $18: 35: 59.35$ & $-25: 39: 02.2$ & 8.270 & -8.242 & 14.316 & 0.540856 & 0.23 & 9.2 \\
\hline J183559.48-255209.2 & $18: 35: 59.48$ & $-25: 52: 09.2$ & 8.072 & -8.339 & 14.498 & 0.539820 & 0.34 & 10.1 \\
\hline J183603.14-274241.1 & $18: 36: 03.14$ & $-27: 42: 41.1$ & 6.404 & -9.160 & 14.468 & 0.463633 & 0.34 & 9.1 \\
\hline J183603.36-251259.9 & $18: 36: 03.36$ & $-25: 12: 59.9$ & 8.670 & -8.063 & 14.639 & 0.760104 & 0.25 & 13.0 \\
\hline J183604.22-285849.9 & $18: 36: 04.22$ & $-28: 58: 49.9$ & 5.248 & -9.716 & 13.509 & 0.454806 & 0.36 & 5.7 \\
\hline J183607.93-254444.4 & 18:36:07.93 & $-25: 44: 44.4$ & 8.199 & -8.313 & 14.215 & 0.601728 & 0.31 & 9.3 \\
\hline J183610.30-300015.5 & $18: 36: 10.30$ & $-30: 00: 15.5$ & 4.320 & -10.179 & 15.631 & 0.728051 & & 20.5 \\
\hline J183615.30-282749.6 & $18: 36: 15.30$ & $-28: 27: 49.6$ & 5.737 & -9.528 & 14.541 & 0.548 & & 10.4 \\
\hline J183622.86-255419.0 & $18: 36: 22.86$ & $-25: 54: 19.0$ & 8.079 & -8.4 & 14.176 & 0.632 & & 9.4 \\
\hline J183623.19-273727.3 & $18: 36: 23.19$ & $-27: 37: 27.3$ & 6.516 & -9.188 & 14.202 & 0.498331 & 0.25 & 8.3 \\
\hline J183630.19-260416.8 & $18: 36: 30.19$ & $-26: 04: 16.8$ & 7.940 & -8.531 & 14.545 & 0.635538 & 0.33 & 11.2 \\
\hline J183631.12-282642.3 & $18: 36: 31.12$ & $-28: 26: 42.3$ & 5.780 & -9.572 & 14.320 & 0.446445 & 0.25 & 8.3 \\
\hline J183632.74-274934.1 & $18: 36: 32.74$ & $-27: 49: 34.1$ & 6.348 & -9.308 & 14.602 & 0.580659 & 0.25 & 11.0 \\
\hline J183633.32-261812.5 & $18: 36: 33.32$ & $-26: 18: 12.5$ & 7.735 & -8.643 & 13.744 & 0.551794 & 0.29 & 7.1 \\
\hline J183637.80-261326.3 & $18: 36: 37.80$ & $-26: 13: 26.3$ & 7.814 & -8.624 & 13.975 & 0.652197 & 0.33 & 8.6 \\
\hline J183639.37-263830.5 & $18: 36: 39.37$ & $-26: 38: 30.5$ & 7.437 & -8.812 & 14.940 & 0.616314 & 0.32 & 13.4 \\
\hline J183641.34-295240.6 & $18: 36: 41.34$ & $-29: 52: 40.6$ & 4.485 & -10.225 & 15.371 & 0.587979 & 0.34 & 16.1 \\
\hline J183642.73-285702.3 & $18: 36: 42.73$ & $-28: 57: 02.3$ & 5.337 & -9.830 & 14.438 & 0.613279 & 0.29 & 10.5 \\
\hline J183645.95-290719.9 & $18: 36: 45.95$ & $-29: 07: 19.9$ & 5.185 & -9.914 & 14.260 & 0.496818 & 0.22 & 8.6 \\
\hline J183646.44-260949.1 & $18: 36: 46.44$ & $-26: 09: 49.1$ & 7.883 & -8.626 & 14.138 & 0.651479 & & 9.4 \\
\hline J183647.27-271023.3 & $18: 36: 47.27$ & $-27: 10: 23.3$ & 6.966 & -9.071 & 13.861 & 0.679750 & & 8.4 \\
\hline J183648.96-283002.5 & $18: 36: 48.96$ & $-28: 30: 02.5$ & 5.758 & -9.655 & 14.431 & & & 9.5 \\
\hline J183650.56-283053.6 & & $-28: 30: 53.6$ & & -9.666 & & & & 6.5 \\
\hline J1836 & $18: 36$ & $-27:$ & 6.489 & -9.318 & 14.623 & 0.50 & 0.34 & 10.3 \\
\hline J183652.36-291045.1* & $18: 36: 52.36$ & $-29: 10: 45.1$ & 5.143 & -9.960 & 14.018 & 0.507056 & 0.31 & 7.7 \\
\hline J183652.69-263438.9 & $18: 36: 52.69$ & $-26: 34: 38.9$ & 7.518 & -8.829 & 14.228 & 0.625168 & 0.18 & 9.6 \\
\hline J183657.71-293355.0 & $18: 36: 57.71$ & $-29: 33: 55.0$ & 4.798 & -10.144 & 14.155 & 0.446674 & 0.29 & 7.7 \\
\hline J183704.40-280207.6 & $18: 37: 04.40$ & $-28: 02: 07.6$ & 6.208 & -9.504 & 14.901 & 0.568253 & 0.28 & 12.6 \\
\hline J183706.09-270025.5 & $18: 37: 06.09$ & $-27: 00: 25.5$ & 7.149 & -9.061 & 13.844 & 0.563276 & 0.31 & 7.5 \\
\hline J183720.13-244102.7 & $18: 37: 20.13$ & $-24: 41: 02.7$ & 9.282 & -8.088 & 14.421 & 0.445665 & 0.40 & 8.7 \\
\hline J183721.62-244030.9 & $18: 37: 21.62$ & $-24: 40: 30.9$ & 9.293 & -8.089 & 14.494 & 0.474280 & 0.34 & 9.4 \\
\hline J183721.98-261507.8 & $18: 37: 21.98$ & $-26: 15: 07.8$ & 7.862 & -8.784 & 14.358 & 0.495955 & 0.33 & 9.0 \\
\hline J183724.25-291959.0 & $18: 37: 24.25$ & $-29: 19: 59.0$ & 5.053 & -10.131 & 13.979 & 0.543472 & 0.29 & 7.9 \\
\hline J183728.91-280519.1 & $18: 37: 28.91$ & $-28: 05: 19.1$ & 6.199 & -9.608 & 14.287 & 0.538407 & 0.2 & 9.1 \\
\hline $\mathrm{J} 183731.20-245034.5$ & $18: 37: 31.20$ & $-24: 50: 34.5$ & 9.157 & -8.196 & & 0.525100 & & 9.7 \\
\hline J183734.88-264207.7 & & & 7.474 & -9.0 & & & 0.2 & 8.7 \\
\hline J183739.34-263809.3 & $18: 37: 39.34$ & $-26: 38: 09.3$ & 7.541 & -9.010 & 14.421 & 0.597001 & 0.27 & 10.2 \\
\hline J183739.98-270929.9 & $18: 37: 39.98$ & $-27: 09: 29.9$ & 7.066 & -9.241 & 13.834 & 0.526222 & 0.29 & 7.2 \\
\hline J183742.09-261129.5 & $18: 37: 42.09$ & $-26: 11: 29.5$ & 7.950 & -8.825 & 14.114 & 0.626889 & 0.27 & 9.1 \\
\hline J183743.97-280637.3 & $18: 37: 43.97$ & $-28: 06: 37.3$ & 6.204 & -9.668 & 14.726 & 0.580171 & 0.33 & 11.7 \\
\hline J183746.16-281530.6* & $18: 37: 46.16$ & $-28: 15: 30.6$ & 6.072 & -9.739 & 12.484 & 0.616724 & 0.30 & 4.1 \\
\hline J183749.08-270132.8 & $18: 37: 49.08$ & $-27: 01: 32.8$ & 7.202 & -9.213 & 13.787 & 0.449214 & 0.30 & 6.5 \\
\hline J183751.34-273558.5 & $18: 37: 51.34$ & $-27: 35: 58.5$ & 6.682 & -9.470 & 13.562 & 0.643878 & 0.23 & 7.0 \\
\hline J183752.27-242843.7 & $18: 37: 52.27$ & $-24: 28: 43.7$ & 9.523 & -8.107 & 15.747 & 0.560620 & 0.36 & 18.8 \\
\hline J183752.43-241606.3 & $18: 37: 52.43$ & $-24: 16: 06.3$ & 9.714 & -8.015 & 13.582 & 0.655394 & 0.27 & 7.2 \\
\hline J183757.11-264651.1 & $18: 37: 57.11$ & $-26: 46: 51.1$ & 7.439 & -9.133 & 15.318 & 0.544695 & 0.25 & 15.0 \\
\hline J183758.37-272458.6 & $18: 37: 58.37$ & $-27: 24: 58.6$ & 6.861 & -9.414 & 13.706 & 0.588564 & 0.30 & 7.2 \\
\hline J183758.88-290835.4 & $18: 37: 58.88$ & $-29: 08: 35.4$ & 5.282 & -10.163 & 14.271 & 0.539540 & 0.36 & 9.0 \\
\hline J183804.05-283412.9 & 18:38:04.05 & $-28: 34: 12.9$ & 5.815 & -9.933 & 14.474 & 0.582992 & 0.29 & 10.4 \\
\hline J183804.26-242037.6 & 18:38:04.26 & $-24: 20: 37.6$ & 9.665 & -8.088 & 13.855 & 0.585929 & 0.30 & 7.7 \\
\hline
\end{tabular}


Table A.1. continued.

\begin{tabular}{|c|c|c|c|c|c|c|c|c|}
\hline VVV ID & $\begin{array}{c}\text { RA (J2000.0) } \\
\text { hh:mm:ss.ss }\end{array}$ & $\begin{array}{c}\text { Dec (J2000.0) } \\
\text { dd:mm:ss.s }\end{array}$ & $\begin{array}{c}\ell \\
(\mathrm{deg})\end{array}$ & $\begin{array}{c}b \\
(\mathrm{deg})\end{array}$ & $\begin{array}{c}\left\langle K_{\mathrm{s}}\right\rangle \\
(\mathrm{mag})\end{array}$ & $\begin{array}{c}P \\
\text { (days) }\end{array}$ & $\begin{array}{l}\text { Amplitude } \\
\text { (mag) }\end{array}$ & $\begin{array}{c}d \\
(\mathrm{kpc})\end{array}$ \\
\hline J183804.87-263637.9 & $18: 38: 04.87$ & $-26: 36: 37.9$ & 7.607 & -9.085 & 13.827 & 0.558887 & 0.27 & 7.4 \\
\hline J183805.70-285735.7 & 18:38:05.70 & $-28: 57: 35.7$ & 5.461 & -10.106 & 14.879 & 0.451048 & 0.39 & 11.0 \\
\hline J183806.30-275403.1 & 18:38:06.30 & $-27: 54: 03.1$ & 6.431 & -9.651 & 12.921 & 0.619721 & 0.25 & 5.1 \\
\hline J183807.96-251732.7 & 18:38:07.96 & $-25: 17: 32.7$ & 8.811 & -8.518 & 13.542 & 0.487524 & 0.32 & 6.0 \\
\hline J183808.34-255105.2 & 18:38:08.34 & $-25: 51: 05.2$ & 8.303 & -8.765 & 14.117 & 0.523390 & 0.25 & 8.2 \\
\hline J183808.50-251840.5 & 18:38:08.50 & $-25: 18: 40.5$ & 8.795 & -8.528 & 14.690 & 0.463188 & 0.33 & 10.2 \\
\hline J183809.59-274127.0* & 18:38:09.59 & $-27: 41: 27.0$ & 6.628 & -9.571 & 12.648 & 0.601971 & 0.31 & 4.4 \\
\hline J183811.49-254750.0 & $18: 38: 11.49$ & $-25: 47: 50.0$ & 8.358 & -8.752 & 14.071 & 0.471930 & 0.34 & 7.6 \\
\hline J183813.05-260150.1 & $18: 38: 13.05$ & $-26: 01: 50.1$ & 8.148 & -8.859 & 14.628 & 0.562107 & 0.31 & 10.9 \\
\hline J183821.06-262228.8 & $18: 38: 21.06$ & $-26: 22: 28.8$ & 7.848 & -9.036 & 14.378 & 0.568085 & 0.25 & 9.8 \\
\hline J183826.23-242232.8 & $18: 38: 26.23$ & $-24: 22: 32.8$ & 9.674 & -8.177 & 13.411 & 0.611090 & 0.15 & 6.4 \\
\hline J183828.67-271733.4 & $18: 38: 28.67$ & $-27: 17: 33.4$ & 7.023 & -9.461 & 14.306 & 0.525845 & 0.30 & 9.0 \\
\hline J183831.98-274415.4 & $18: 38: 31.98$ & $-27: 44: 15.4$ & 6.622 & -9.665 & 14.300 & 0.562933 & 0.27 & 9.3 \\
\hline J183837.59-270935.9 & $18: 38: 37.59$ & $-27: 09: 35.9$ & 7.159 & -9.434 & 14.768 & 0.451853 & 0.33 & 10.4 \\
\hline J183838.87-264954.3 & $18: 38: 38.87$ & $-26: 49: 54.3$ & 7.461 & -9.295 & 14.801 & 0.620151 & 0.34 & 12.6 \\
\hline J183840.44-282441.5 & $18: 38: 40.44$ & $-28: 24: 41.5$ & 6.019 & -9.985 & 14.457 & 0.497555 & 0.31 & 9.4 \\
\hline $\mathrm{J} 183844.77-250104.7$ & $18: 38: 44.77$ & $-25: 01: 04.7$ & 9.122 & -8.523 & 15.426 & 0.594781 & 0.26 & 16.6 \\
\hline $\mathrm{J} 183849.17-262830.3$ & $18: 38: 49.17$ & $-26: 28: 30.3$ & 7.803 & -9.174 & 14.032 & 0.586342 & 0.26 & 8.4 \\
\hline $\mathrm{J} 183855.27-242143.9$ & $18: 38: 55.27$ & $-24: 21: 43.9$ & 9.735 & -8.270 & 15.509 & 0.484700 & 0.40 & 15.5 \\
\hline J183855.30-242519.5 & $18: 38: 55.30$ & $-24: 25: 19.5$ & 9.681 & -8.296 & 14.526 & 0.563523 & 0.24 & 10.4 \\
\hline J183855.85-280435.5 & $18: 38: 55.85$ & $-28: 04: 35.5$ & 6.350 & -9.891 & 15.255 & 0.600842 & 0.32 & 15.4 \\
\hline J183857.43-280903.5 & $18: 38: 57.43$ & $-28: 09: 03.5$ & 6.28 & -9.9 & 14.339 & 0.625821 & & 10.1 \\
\hline J183857.75-280924.0 & $18: 38: 57.75$ & $-28: 09: 24.0$ & 6.280 & -9.932 & 13.690 & 0.634317 & 0.35 & 7.4 \\
\hline J183858.09-240456.9 & $18: 38: 58.09$ & $-24: 04: 56.9$ & 9.993 & -8.157 & 13.992 & 0.518285 & 0.32 & 7.7 \\
\hline $2.75-250127.3$ & $18: 3$ & $-25: 0$ & 9.146 & -8.586 & 14.383 & 0.57 & 0. & 9.9 \\
\hline J183907.71-271801.4 & 18:39:07.71 & $-27: 18: 01.4$ & 7.080 & -9.595 & 14.875 & 0.578339 & 0.28 & 12.5 \\
\hline J183908.74-261217.2 & 18:39:08.74 & $-26: 12: 17.2$ & 8.082 & -9.122 & 14.584 & 0.521360 & 0.31 & 10.3 \\
\hline J183910.27-261427.4 & $18: 39: 10.27$ & $-26: 14: 27.4$ & 8.051 & -9.143 & 13.333 & 0.658384 & 0.27 & 6.4 \\
\hline J183911.30-250716.6 & 18:39:11.30 & $-25: 07: 16.6$ & 9.072 & -8.658 & 13.083 & 0.573984 & 0.30 & 5.2 \\
\hline J183911.70-261839.8 & 18:39:11.70 & $-26: 18: 39.8$ & 7.990 & -9.179 & 14.754 & 0.550097 & 0.25 & 11.5 \\
\hline J183911.71-261839.9 & 18:39:11.71 & $-26: 18: 39.9$ & 7.990 & -9.179 & 14.766 & 0.550152 & 0.27 & 11.6 \\
\hline J183925.64-263539.0 & $18: 39: 25.64$ & $-26: 35: 39.0$ & 7.754 & -9.349 & 13.627 & 0.645332 & 0.31 & 7.3 \\
\hline J183934.41-243912.9 & $18: 39: 34.41$ & $-24: 39: 12.9$ & 9.536 & -8.531 & 15.989 & 0.515841 & 0.34 & 20.2 \\
\hline J183936.92-270303.2 & $18: 39: 36.92$ & $-27: 03: 03.2$ & 7.355 & -9.585 & 13.840 & 0.531604 & 0.33 & 7.3 \\
\hline J183937.15-264757.8 & $18: 39: 37.15$ & $-26: 47: 57.8$ & 7.586 & -9.476 & 15.110 & 0.483211 & 0.2 & 12.7 \\
\hline $\mathrm{J} 183940.84-250520.2$ & $18: 39: 40.84$ & $-25: 05: 20.2$ & 9.151 & -8.744 & 13.968 & 0.720088 & 0. & 9.1 \\
\hline J183940.98-235940.2 & $18: 39: 40.98$ & $-23: 59: 40.2$ & 10.146 & -8.264 & 13.594 & 0.450388 & 0.23 & 5.9 \\
\hline J183945.14-243437.9 & $18: 39: 45.14$ & $-24: 34: 37.9$ & 9.624 & -8.534 & 14.071 & 0.577207 & 0.28 & 8.5 \\
\hline $\mathrm{J} 183947.11-262523.7$ & $18: 39: 47.11$ & $-26: 25: 23.7$ & 7.945 & -9.347 & 15.599 & 0.543449 & 0.35 & 17.2 \\
\hline J183947.73-254231.6 & $18: 39: 47.73$ & $-25: 42: 31.6$ & 8.598 & -9.038 & 15.217 & 0.514846 & 0.27 & 13.9 \\
\hline J183953.19-240313.8 & $18: 39: 53.19$ & $-24: 03: 13.8$ & 10.113 & -8.332 & 15.142 & 0.563688 & 0.32 & 14.1 \\
\hline J183954.51-251028.9 & $18: 39: 54.51$ & $-25: 10: 28.9$ & 9.096 & -8.828 & 13.933 & 0.794553 & 0.22 & 9.4 \\
\hline J183955.80-243958.9 & 18:39:55.80 & $-24: 39: 58.9$ & 9.560 & -8.610 & 13.104 & 0.624103 & 0.36 & 5.5 \\
\hline J184001.55-265607.3 & 18:40:01.55 & $-26: 56: 07.3$ & 7.501 & -9.617 & 14.148 & 0.539843 & 0.30 & 8.5 \\
\hline J184003.37-273620.4 & 18:40:03.37 & $-27: 36: 20.4$ & 6.891 & -9.913 & 14.205 & 0.506937 & 0.32 & 8.4 \\
\hline J184004.91-282649.8 & 18:40:04.91 & $-28: 26: 49.8$ & 6.121 & -10.279 & 14.219 & 0.490504 & 0.34 & 8.3 \\
\hline J184009.98-275459.7 & $18: 40: 09.98$ & $-27: 54: 59.7$ & 6.616 & -10.068 & 14.502 & 0.724475 & 0.29 & 11.8 \\
\hline $\mathrm{J} 184022.02-261246.8$ & $18: 40: 22.02$ & $-26: 12: 46.8$ & 8.195 & -9.373 & 14.753 & 0.601931 & 0.27 & 12.1 \\
\hline J184022.63-273535.6 & $18: 40: 22.63$ & $-27: 35: 35.6$ & & -9.9 & & & & 14.3 \\
\hline $\mathrm{J} 184031.05-233527.1$ & $18: 40: 31.05$ & $-23: 35: 27.1$ & 10.597 & -8.258 & 14.143 & 0.601927 & 0.20 & 9.0 \\
\hline $\mathrm{J} 184036.15-240501.7$ & $18: 40: 36.15$ & $-24: 05: 01.7$ & 10.158 & -8.492 & 13.936 & 0.582632 & 0.24 & 8.0 \\
\hline J184055.96-272625.5 & $18: 40: 55.96$ & $-27: 26: 25.5$ & 7.127 & -10.017 & 14.093 & 0.585964 & 0.26 & 8.6 \\
\hline $\mathrm{J} 184058.67-264439.7$ & $18: 40: 58.67$ & $-26: 44: 39.7$ & 7.769 & -9.726 & 14.684 & 0.590528 & 0.36 & 11.6 \\
\hline J184058.91-272129.5 & $18: 40: 58.91$ & $-27: 21: 29.5$ & 7.207 & -9.991 & 14.830 & 0.554069 & 0.36 & 12.0 \\
\hline $\mathrm{J} 184100.13-242427.8$ & $18: 41: 00.13$ & $-24: 24: 27.8$ & 9.904 & -8.716 & 14.259 & 0.552175 & 0.31 & 9.1 \\
\hline J184100.66-245640.7 & $18: 41: 00.66$ & $-24: 56: 40.7$ & 9.416 & -8.952 & 14.853 & 0.591642 & 0.22 & 12.5 \\
\hline $\mathrm{J} 184100.87-265144.8$ & $18: 41: 00.87$ & $-26: 51: 44.8$ & 7.664 & -9.784 & 14.938 & 0.594166 & 0.33 & 13.1 \\
\hline J184101.61-262339.9 & $18: 41: 01.61$ & $-26: 23: 39.9$ & 8.094 & -9.585 & 15.301 & 0.714771 & 0.25 & 17.3 \\
\hline J184101.73-260135.9 & $18: 41: 01.73$ & $-26: 01: 35.9$ & 8.430 & -9.426 & 14.028 & 0.682170 & 0.23 & 9.1 \\
\hline J184101.77-260630.8 & $18: 41: 01.77$ & $-26: 06: 30.8$ & 8.355 & -9.461 & 14.489 & 0.607124 & 0.32 & 10.7 \\
\hline
\end{tabular}


Table A.1. continued.

\begin{tabular}{|c|c|c|c|c|c|c|c|c|}
\hline VVV ID & $\begin{array}{l}\text { RA (J2000.0) } \\
\text { hh:mm:ss.ss }\end{array}$ & $\begin{array}{c}\text { Dec (J2000.0) } \\
\text { dd:mm:ss.s }\end{array}$ & $\begin{array}{c}\ell \\
(\mathrm{deg})\end{array}$ & $\begin{array}{c}b \\
(\mathrm{deg})\end{array}$ & $\begin{array}{c}\left\langle K_{\mathrm{s}}\right\rangle \\
(\mathrm{mag})\end{array}$ & $\begin{array}{c}P \\
\text { (days) }\end{array}$ & $\begin{array}{l}\text { Amplitude } \\
\text { (mag) }\end{array}$ & $\begin{array}{c}d \\
(\mathrm{kpc})\end{array}$ \\
\hline $\mathrm{J} 184102.15-265412.7$ & $18: 41: 02.15$ & $-26: 54: 12.7$ & 7.629 & -9.806 & 14.674 & 0.513695 & 0.31 & 10.7 \\
\hline J184125.74-261120.4 & $18: 41: 25.74$ & $-26: 11: 20.4$ & 8.321 & -9.577 & 14.278 & 0.568870 & 0.27 & 9.3 \\
\hline $\mathrm{J} 184138.49-245701.6$ & $18: 41: 38.49$ & $-24: 57: 01.6$ & 9.473 & -9.083 & 14.317 & 0.572793 & 0.26 & 9.5 \\
\hline $\mathrm{J} 184142.59-244830.3$ & $18: 41: 42.59$ & $-24: 48: 30.3$ & 9.610 & -9.035 & 14.385 & 0.576399 & 0.26 & 9.9 \\
\hline J184147.89-254048.9 & $18: 41: 47.89$ & $-25: 40: 48.9$ & 8.823 & -9.432 & 13.910 & 0.691290 & 0.27 & 8.6 \\
\hline J184149.39-251819.0 & $18: 41: 49.39$ & $-25: 18: 19.0$ & 9.168 & -9.274 & 13.812 & 0.642426 & 0.25 & 7.9 \\
\hline $\mathrm{J} 184155.80-250922.8$ & $18: 41: 55.80$ & $-25: 09: 22.8$ & 9.314 & -9.231 & 13.503 & 0.564953 & 0.32 & 6.4 \\
\hline $\mathrm{J} 184201.50-264955.2$ & $18: 42: 01.50$ & $-26: 49: 55.2$ & 7.791 & -9.975 & 14.528 & 0.605012 & 0.20 & 10.9 \\
\hline J184205.46-265325.6 & $18: 42: 05.46$ & $-26: 53: 25.6$ & 7.744 & -10.013 & 14.273 & 0.632239 & 0.24 & 9.8 \\
\hline $\mathrm{J} 184214.98-244428.8$ & $18: 42: 14.98$ & $-24: 44: 28.8$ & 9.725 & -9.117 & 14.284 & 0.651347 & 0.32 & 10.0 \\
\hline $\mathrm{J} 184214.98-244428.9$ & $18: 42: 14.98$ & $-24: 44: 28.9$ & 9.725 & -9.117 & 14.281 & 0.651360 & 0.29 & 10.0 \\
\hline J184219.71-251756.3 & $18: 42: 19.71$ & $-25: 17: 56.3$ & 9.224 & -9.375 & 14.076 & 0.579818 & 0.26 & 8.5 \\
\hline J184219.71-251756.5 & $18: 42: 19.71$ & $-25: 17: 56.5$ & 9.224 & -9.375 & 14.087 & 0.579821 & 0.21 & 8.6 \\
\hline $\mathrm{J} 184231.55-244354.5$ & $18: 42: 31.55$ & $-24: 43: 54.5$ & 9.761 & -9.169 & 14.640 & 0.550712 & 0.27 & 10.9 \\
\hline J184234.04-243047.4 & $18: 42: 34.04$ & $-24: 30: 47.4$ & 9.965 & -9.082 & 14.599 & 0.528111 & 0.32 & 10.4 \\
\hline J184236.62-241630.9 & $18: 42: 36.62$ & $-24: 16: 30.9$ & 10.186 & -8.988 & 13.948 & 0.516198 & 0.24 & 7.5 \\
\hline $\mathrm{J} 184238.29-245140.4$ & $18: 42: 38.29$ & $-24: 51: 40.4$ & 9.654 & -9.248 & 16.130 & 0.569278 & 0.31 & 22.8 \\
\hline J184238.84-262334.5 & $18: 42: 38.84$ & $-26: 23: 34.5$ & 8.254 & -9.911 & 14.775 & 0.499758 & 0.27 & 11.0 \\
\hline $\mathrm{J} 184242.32-244715.0$ & $18: 42: 42.32$ & $-24: 47: 15.0$ & 9.728 & -9.230 & 14.167 & 0.650280 & 0.27 & 9.5 \\
\hline J184247.34-264254.4 & $18: 42: 47.34$ & $-26: 42: 54.4$ & 7.972 & -10.078 & 14.253 & 0.919261 & 0.20 & 11.9 \\
\hline $\mathrm{J} 184247.49-244122.0$ & $18: 42: 47.49$ & $-24: 41: 22.0$ & 9.826 & -9.205 & 14.181 & 0.634675 & & 9.4 \\
\hline $\mathrm{J} 184257.69-263500.0$ & $18: 42: 57.69$ & $-26: 35: 00.0$ & 8.110 & -10.057 & 15.815 & 0.606612 & & 20.3 \\
\hline J184257.83-263728.6 & $18: 42: 57.83$ & $-26: 37: 28.6$ & 8.072 & -10.075 & 13.713 & 0.516710 & 0.32 & 6.7 \\
\hline $\mathrm{J} 184306.98-260422.5$ & $18: 43: 06.98$ & $-26: 04: 22.5$ & 8.593 & -9.869 & 13.777 & 0.763744 & 0.30 & 8.6 \\
\hline $\mathrm{J} 184310.26-242512.7$ & $3: 10.26$ & -24 & 10.110 & -9.166 & 13.534 & 0.575848 & 0.28 & 6.5 \\
\hline $\mathrm{J} 184311.98-242224.3$ & $18: 43: 11.98$ & $2: 24.3$ & 10.155 & -9.151 & 15.097 & 0.583867 & 0.27 & 14.0 \\
\hline J184311.98-242224.4 & $18: 43: 11.98$ & $-24: 22: 24.4$ & 10.155 & -9.151 & 15.073 & 0.583870 & 0.28 & 13.9 \\
\hline J184317.97-245445.5 & $18: 43: 17.97$ & $-24: 54: 45.5$ & 9.673 & -9.4 & 13.907 & 0.702578 & 0.30 & 8.7 \\
\hline J184319.31-241434.0 & $18: 43: 19.31$ & $-24: 14: 34.0$ & 10.287 & -9.120 & 13.671 & 0.593867 & 0.24 & 7.1 \\
\hline J184322.05-253505.1 & $18: 43: 22.05$ & $-25: 35: 05.1$ & 9.065 & -9.710 & 14.495 & 0.825893 & 0.28 & 12.6 \\
\hline J184341.60-253310.2 & $18: 43: 41.60$ & $-25: 33: 10.2$ & 9.126 & -9.762 & 13.716 & 0.797703 & 0.30 & 8.5 \\
\hline $\mathrm{J} 184342.80-263056.3$ & $18: 43: 42.80$ & $-26: 30: 56.3$ & 8.245 & -10.18 & 14.771 & 0.434228 & 0.32 & 10.2 \\
\hline $\mathrm{J} 184345.08-244416.5$ & $18: 43: 45.08$ & $-24: 44: 16.5$ & 9.878 & -9.422 & 13.743 & 0.607489 & 0.31 & 7.4 \\
\hline $\mathrm{J} 184345.36-241857.9$ & $18: 43: 45.36$ & $-24: 18: 57.9$ & 10.264 & -9.241 & 14.302 & 0.617113 & 0.24 & 9.8 \\
\hline $\mathrm{J} 184356.24-250258.5$ & $18: 43: 56.24$ & $-25: 02: 58.5$ & 9.611 & -9.595 & 14.132 & 0.502434 & & 8.1 \\
\hline $\mathrm{J} 184402.41-263508.0$ & 18:44:02.41 & $-26: 35: 08.0$ & 8.213 & -10.276 & 14.227 & 0.500238 & & 8.5 \\
\hline $\mathrm{J} 184402.44-263049.2$ & $18: 44: 02.44$ & $-26: 30: 49.2$ & 8.279 & -10.245 & 14.569 & 0.49 & 0.27 & 9.9 \\
\hline $\mathrm{J} 184408.15-250322.6$ & $18: 44: 08.15$ & $-25: 03: 22.6$ & 9.625 & -9.638 & 14.245 & 0.551213 & 0.31 & 9.0 \\
\hline $\mathrm{J} 184412.37-261630.4$ & $18: 44: 12.37$ & $-26: 16: 30.4$ & 8.514 & -10.176 & 13.927 & 0.509583 & 0.33 & 7.4 \\
\hline $\mathrm{J} 184425.88-244355.6$ & $18: 44: 25.88$ & $-24: 43: 55.6$ & 9.951 & -9.559 & 14.707 & 0.543266 & 0.32 & 11.2 \\
\hline $\mathrm{J} 184438.62-254017.8$ & $18: 44: 38.62$ & $-25: 40: 17.8$ & 9.111 & -10.007 & 15.252 & 0.440556 & 0.37 & 13.0 \\
\hline $\mathrm{J} 184450.77-235622.9$ & 18:44:50.77 & $-23: 56: 22.9$ & 10.716 & -9.302 & 16.244 & 0.701346 & 0.30 & 27.0 \\
\hline $\mathrm{J} 184451.05-245604.8$ & $18: 44: 51.05$ & $-24: 56: 04.8$ & 9.807 & -9.732 & 14.340 & 0.767607 & 0.24 & 11.3 \\
\hline $\mathrm{J} 184506.49-243253.3$ & $18: 45: 06.49$ & $-24: 32: 53.3$ & 10.186 & -9.618 & 14.450 & 0.440176 & 0.36 & 8.8 \\
\hline J184507.09-252959.1 & 18:45:07.09 & $-25: 29: 59.1$ & 9.315 & -10.03 & 14.390 & 0.403729 & 0.35 & 8.2 \\
\hline J184508.79-251748.4 & $18: 45: 08.79$ & $-25: 17: 48.4$ & 9.504 & -9.948 & 14.203 & 0.560708 & 0.27 & 8.9 \\
\hline $\mathrm{J} 184511.06-235916.9$ & $18: 45: 11.06$ & $-23: 59: 16.9$ & 10.706 & -9.392 & 12.096 & 0.524332 & 0.30 & 3.1 \\
\hline $\mathrm{J} 184518.06-255731.3$ & $18: 45: 18.06$ & $-25: 57: 31.3$ & 8.912 & -10.263 & 13.566 & 0.591930 & 0.29 & 6.7 \\
\hline J184523.37-253935.3 & $18: 45: 23.37$ & $-25: 39: 35.3$ & & & & & & 9.8 \\
\hline $\mathrm{J} 184528.73-251435.5$ & $18: 45: 28.73$ & $-25: 14: 35.5$ & 9.586 & -9.993 & 13.903 & 0.480314 & & 7.1 \\
\hline $\mathrm{J} 184550.48-243009.2$ & $18: 45: 50.48$ & $-24: 30: 09.2$ & 10.301 & -9.749 & 13.678 & 0.556034 & 0.28 & 6.9 \\
\hline $\mathrm{J} 184614.16-251619.1$ & $18: 46: 14.16$ & $-25: 16: 19.1$ & 9.634 & -10.160 & 15.908 & 0.673292 & 0.34 & 22.4 \\
\hline $\mathrm{J} 184624.70-242411.0$ & $18: 46: 24.70$ & $-24: 24: 11.0$ & 10.449 & -9.824 & 14.299 & 0.526457 & 0.23 & 9.0 \\
\hline $\mathrm{J} 184634.15-250138.2$ & $18: 46: 34.15$ & $-25: 01: 38.2$ & 9.892 & -10.124 & 14.463 & 0.485661 & 0.32 & 9.3 \\
\hline J184636.94-250436.4 & $18: 46: 36.94$ & $-25: 04: 36.4$ & 9.851 & -10.154 & 13.868 & 0.513152 & 0.31 & 7.2 \\
\hline J184640.86-241711.2 & $18: 46: 40.86$ & $-24: 17: 11.2$ & 10.582 & -9.829 & 13.674 & 0.583652 & 0.28 & 7.0 \\
\hline $\mathrm{J} 184647.80-250704.2$ & $18: 46: 47.80$ & $-25: 07: 04.2$ & 9.831 & -10.209 & 14.806 & 0.630227 & 0.31 & 12.7 \\
\hline J184659.80-241551.8 & $18: 46: 59.80$ & $-24: 15: 51.8$ & 10.634 & -9.885 & 16.035 & 0.543925 & 0.24 & 21.2 \\
\hline $\mathrm{J} 184727.28-242721.0$ & $18: 47: 27.28$ & $-24: 27: 21.0$ & 10.504 & -10.061 & 14.548 & 0.717916 & 0.27 & 12.0 \\
\hline
\end{tabular}


F. Gran et al.: Mapping the outer bulge with RRab stars from the VVV Survey

Table A.2. 207 VVV RRab matching a variable in the General Cataloge of Variable Stars (GCVS).

\begin{tabular}{|c|c|c|c|c|c|}
\hline VVV ID & GCVS name & I VVV ID & GCVS name & I VVV ID & GCVS name \\
\hline | J2701020.22-411613.9 & V0462 CrA & | J2741313.37-375727.9 & IP CrA & J2755443.71-341148.1 & V2539 Sgr \\
\hline | J2701149.18-420358.3 & V0463 CrA & | J2741423.44-381809.1 & V0536 CrA & | J2755521.67-312549.7 & V3212 Sgr \\
\hline | J2702437.84-395238.8 & LM CrA & | J2741507.38-341727.9 & V2874 Sgr & | J2755625.55-315420.3 & V3216 Sgr \\
\hline | J2703434.23-413322.1 & V0469 CrA & | J2741640.08-341613.8 & V2878 Sgr & $\mathrm{J} 2755732.00-335639.4$ & V3213 Sgr \\
\hline | J2704032.86-393612.4 & LT CrA & | J2742121.33-334742.4 & V2896 Sgr & J2755903.99-320723.9 & V2540 Sgr \\
\hline | J2705536.91-392615.5 & MV CrA & | J2742130.69-361312.1 & V2889 Sgr & | J2760118.99-313547.9 & V3232 Sgr \\
\hline | J2705759.29-390948.3 & MY CrA & | J2742153.30-354403.3 & V2890 Sgr & | J2760406.80-325426.8 & V3242 Sgr \\
\hline | J2710402.86-390041.6 & NR CrA & | J2742329.54-353109.9 & V2899 Sgr & | J2760436.45-343055.4 & V3237 Sgr \\
\hline | J2710759.32-411700.5 & V0482 CrA & | J2742533.40-344836.6 & V0715 Sgr & | J2760556.25-323912.0 & V3248 Sgr \\
\hline | J2712342.04-421647.8 & V0483 CrA & | J2742625.55-333214.2 & V2914 Sgr & J2760606.68-341657.6 & V3244 Sgr \\
\hline | J2712711.57-421314.0 & V0486 CrA & | J2742639.96-335654.5 & V2912 Sgr & | J2760655.55-315252.5 & V3253 Sgr \\
\hline | J2712900.58-394418.1 & OS CrA & | J2742728.37-335146.2 & V2920 Sgr & | J2761027.42-324524.7 & V3258 Sgr \\
\hline | J2713907.68-393543.8 & $\mathrm{OX} \mathrm{CrA}$ & | J2742743.43-360357.6 & V2910 Sgr & | J2761128.83-32 & V3243 Sgr \\
\hline $4040.42-410352.1$ & V0398 CrA & | J2742802.34-372 & IQ CrA & | J2761405 & V2541 Sgr \\
\hline $4403.10-385517.7$ & V0399 CrA & | J274 & $30 \mathrm{Sgr}$ & I J2761706 & V3287 Sgr \\
\hline | J2720006.03-374514.8 & GK CrA & | J274 & V2933 Sgr & | J2761732.22-3 & V3281 Sgr \\
\hline | J2720110.20-372205.5 & GO CrA & | J2743205.87-341844.2 & V2514 Sgr & | J2762038.45-333047.8 & V3292 Sgr \\
\hline | J2721040.55-385557.8 & PY CrA & | J2743253.79-350633.2 & V2938 Sgr & | J2762108.32-305632.3 & V3301 Sgr \\
\hline | J2721703.45-405758.1 & V0499 CrA & | J2743337.74-325645.4 & V2516 Sgr & | J2762158.12-333306.2 & V3298 Sgr \\
\hline | J2722023.79-404349.4 & QV CrA & | J2743417.14-361754.9 & V2941 Sgr & | J2762248.05-305030.6 & V3304 Sgr \\
\hline | J2722138.38-390932.1 & QX CrA & | J2743610.45-363713.1 & V2950 Sgr & | J2762300.12-300107.2 & V1606 Sgr \\
\hline | J2722725.85-364838.9 & V0636 Sgr & | J2743934.92-351351.3 & V2967 Sgr & | J2762312.42- & \\
\hline | J2724552.44-385054.5 & V0506 C & | J2744307.84-3441| & & & V3309 Sgr \\
\hline | J2724759.12-372125.4 & $\mathrm{HN} \mathrm{CrA}$ & | J2744421.33-330033.9 & V2990 & J J2762515.00 & V3310 Sgr \\
\hline | J2724855.95-362110.3 & V06 & | J2744713. & & | J276270 & $7 \mathrm{Sgr}$ \\
\hline $\mathrm{J} 2$ & & $\mathrm{~J} 27$ & & $\mathrm{~J} 2$ & Sgr \\
\hline 34.7 & & | J27 & & | J276 & Sgr \\
\hline 109.1 & & | J27 & & | J276 & $\mathrm{Sgr}$ \\
\hline | J2730922.26-350757.4 & V26 & $\mathrm{J} 27$ & & & $3 \mathrm{Sgr}$ \\
\hline | J2731008.91-364330.2 & V2637 Sgr & | J2744 & 004 Sgr & & $27 \mathrm{Sgr}$ \\
\hline | J2731133.29-352457.1 & V0666 Sgr & | J2745055 & $16 \mathrm{Sgr}$ & & $30 \mathrm{Sgr}$ \\
\hline | J2731501.17-362149.5 & V0667 Sgr & | J2745144.3 & $19 \mathrm{Sgr}$ & & $6 \mathrm{Sgr}$ \\
\hline | J2731638.55-354528.3 & V2663 Sgr & | J2750154.7 & $053 \mathrm{Sgr}$ & | J2764121. & V3344 Sgr \\
\hline | J2732239.38-354714.7 & V2686 Sgr & | J2750300.4 & V2523 Sgr & | J2764232. & V3346 Sgr \\
\hline | J2732624.49-375455.2 & $\mathrm{HU} \mathrm{CrA}$ & | J2750422.74-352722.1 & & I J2764936 & V1302 Sgr \\
\hline | J2732728.37-392931.4 & V0355 CrA & | J2750922.26-335458.0 & & | J2765058.13 & V3357 Sgr \\
\hline | J2732809.37-361430.8 & & | J2750941.96-341525.6 & & | J2765205.6 & $3 \mathrm{Sgr}$ \\
\hline $945.94-363846.1$ & V06 & $\mathrm{J} 27$ & & J276 & $0 \mathrm{Sgr}$ \\
\hline $\mathrm{J} 2$ & V3s & $\mathrm{J} 27$ & & J276 & Sgr \\
\hline $\mathrm{J} 2$ & $\mathrm{HX}$ & | J27 & & | J276 & $2 \mathrm{Sgr}$ \\
\hline | J2 & Sgr & $\mathbf{J} 27$ & & | J277 & $7 \mathrm{Sgr}$ \\
\hline $\mathrm{J} 27$ & $3 \mathrm{Sgr}$ & | J275 & & $\mathrm{J} 277$ & V2555 Sgr \\
\hline | J2733638.45-351449.5 & V2734 Sgr & | J2751751.21-314347.1 & V3109 Sgr & J2770710.9 & V2554 Sgr \\
\hline | J2733824.84-355455.0 & V2739 Sgr & | J2752229.17-321151.5 & $600 \mathrm{Sgr}$ & | J2770759.8 & V3399 Sgr \\
\hline | J2733840.20-345906.3 & V2744 Sgr & | J2752614.30-340940.9 & $859 \mathrm{Sgr}$ & | J2770948.40-322348.4 & V3402 Sgr \\
\hline | J2734127.19-352035.6 & V2753 Sgr & | J2752744.07-313614.3 & V1291 Sgr & | J2771305.28-323916.3 & V3410 Sgr \\
\hline | J2734129.53-362705.8 & V2748 Sgr & | J2752752.85-340554.6 & $140 \mathrm{Sgr}$ & | J2771320.04-312151.2 & V3414 Sgr \\
\hline | J2734436.09-351324.8 & V2758 Sgr & | J2752826.49-342410.7 & V3141 Sgr & | J2771327.30-325251.9 & V1611 Sgr \\
\hline | J2734637.38-345411.4 & V2770 Sgr & | J2753005.28-322139.1 & V3150 Sgr & | J2771618.16-312636.2 & V3419 Sgr \\
\hline | J2734659.51-364323.7 & V2767 S & | J2753217.00-340440.9 & & | J2772302.34 & V2564 Sgr \\
\hline & & & & & V2566 Sgr \\
\hline | J2734959.70-365402.8 & V0694 Sgr & | J2753305.16-34382 & & | J21/2830. & V2567 Sgr \\
\hline | J2735029.17-344201.2 & & | J27534 & & | J2773243. & V3456 Sgr \\
\hline | J2735217.92-360538.7 & V0695 Sgr & | J2753615.8 & & | J2773409.84-28 & V1620 Sgr \\
\hline | J2735345.71-344202.4 & V0696 Sgr & | J2753746.29-312237.5 & V3169 Sgr & | J2773624.61-305556.1 & V3468 Sgr \\
\hline | J2735602.11-372806.6 & $\mathrm{XX} \mathrm{CrA}$ & | J2753937.74-333105.5 & V3170 Sgr & J2773653.55-300103.9 & V1621 Sgr \\
\hline | J2735942.07-352754.8 & V2806 Sgr & | J2753946.75-311226.6 & V3173 Sgr & | J2774119.92-322237.8 & V2355 Sgr \\
\hline | J2740129.76-382259.4 & V0533 CrA & | J2754046.99-315352.7 & V3174 Sgr & | J2774210.91-310954.4 & V3477 Sgr \\
\hline | J2740343.90-364712.9 & V2821 Sgr & | J2754118.51-360331.8 & V3168 Sgr & | J2774605.04-321729.7 & V3484 Sgr \\
\hline | J2740423.85-365626.5 & V0700 Sgr & | J2754257.08-313412.0 & V3178 Sgr & | J2774758.36-314114.2 & V3489 Sgr \\
\hline
\end{tabular}

Notes. VVV IDs and the respective GCVS names are presented. 
Table A.2. continued.

\begin{tabular}{lllllll}
\hline \hline | VVV ID & GCVS name & | VVV ID & GCVS name & VVV ID & GCVS name \\
\hline | J2740455.20-353803.8 & V0702 Sgr & J2754533.05-335723.9 & V3180 Sgr & J2774809.03-314441.3 & V3490 Sgr \\
| J2740715.58-351629.9 & V0706 Sgr & J2754558.25-320110.0 & V3188 Sgr & J2775727.88-311535.4 & V2574 Sgr \\
| J2740722.74-352507.5 & V0707 Sgr & J2754619.69-343303.0 & V3183 Sgr & J2780805.50-310924.2 & V1625 Sgr \\
| J2740748.40-351726.8 & V0708 Sgr & J2754708.67-341138.0 & V3186 Sgr & J2780843.71-312018.5 & V2582 Sgr \\
| J2740804.45-334141.8 & V2844 Sgr & J2754826.49-341617.6 & V1295 Sgr & J2781001.76-313547.4 & V2583 Sgr \\
| J2741013.01-354614.0 & V2846 Sgr & J2754847.92-314745.3 & V3193 Sgr & J2781358.59-312612.9 & V3539 Sgr \\
| J2741048.75-340122.3 & V2852 Sgr & J2754919.80-325428.2 & V3191 Sgr & | J2791305.39-291045.1 & V2597 Sgr \\
| J2741123.21-333359.5 & V2859 Sgr & J2754928.83-311656.0 & V3197 Sgr & J2792632.46-281530.6 & V1201 Sgr \\
| J2741224.96-352017.7 & V2857 Sgr & J2755001.53-333533.8 & V3192 Sgr & | J2793223.91-274127.0 & V2374 Sgr \\
\hline
\end{tabular}

